\title{
THE TANGENTIAL CAUCHY-RIEMANN COMPLEX ON SPHERES(1)
}

\author{
BY
}

\section{G.B. FOLLAND}

ABSTRACT. This paper investigates the $\bar{\partial}_{b}$ complex of Kohn and Rossi on the unit sphere in complex $n$-space (considered as the boundary of the unit ball). The methods are Fourier-analytic, exploiting the fact that the unitary group $U(n)$ acts homogeneously on the complex. We decompose the spaces of sections into irreducible components under the action of $U(n)$ and compute the action of $\bar{\partial}_{b}$ on each irreducible piece. We then display the connection between the $\bar{\partial}_{b}$ complex and the Dolbeault complexes of certain line bundles on complex projective space. Precise global regularity theorems for $\bar{\partial}_{b}$ are proved, including a Sobolev-type estimate for norms related to $\bar{\partial}_{b}$. Finally, we solve the $\bar{\partial}$-Neumann problem on the unit ball and obtain a proof by explicit calculations of the noncoercive nature of this problem.

\section{INTRODUCTION}

The tangential Cauchy-Riemann complex, or $\bar{\partial}_{b}$ complex, is a complex of differential operators living on the boundary of a complex manifold which arises as follows. Let $M$ be a complex manifold of (complex) dimension $n$ with smooth boundary $b M$, embedded in a slightly larger open manifold $M^{\prime}$; we assume $b M$ is defined by the equation $R=0$ where $R$ is a $C^{\infty}$ real-valued function on a neighborhood of $b M$ with $R<0$ inside $M, R>0$ outside $M$, and $d R \neq 0$ on $b M$. Let $A^{i j}$ be the vector bundle of differential forms of type $(i, j)$ on $M^{\prime}$, and let $\underline{A}^{i j}$ be the sheaf of germs of sections of $A^{i j}$ over $M$ which are smooth up to the boundary, i.e. which are restrictions of smooth sections over $M^{\prime}$. Since $\bar{\partial} R \neq 0$ on $b M$, the set of all $\xi \in \bigoplus_{i j} A^{i j} \mid b M$ of the form $\xi=\theta \wedge \bar{\partial} R$ is a subbundle of $\bigoplus_{i j}{ }^{A^{i j}} \mid b M$ which we denote by $I(\bar{\partial} R)$ (for "the ideal generated by $\bar{\partial} R$ "). Let $B^{i j}$ be the quotient of $A^{i j} \mid b M$ by $\left(A^{i j} \mid b M\right) \cap I(\bar{\partial} R)$, and let $\underline{B}^{i j}$ be its sheaf of germs of sections. Then if $\underline{C}^{i j}$ denotes the subsheaf of $\underline{A}^{i j}$ consisting of germs of sections whose restriction to $b M$ lies in $I\left(\bar{\partial} R\right.$ ) (so in particular $\underline{C}^{i 0}$ consists of germs of sections of $A^{i 0}$ vanishing on $b M$ ), we have the exact sequence

Received by the editors October 21, 1971.

AMS 1970 subject classifications. Primary 35H05, 35N15; Secondary 33A40, 33A45, 43A75.

Key words and phrases. Tangential Cauchy-Riemann operators, subelliptic operators, transversally elliptic operators, representations of $U(n)$, spherical harmonics, Sobolev estimates, $\bar{\partial}$-Neumann problem, Bessel functions.

(1) This work was supported by a National Science Foundation Graduate Fellowship and a grant from the Alfred P. Sloan Foundation. 


$$
0 \longrightarrow \underline{C}^{i j} \rightarrow \underline{A}^{i j} \rightarrow \underline{B}^{i j} \rightarrow 0 \text {. }
$$

The sheaves $\underline{A}^{i j}$ form a complex via the Cauchy-Riemann operator $\bar{\partial}$ :

$$
0 \rightarrow \underline{A}^{i 0} \stackrel{\bar{\partial}}{\longrightarrow} \underline{A}^{i 1} \stackrel{\bar{\partial}}{\longrightarrow} \cdots \stackrel{\bar{\partial}}{\rightarrow} \underline{A}^{i n} \rightarrow 0 .
$$

Since $\bar{\partial}(u \wedge \bar{\partial} R)=\bar{\partial} u \wedge \bar{\partial} R, \bar{\partial}\left(\underline{C}^{i j}\right) \subset \underline{C}^{i(j+1)}$. Therefore, by the usual diagram chase, there is induced from (1) and (2) a complex

$$
0 \rightarrow \underline{B}^{i 0} \stackrel{\bar{\partial}_{b}}{\longrightarrow} \underline{B}^{i 1} \stackrel{\bar{\partial}_{b}}{\longrightarrow} \cdots \stackrel{\bar{\partial}_{b}}{\longrightarrow} \underline{B}^{i(n-1)} \rightarrow 0,
$$

the $\bar{\partial}_{b}$ complex. It is easily seen that the $\bar{\partial}_{b}$ complex is independent of the choice of $R$, up to isomorphism. Although the $\bar{\partial}$ complex is elliptic, the $\bar{\partial}_{b}$ complex is not; in fact, the cotangent vectors $i(\bar{\partial}-\partial) R$ are characteristic at each point.

We as sume from now on that $M^{\prime}$ carries a hermitian metric. We denote the pointwise scalar products with respect to this metric by pointing brackets $\langle$, and the global (integrated) scalar products by round brackets $($,$) ; thus (u, v)=$ $\int\langle u, v\rangle$. We further denote the $L^{2}$ norms with respect to $($,$) by \|\|$ and the Sobolev $s$-norms by \|\|$_{s}$.

$B^{i j}$ may now be identified with the (pointwise) orthogonal complement of $I(\bar{\partial} R)$ in $A^{i j} \mid b M$, and the operator $\bar{\partial}_{b}$ is defined as follows. If $u \in \Gamma\left(\underline{B}^{i j}\right)$ ( $\Gamma$ will always denote spaces of global sections), let $u^{\prime}$ extend $u$ smoothly to $M^{\prime}$. Then $\bar{\partial}_{b} u$ is the orthogonal projection of $\bar{\partial} u^{\prime} \mid b M$ onto $B^{i(j+1)}$, and this definition is independent of the extension $u^{\prime}$. We may also define the formal adjoint $\delta_{b}$ of $\bar{\partial}_{b}$ by $\left(\delta_{b} u, v\right)=\left(u, \bar{\partial}_{b} v\right)$, which yields the adjoint complex

$$
0 \leftarrow \underline{B}^{i 0} \stackrel{\mathfrak{D}_{b}}{\longleftarrow} \underline{B}^{i 1} \stackrel{\mathfrak{D}_{b}}{\longleftarrow} \ldots \stackrel{\mathrm{S}_{b}}{\longleftarrow} \underline{B}^{i(n-1)} \leftarrow 0
$$

and the Laplacian $\square_{b}=\bar{\partial}_{b} \delta_{b}+\delta_{b} \bar{\partial}_{b}$.

The tangential Cauchy-Riemann operators were studied by H. Lewy [10] in the case $n=2$ in connection with the problem of finding a holomorphic function in a region of $\mathbf{C}^{2}$ with given boundary values. This work was later extended by Kohn and Rossi [9], who formalized the notion of " $\bar{\partial}_{b}$ complex." Meanwhile, however, Lewy had been led by his work to the discovery of a smooth differential equation which is not locally solvable [11], which has had vast repercussions in the theory of partial differential equations (cf. Trèves [16]). In fact, Lewy's example is the $\bar{\partial}_{b}$ operator for a certain strongly pseudoconvex domain in $\mathbf{C}^{2}$, and it can be shown from Hörmander's criterion [16] that the $\bar{\partial}_{b}$ operator for any strongly pseudoconvex 2-manifold is not locally solvable.

If $M$ is compact, the $\bar{\partial}_{b}$ complex on $b M$ is intimately connected with the $\bar{\partial}$-Neumann problem on $M$. A form $u \in \Gamma\left(\underline{A}^{i j}\right)$ is said to satisfy the $\bar{\partial}$-Neumann 
boundary conditions if $u \mid b M \in \Gamma\left(\underline{B}^{i j}\right)$ and $\bar{\partial} u \mid b M \in \Gamma\left(\underline{B}^{i(j+1)}\right)$. A simple argument by Stokes' theorem shows that these conditions are equivalent to the requirement that $(u, \bar{\partial} v)=(b u, v)$ for all $v \in \Gamma\left(\underline{A}^{i(j-1)}\right)$ and $(\bar{\partial} u, \bar{\partial} v)=(\bar{b} \bar{\partial} u, v)$ for all $v \in \Gamma\left(\underline{A}^{i j}\right)$, where $\delta$ is the formal adjoint of $\bar{\partial}$. If we form the Laplacian $\square=$ $\bar{\partial} \delta+\delta \overline{\bar{\partial}}$, the $\bar{\partial}$-Neumann problem can be roughly stated as the problem of solving the equation $\square u=v$ where $u$ satisfies the $\bar{\partial}$-Neumann conditions. More precisely, the restriction of $\square$ to the space of forms satisfying the $\bar{\partial}$-Neumann conditions is a positive hermitian operator which has a natural extension (the Friedrichs extension) to a selfadjoint operator; the $\bar{\partial}$-Neumann problem is the analysis of this operator.

The $\bar{\partial}$-Neumann problem is a noncoercive boundary value problem; that is, one does not have the estimate $\|u\|_{s+2}^{2} \leq c\left(\|\square u\|_{s}^{2}+\|u\|_{s}^{2}\right)$. However, Kohn [6] (cf. also Kohn and Nirenberg [8]) has shown that under suitable pseudoconvexity conditions, the estimate $\|u\|_{s+1}^{2} \leq c\left(\|\square u\|_{s}^{2}+\|u\|_{s}^{2}\right)$ holds, and that in this case one obtains existence and regularity up to the boundary, hypo-ellipticity, finite-dimensionality of the harmonic space, and other nice properties. In particular, this leads to a Hodge decomposition for the $\bar{\partial}$ complex, which has important applications to the theory of several complex variables.

The $\bar{\partial}_{b}$ complex is the boundary complex associated to the $\bar{\partial}$-Neumann problem in accordance with Spencer's general theory of Neumann problems for overdetermined elliptic systems (cf. Sweeney [15], also Kohn and Rossi [9]).

The $\bar{\partial}_{b}$ complex is important for another reason. Under suitable pseudoconvexity conditions, the Laplacian $\square_{b}$ has been shown by Kohn [7] to satisfy the "1/2-estimate" $\|u\|_{1 / 2}^{2} \leq c\left(\left(\square_{b} u, u\right)+\|u\|^{2}\right)$. Operators satisfying such "subelliptic" estimates have many of the qualitative properties of elliptic operators, such as regularity of weak solutions and compactness of the Green's operator (cf. Kohn and Nirenber $g$ [8]), and they have recently attracted considerable attention from Hörmander, Egorov, and others. $\square_{b}$ is the best-known example of the se operators and is thus a good starting point for work in this area.

It is our purpose here to investigate in detail the case $M=B_{n}=$ $\left\{z \in \mathbb{C}^{n}:|z| \leq 1\right\}, b M=S_{n}=\left\{z \in \mathbb{C}^{n}:|z|=1\right\}$. In this situation the $\bar{\partial}_{b}$ complex has an added significance: it is the prototype example of the "transversally elliptic" operators currently being studied by Atiyah and his coworkers. An operator on the manifold $X$ is said to be transversally elliptic with respect to a group action on $X$ if it commutes with the action, and the cotangent vectors orthogonal to the orbits of the group are noncharacteristic. In our case, the circle group $S_{1}$ acts (as a subset of $\mathrm{C}$ ) by scalar multiplication on $S_{n}$, and the characteristics are precisely the cotangent vectors to the orbits of this action.

We shall restrict our attention to forms of purely antiholomorphic type, i.e. 
$i=0$. All the essential features of the situation are present in this special case, so we will lose no interesting information while gaining considerably in notational simplicity-a boon for which the reader will soon have cause to be grateful. In any event, it will be clear how to modify our procedure to obtain analogous results for $i>0$.

Let us take a closer look at the operator $\bar{\partial}_{b}$ on $S_{n}$. We take $R=r-1$, where $r=\left(\Sigma_{1}^{n} z_{a} \bar{z}_{a}\right)^{1 / 2}$ is the distance from the origin. Let $f \in \Gamma\left(\underline{B}^{00}\right)$ be a function on $S_{n}$, and let $f^{\prime}$ extend $f$ to $\mathbf{C}^{n}$. Then $\bar{\partial}_{b} f$ is the restriction to $S_{n}$ of

$$
\begin{aligned}
\bar{\partial} f^{\prime} & -\left(\left\langle\bar{\partial} f^{\prime}, \overline{\partial r}\right\rangle /\langle\bar{\partial} r, \overline{\partial r}\rangle\right) \overline{\partial r} \\
= & \sum_{a=1}^{n} \frac{\partial f^{\prime}}{\partial \bar{z}_{a}} d \bar{z}_{a}-2\left\langle\sum_{b=1}^{n} \frac{\partial f^{\prime}}{\partial \bar{z}_{b}} d \bar{z}_{b}, \frac{1}{2 r} \sum_{b=1}^{n} z_{b} d \bar{z}_{b}\right\rangle \frac{1}{2 r} \sum_{a=1}^{n} z_{a} d \bar{z}_{a} \\
= & \sum_{a=1}^{n}\left(\frac{\partial f^{\prime}}{\partial \bar{z}_{a}}-\frac{z_{a}}{r^{2}} \sum_{b=1}^{n} \bar{z}_{b} \frac{\partial f^{\prime}}{\partial \bar{z}_{b}}\right) d \bar{z}_{a}
\end{aligned}
$$

since $\left\langle d \bar{z}_{a}, d \bar{z}_{b}\right\rangle=2 \delta_{a b}$. When we restrict to $S_{n}$, of course, we may neglect the factor of $r^{2}$.

In particular,

$$
\bar{\partial}_{b} \bar{z}_{i}=d \bar{z}_{i}-\bar{z}_{i} \sum_{a=1}^{n} z_{a} d \bar{z}_{a}=d \bar{z}_{i}-2 \bar{z}_{i} \bar{\partial}_{r}
$$

The forms $\bar{\partial}_{b} \bar{z}_{i}$ will be of fundamental importance, and we denote them by $\zeta_{i}$. Since $\left\{d \bar{z}_{i_{1}} \wedge \ldots \wedge d \bar{z}_{i_{j}}: i_{1}<\cdots<i_{j}\right\}$ is a basis for $A^{0 j}$ at each point, $\left\{\zeta_{i_{1}} \wedge \ldots \wedge \zeta_{i_{j}}: i_{1}<\cdots<i_{j}\right\}$ spans $B^{0 j}$ at each point. It is not a basis, of course: the $\zeta_{i}^{\prime}$ 's satisfy $\Sigma_{1}^{n} z_{i} \zeta_{i}=0$.

We can now write down the general formula for $\bar{\partial}_{b}$. For functions, with $f, f^{\prime}$ as above,

$$
\begin{aligned}
\bar{\partial}_{b} f & =\sum_{i} \frac{\partial f^{\prime}}{\partial \bar{z}_{i}} d \bar{z}_{i}-\sum_{i, a} z_{i} \bar{z}_{a} \frac{\partial f}{\partial \bar{z}_{a}} d \bar{z}_{i} \\
& =\sum_{i} \frac{\partial f}{\partial \bar{z}_{i}}-\sum_{i, a} z_{a} \bar{z}_{i} \frac{\partial f^{\prime}}{\partial \bar{z}_{i}} d \bar{z}_{a} \\
& =\sum_{i} \frac{\partial f^{\prime}}{\partial \bar{z}_{i}}\left(d \bar{z}_{i}-\bar{z}_{i} \sum_{a} z_{a} d \bar{z}_{a}\right)=\sum_{i} \frac{\partial f}{\partial \bar{z}_{i}} \zeta_{i} .
\end{aligned}
$$

$\bar{\partial}_{b}$ now extends as a derivation in the usual way: 


$$
\begin{aligned}
\bar{\partial}_{b}\left(\sum_{i_{1}<\cdots<i_{j}} f_{i_{1} \cdots i_{j}} \zeta_{i_{1}} \wedge \cdots \wedge \zeta_{i_{j}}\right) \\
=\sum_{i_{1}<\cdots<i_{j}} \bar{\partial}_{b} f_{i_{1} \cdots i_{j}} \wedge \zeta_{i_{1}} \wedge \cdots \wedge \zeta_{i_{j}} \\
=\sum_{i_{1}<\cdots<i_{j}} \sum_{a} \frac{\partial f_{i_{1} \cdots i_{j}}^{\prime}}{\partial \bar{z}_{a}} \zeta_{a} \wedge \zeta_{i_{1}} \wedge \cdots \wedge \zeta_{i_{j}}
\end{aligned}
$$

where $f_{i_{1} \cdots i_{j}}^{\prime}$ extends $f_{i_{1} \cdots i_{j}}$.

Our program will be to make a detailed study of the $\bar{\partial}_{b}$ complex by exploiting its symmetry with respect to the unitary group $U(n)$. The sphere $S_{n}$ is a homogeneous space, $S_{n} \cong U(n) / U(n-1)$, and all the vector bundles $B^{0 j}$ are homogeneous bundles since $U(n)$ commutes with $\bar{\partial}$ and preserves the radial function $r$. For the same reason, $U(n)$ commutes with $\bar{\partial}_{b}$. We will therefore obtain information about $\bar{\partial}_{b}$ by decomposing the spaces of sections under the group action. This decomposition is accomplished and a formula is obtained for the eigenvalues of the $\bar{\partial}_{b}$ complex in Chapter II. In Chapter III we show how the decomposition under the circle action relates the $\bar{\partial}_{b}$ complex to the Dolbeault complexes of line bundles over $C P^{n-1}$. We then derive in Chapter IV the global regularity results for $\bar{\partial}_{b}$ in a more precise form than can be obtained from the general estimates, and we prove a Sobolev-type theorem for norms related to $\square_{b}$. Finally, in Chapter $\mathrm{V}$ we combine these methods with the theory of Bessel functions to solve the $\bar{\partial}$-Neumann problem on the ball $B_{n}$ by eigenform expansions and obtain a rather striking demonstration by explicit calculations of the noncoercive nature of this problem.

The author wishes to express his gratitude to J. J. Kohn, E. M. Stein, and D. C. Spencer for the ir invaluable advice and encouragement while this work was in preparation.

\section{GROUP REPRESENTATIONS (DECOMPOSITION OF THE $\bar{\partial}_{b}$ COMPLEX)}

1. Preliminaries. We shall assume the basic theory of unitary representations of compact groups as presented in Stein [14] or Weil [18]. In addition, we need the notion of induced representations. If $G$ is a compact Lie group and $H$ a closed subgroup, and $\rho$ is a unitary representation of $H$ on a Hilbert space $V$, we may form the homogeneous vector bundle $\widetilde{V}=V \times_{H} G$ on $G / H$ with fiber $V$; then there is a natural representation of $G$ on sections of $\widetilde{V}$ given by $(g s)(x)=$ $g\left[s\left(g^{-1} x\right)\right]$. Let $\mu$ be a $G$-invariant measure on $G / H$ (which always exists for compact $G)$, and let $L^{2}(\tilde{V}, \mu)$ be the completion of $\Gamma(\tilde{V})$ with respect to the scalar product induced by $\mu$. Then the representation of $G$ on $\Gamma(\widetilde{V})$ extends to a 
unitary representation of $G$ on $L^{2}(\tilde{V}, \mu)$ which is called the induced representation of $\rho$ and is denoted by $i(\rho)$. The fundamental fact about induced representations is the following:

Frobenius Reciprocity Theorem. Let $G$ be a compact group, $H$ a closed subgroup, $\sigma$ an irreducible representation of $G$, and $\rho$ an irreducible representation of $H$. Then $\sigma$ occurs in $i(\rho)$ with the same multiplicity as $\rho$ occurs in $\sigma \mid H$.

Proof. See Weil $[18, \$ 23]$. (Note. Here, as in other places, we identify the notions of "representation" and "equivalence class of representations" par abus de langage.)

Now $S_{n} \cong U(n) / U(n-1)$ where we think of $U(n-1)$ as the isotropy group of the base point $z_{0}=(0,0, \cdots, 0,1)$, that is, we embed $U(n-1)$ in $U(n)$ by $A \mapsto\left(\begin{array}{cc}A & 0 \\ 0 & 1\end{array}\right)$, and we take as invariant measure the natural measure on $S_{n}$ induced from $\mathrm{C}^{n}$. Since $U(n)$ preserves the form $\overline{\partial r}$, the bundles $B^{0 j}$ are homogeneous bundles. Moreover, since $\left.2 \overline{\partial r}\right|_{z_{0}}=\left.d \bar{z}_{n}\right|_{z_{0}}$, the fiber $\left.B^{0 j}\right|_{z_{0}}$ is just the span of $\left\{\left.d \bar{z}_{i_{1}} \wedge \ldots \wedge d \bar{z}_{i_{j}}\right|_{z_{0}}: i_{1}<\cdots<i_{j} \leq n-1\right\}$. Therefore the representation of $U(n-1)$ on $B^{0 j} \mid z_{0}$ is just the $j$ th exterior power of the representation on the antiholomorphic covectors on $\mathrm{C}^{n-1}$. This representation, however, is the conjugate of the representation on the holomorphic covectors, which in turn is the contragradient of the standard representation on $\mathrm{C}^{n-1}$. Since contragradient is the same as conjugate for unitary representations, we have the following proposition:

Theorem 1. Let $\Re^{j}$ be the Hilbert space completion of $\Gamma\left(\underline{B}^{0 j}\right)$. Then the representation of $U(n)$ on $B^{j}$ is the induced representation of the jth exterior power of the standard representation of $U(n-1)$ on $\mathrm{C}^{n-1}$.

In order to analyze the spaces $\mathfrak{B}^{j}$, we need more specific information about representations of the unitary groups.

2. Representations of $U(n)$. We present here a brief outline of the representation theory of $U(n)$ as developed in Boerner [1] and Weyl [19]. For details and proofs the reader is referred to these treatises, especially to Boerner.

The irreducible representations of $U(n)$ are classified by $n$-tuples of integers $\left(m_{1}, \cdots, m_{n}\right)$ with $m_{1} \geq m_{2} \geq \cdots \geq m_{n}$; the representation corresponding to $\left(m_{1}, \cdots, m_{n}\right)$ will be denoted by $\rho\left(m_{1}, \cdots, m_{n}\right)$. These representations may be described in the following way.

First assume $m_{n} \geq 0$. We may then form the Young diagram of the $n$-tuple $\left(m_{1}, \cdots, m_{n}\right)$, which is a semirectangular array of boxes with $m_{1}$ boxes in the first row $m_{2}$ boxes in the second row, $\cdots, m_{i}$ boxes in the $i$ th row. For example, the Young diagram of $(6,5,3,1)$ is 


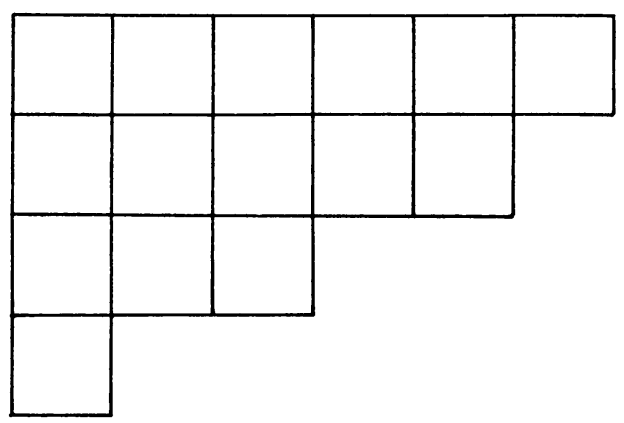

The standard tableau is obtained by putting the numbers from 1 to $\Sigma_{1}^{n} m_{i}$ in the boxes in order along the columns, for example

\begin{tabular}{|l|l|l|l|l|l|}
\hline 1 & 5 & 8 & 11 & 13 & 15 \\
\hline 2 & 6 & 9 & 12 & 14 & \multicolumn{2}{|}{} \\
\hline 3 & 7 & 10 & \multicolumn{2}{|l}{} \\
\hline 4 & \multicolumn{1}{|l}{} \\
\cline { 1 - 3 } & &
\end{tabular}

The standard tableau defines an element $T$ of the group ring of the symmetric group on $\Sigma m_{i}$ letters as follows. Let $\sigma$ denote any permutation of $\left(1, \cdots, \Sigma m_{i}\right)$ which leaves the rows of the tableau invariant, i.e. which interchanges only numbers occurring in the same row. Likewise, let $\tau$ denote any permutation which leaves the columns invariant. Then $T=\Sigma_{\tau} \Sigma_{\sigma}(\operatorname{sgn} \tau) \tau \sigma$. It can be shown that $T$ is idempotent up to a constant factor.

Now $U(n)$ acts on the tensor space $\otimes^{\Sigma m_{i}} \mathrm{C}^{n}$ by the $\Sigma_{m_{i}}$ th tensor power of the standard representation on $\mathrm{C}^{n}$. On the other hand, the symmetric group, and hence its group ring, acts on $\otimes^{\Sigma m_{i}} \mathrm{C}^{n}$ by permutation of indices. The element $T$ thus defines a projection (up to a constant factor) on $\otimes^{\Sigma m_{i}} C^{n}$ whose range $V\left(m_{1}, \cdots, m_{n}\right)$ is an irreducible invariant subspace under the action of $U(n)$. The representation of $U(n)$ on $V\left(m_{1}, \cdots, m_{n}\right)$ is $\rho\left(m_{1}, \cdots, m_{n}\right)$.

Several remarks are in order at this point. The effect of the "Young symmetrizer" $T$ on a tensor is first to symmetrize it with respect to the indices occurring in the same rows of the standard tableau, then to skew-symmetrize it with respect to the indices occuring in the same columns. (Note that the latter operation partially undoes the symmetry achieved by the former.) The space $V\left(m_{1}, \cdots, m_{n}\right)$ may therefore be regarded as a subspace of 


$$
\left[\otimes^{m} n\left(\bigwedge^{n} \mathrm{C}^{n}\right)\right] \otimes\left[\otimes^{m_{n-1}-m_{n}}\left(\bigwedge^{n-1} \mathrm{C}^{n}\right)\right] \otimes \ldots \otimes\left[\bigotimes^{m_{1-m^{2}}} \mathrm{C}^{n}\right]
$$

In particular, if $m_{1}=\cdots=m_{j}=1, m_{j+1}=\cdots=m_{n}=0, V\left(m_{1}, \cdots, m_{n}\right)=\bigwedge^{j} \mathrm{C}^{n}$. Hence the $j$ th exterior power of the standard representation on $\mathbf{C}^{n}$ is $\rho(1,1, \cdots, 1,0,0, \cdots, 0)$ ( $j$ ones).

It is now easy to see that

$$
\rho\left(m_{1}, \cdots, m_{n}\right)=(\text { det })^{m_{n}} \rho\left(m_{1}-m_{n}, \cdots, m_{n-1}-m_{n}, 0\right) .
$$

We therefore take this equation as the definition of $\rho\left(m_{1}, \cdots, m_{n}\right)$ in the general case. It can then be shown that $\left\{\rho\left(m_{1}, \cdots, m_{n}\right): m_{i} \in \mathbf{Z}, m_{1} \geq \cdots \geq m_{n}\right\}$ forms a complete set of inequivalent irreducible representations of $U(n)$.

The contragradient or conjugate representation to $\rho\left(m_{1}, \cdots, m_{n}\right)$ is $\rho\left(-m_{n}, \cdots,-m_{1}\right)$. In particular, the contragradient to $\rho(1,0, \cdots, 0)$ is $\rho(0, \cdots, 0,-1)=(\text { det })^{-1} \rho(1, \cdots, 1,0)$; the reader will recognize this fact as nothing more than the relation between the $(n-1) \times(n-1)$ minors of a matrix and its inverse transpose. Now consider a representation $\rho\left(m_{1}, \cdots, m_{n}\right)$ with $m_{n}<0, m_{n-1} \geq 0$. This may be regarded as acting on a subspace $V\left(m_{1}, \cdots, m_{n}\right)$ of

$$
\left[\otimes^{m_{n-1}-m_{n}}\left(\bigwedge^{n-1} \mathrm{C}^{n}\right)\right] \otimes \ldots \otimes\left[\otimes^{m_{1}-m_{2}} \mathrm{C}^{n}\right]
$$

via (det) ${ }^{m} n$ times the standard representation on this space. But by the preceding remarks, $\bigwedge^{n-1} \mathrm{C}^{n}$ with the action (det) ${ }^{-1} \rho(1, \cdots, 1,0)$ is isomorphic as a $U(n)$-module to $\mathbf{C}^{n}{ }^{*}$, the dual space of $\mathbf{C}^{n}$. Therefore we may regard $V\left(m_{1}, \cdots, m_{n}\right)$ as a subspace of

$$
\begin{aligned}
& {\left[\bigotimes^{-m_{n}} C^{n^{*}}\right] \otimes\left[\bigotimes^{m_{n-1}}\left(\wedge^{n-1} C^{n}\right)\right]}
\end{aligned}
$$

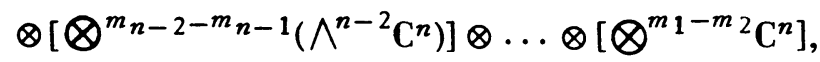

and the representation $\rho\left(m_{1}, \cdots, m_{n}\right)$ is given by the standard action of $U(n)$ on this space.

Let $e_{1}, \cdots, e_{n}$ be the canonical basis for $\mathbf{C}^{n}$. Then it is readily verified that for $m_{n} \geq 0$ the tensor

$$
\begin{aligned}
P( & \left.m_{1}, \cdots, m_{n}\right) \\
= & {\left[\bigotimes^{m_{n}}\left(e_{1} \wedge \ldots \wedge e_{n}\right)\right] } \\
& \otimes\left[\otimes^{m_{n-1}-m_{n}}\left(e_{1} \wedge \ldots \wedge e_{n-1}\right)\right] \otimes \ldots \otimes\left[\otimes^{m_{1}-m_{2}} e_{1}\right]
\end{aligned}
$$


lies in $V\left(m_{1}, \cdots, m_{n}\right)$. (One need only check that the Young symmetrizer leaves it fixed.) Likewise for $m_{n}<0, m_{m-1} \geq 0$, the tensor

$$
\begin{aligned}
& P\left(m_{1}, \cdots, m_{n}\right) \\
& \quad=\left[\otimes^{-m_{n}} e_{n}^{*}\right] \otimes\left[\otimes^{m_{n-1}}\left(e_{1} \wedge \ldots \wedge e_{n-1}\right)\right] \otimes \ldots \otimes\left[\otimes^{m_{1}-m_{2} e_{1}}\right]
\end{aligned}
$$

lies in $V\left(m_{1}, \cdots, m_{n}\right)$ since $e_{n}^{*}$ corresponds to $e_{1} \wedge \ldots \wedge e_{n-1}$ under the identification of $\mathrm{C}^{n^{*}}$ with $\bigwedge^{n-1} \mathrm{C}^{n} . P\left(m_{1}, \cdots, m_{n}\right)$ will be called the primitive vector for $\rho\left(m_{1}, \cdots, m_{n}\right)$. (This terminology comes from the theory of semisimple Lie algebras, which applies if we restrict our representations to the simple group $\operatorname{SU}(n)$.)

Finally, we need to know how $\rho\left(m_{1}, \cdots, m_{n}\right)$ decomposes when it is restricted to $U(n-1)$. This question is answered by the Branching Theorem:

$$
\rho\left(m_{1}, \cdots, m_{n}\right) \mid U(n-1)=\bigoplus_{m_{1} \geq \mu_{1} \geq m_{2} \geq \cdots \geq \mu_{n-1} \geq m_{n}} \rho\left(\mu_{1}, \cdots, \mu_{n-1}\right) .
$$

3. Decomposition of the spaces $\mathfrak{B}^{j}$. We are now ready to decompose the $\bar{\partial}_{b}$ complex under the action of $U(n)$. The first step is to identify the irreducible representations occurring in $\mathfrak{B}^{j}$.

We introduce the following notation: if $a, b, \cdots \in \mathbf{Z}$ and $k_{1}, k_{2}, \cdots \in \mathbf{Z}^{+}$, $\left(\underline{a}_{k_{1}}, \underline{b}_{k_{2}}, \cdots\right)$ denotes the $\left(k_{1}+k_{2}+\cdots\right)$-tuple whose first $k_{1}$ entries are $a$, whose next $k_{2}$ entries are $b$, etc. For example, $\left(\underline{1}_{3},-\underline{1}_{2},-\underline{2}_{2}\right)=$ $(1,1,1,-1,-1,-2,-2)$. Naturally we still write $a$ instead of $\underline{a}_{1}$, and $\underline{a}_{0}$ is a zerotuple, so $\left(\underline{a}_{0}, \underline{b}_{k}, \cdots\right)=\left(\underline{b}_{k}, \ldots\right)$.

\section{Theorem 2.}

$$
\mathcal{B}^{0} \cong \bigoplus_{p \geq 0, q \geq 0} \rho\left(q, \underline{0}_{n-2},-p\right) ; \quad B^{n-1} \cong \bigoplus_{p \geq-1, q \geq 1}^{\bigoplus} \rho\left(q, \underline{1}_{n-2},-p\right)
$$

and for $1 \leq j \leq n-2$,

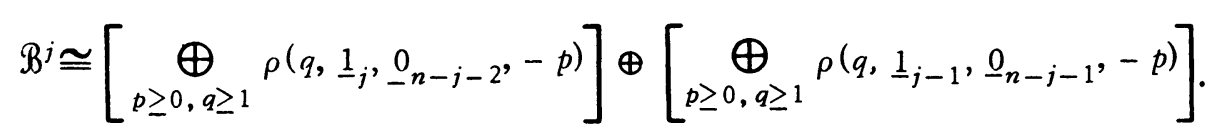

Proof. This is just a matter of combining Theorem 1, the Frobenius Reciprocity The orem, the Branching Theorem, and the observation that the $j$ th exterior power of the standard representation of $U(n-1)$ on $\mathrm{C}^{n-1}$ is $\rho\left(\underline{1}_{j}, \underline{0}_{n-j-1}\right)$. Thus $\rho\left(m_{1}, \cdots, m_{n}\right)$ occurs in $\mathfrak{B}^{0}$ (and with multiplicity one) if and only if $m_{1} \geq$ $0 \geq \cdots \geq 0 \geq m_{n}$; it occurs in $\mathfrak{B}^{n-1}$ if and only if $m_{1} \geq 1 \geq \cdots \geq 1 \geq m_{n}$; and it occurs in $\mathscr{B}^{j}(1 \leq j \leq n-2)$ if and only if $m_{1} \geq 1 \geq \cdots \geq 1 \geq m_{j+1} \geq 0 \geq \cdots \geq 0$ $\geq m_{n}$. Setting $m_{1}=q, m_{n}=-p$, we obtain the theorem. Q.E.D. 
We shall denote by $\Phi_{p q j}$ (respectively $\Psi_{p q j}$ ) the subspace of $\mathfrak{B}^{j}$ corresponding to the representation $\rho\left(q, \underline{1}_{j}, \underline{0}_{n-j-2},-p\right)$ (respectively $\rho\left(q, \underline{1}_{j-1}, \underline{0}_{n-j-1},-p\right)$ ). Thus $\mathfrak{B}^{0}=\bigoplus_{p \geq 0, q \geq 0} \Phi_{p q 0}, \mathfrak{B}^{n-1}=\bigoplus_{p \geq-1, q \geq 1} \Psi_{p q(n-1)}$, and $\mathscr{B}^{j}=$ $\bigoplus_{p \geq 0, q \geq 1}\left[\Phi_{p q j} \oplus \Psi_{p q j}\right]$ for $1 \leq j \leq n-2$. Our next task will be to identify these subspaces explicity.

Recall that the space $V\left(q, \underline{1}_{k}, \underline{0}_{n-k-2},-p\right)(q \geq 1, p \geq 0,0 \leq k \leq n-2)$ on which $\rho\left(q, \underline{1}_{k}, \underline{0}_{n-k-2},-p\right)$ acts is a subspace of

$$
C_{p q k}=\left[\otimes^{p} C^{n *}\right] \otimes\left[\wedge^{k+1} C^{n}\right] \otimes\left[\otimes^{q-1} C^{n}\right]
$$

likewise $V\left(\underline{0}_{n-1}, p\right)$ is a subspace of $C_{p 00}=\boldsymbol{Q}^{p} \mathbf{C}^{n^{*}}$ and $V\left(q, \underline{1}_{n-2}\right)$ is a subspace of

$$
C_{(-1) q(n-2)}=\left[\wedge^{n} \mathrm{C}^{n}\right] \otimes\left[\otimes^{q-1} \mathrm{C}^{n}\right]
$$

We define linear maps $F_{p q j}: C_{p q j} \rightarrow \mathfrak{B}^{j}$ and $G_{p q j}: C_{p q(j-1)} \rightarrow \mathfrak{B}^{j}$ as follows. If $\left\{e_{i}: 1 \leq i \leq n\right\}$ is the canonical bas is for $\mathrm{C}^{n}$, then

$$
\begin{aligned}
\left\{e _ { a _ { 1 } } ^ { * } \otimes \cdots \otimes e _ { a _ { p } } ^ { * } \otimes \left(e_{b_{1}}\right.\right. & \left.\wedge \cdots \wedge e_{b_{k+1}}\right) \otimes e_{c_{1}} \otimes \cdots \otimes e_{c_{q-1}}: \\
1 & \left.\leq a_{i} \leq n, 1 \leq b_{1}<\cdots<b_{k+1} \leq n, 1 \leq c_{i} \leq n\right\}
\end{aligned}
$$

is a basis for $C_{p q k}(p \geq 0, q \geq 1)$, and

$$
\begin{gathered}
\left\{e_{a_{1}}^{*} \otimes \cdots \otimes e_{a_{p}}^{*}: 1 \leq a_{i} \leq n\right\}, \\
\left\{\left(e_{1} \wedge \cdots \wedge e_{n}\right) \otimes e_{c_{1}} \otimes \cdots \otimes e_{c_{q-1}}: 1 \leq c_{i} \leq n\right\}
\end{gathered}
$$

are bases for $C_{p 00}$ and $C_{(-1) q(n-2)}$ respectively. $F_{p q j}$ and $G_{p q j}$ are defined on these bases by

$$
\begin{gathered}
F_{p q j}\left(e_{a_{1}}^{*} \otimes \cdots \otimes e_{a_{p}}^{*} \otimes\left(e_{b_{1}} \wedge \cdots \wedge e_{b_{j+1}}\right) \otimes e_{c_{1}} \otimes \cdots \otimes e_{c_{q-1}}\right) \\
=z_{a_{1}} \cdots z_{a_{p} \bar{z}_{c_{1}}} \cdots \bar{z}_{c_{q-1}} \sum_{i+1}^{j+1}(-1)^{i-1} \bar{z}_{b_{i} \zeta_{b_{1}}} \wedge \cdots \hat{\zeta}_{b_{i}} \cdots \wedge \zeta_{b_{j+1}}, \\
G_{p q j}\left(e_{a_{1}}^{*} \otimes \cdots \otimes e_{a_{p}}^{*} \otimes\left(e_{b_{1}} \wedge \cdots \wedge e_{b_{j}}\right) \otimes e_{c_{1}} \otimes \cdots \otimes e_{c_{q-1}}\right) \\
=z_{a_{1}} \cdots z_{a_{p}} \bar{z}_{c_{1}} \cdots \bar{z}_{c_{q-1}} \zeta_{1} \wedge \cdots \wedge \zeta_{j}
\end{gathered}
$$

for $q \geq 1, p \geq 0$, and 


$$
\begin{gathered}
F_{p 00}\left(e_{a_{1}}^{*} \otimes \cdots \otimes e_{a_{p}}^{*}\right)=z_{a_{1}} \cdots z_{a_{p}} \\
G_{(-1) q(n-1)}\left(\left(e_{1} \wedge \cdots \wedge e_{n}\right) \otimes e_{c_{1}} \otimes \cdots \otimes e_{c_{q-1}}\right) \\
=\bar{z}_{c_{1}} \cdots \bar{z}_{c_{q-1}} \sum_{i=1}^{n}(-1)^{i+n} \bar{z}_{i} \zeta_{1} \wedge \cdots \hat{\zeta}_{i} \cdots \wedge \zeta_{n} .
\end{gathered}
$$

Theorem 3. $F_{p q j} \mid V\left(q, \underline{1}_{j}, \underline{0}_{n-j-2},-p\right)$ and $G_{p q j} \mid V\left(q, \underline{1}_{j-1}, \underline{0}_{n-j-1},-p\right)$ are nonzero and commute with the action of $U(n)$. Therefore (by Schur's lemma) their ranges are $\Phi_{p q j}$ and $\Psi_{p q j}$, respectively, and they are isomorphisms of irreducible $U(n)$-modules.

Proof: We see that $F_{p q j} \mid V\left(q, \underline{1}_{j}, \underline{0}_{n-j-2},-p\right)$ and $G_{p q j} \mid V\left(q, \underline{1}_{j-1}, \underline{0}_{n-j-1},-p\right)$ are nonzero by observing that the primitive vectors $P\left(q, \underline{1}_{j}, \underline{0}_{n-j-2},-p\right)$ and $P\left(q, \underline{1}_{j-1}, \underline{0}_{n-j-1},-p\right)$ are among the basis elements for $C_{p q j}$ and $C_{p q(j-1)}$, and their images are clearly nonzero. Showing that $F_{p q j}$ and $G_{p q j}$ commute with the action of $U(n)$ is just a matter of unraveling the definitions. We observe that the action of $U(n)$ on the coordinate functions $z_{i}$ is the contragradient of the standard action on $\mathrm{C}^{n}$, and the action on the conjugate functions $\bar{z}_{i}$ and their differentials $d \bar{z}_{i}$ is therefore the standard action on $\mathbf{C}^{n}$. Moreover, the action on the $\zeta_{i}$ 's is the standard action on $\mathrm{C}^{n}$ since $\zeta_{i}=d \bar{z}_{i}-2 \bar{z}_{i} \bar{\partial} r$ and $\bar{\partial} r$ is invariant. Finally, the mappings taking $e_{b_{1}} \wedge \cdots \wedge e_{b_{j}}$ to $\zeta_{b_{1}} \wedge \cdots \wedge \zeta_{b_{j}}$ or

$$
\sum_{i=1}^{j}(-1)^{i-1} \bar{z}_{b_{i}} \zeta_{b_{1}} \wedge \cdots \hat{\zeta}_{b_{i}} \cdots \wedge \zeta_{b_{j}}
$$

preserve the skew-symmetry, as is easily verified. The theorem follows by putting these facts together with the definition of tensor product representation; details are left to the reader. Q.E.D.

We define the forms $\phi_{p q j}$ and $\psi_{p q j}$ to be the images under $F_{p q j}$ and $G_{p q j}$ of the primitive vectors $P\left(q, \underline{1}_{j}, \underline{0}_{n-j-2},-p\right)$ and $P\left(q, \underline{1}_{j-1}, \underline{0}_{n-j-1},-p\right)$; thus

$$
\begin{aligned}
\phi_{p q j} & =\bar{z}_{1}^{q-1} z_{n}^{p} \sum_{i=1}^{j+1}(-1)^{i-1} \bar{z}_{i} \zeta_{1} \wedge \cdots \hat{\zeta}_{i} \cdots \wedge \zeta_{j+1} \quad(q \geq 1, p \geq 0), \\
\psi_{p q j} & =\bar{z}_{1}^{q-1} z_{n}^{p} \zeta_{1} \wedge \cdots \wedge \zeta_{j} \quad(q \geq 1, p \geq 0), \\
\phi_{p 00} & =z_{n}^{p} \quad(p \geq 0), \\
\psi_{(-1) q(n-1)} & =\bar{z}_{1}^{q-1} \sum_{i=1}^{n}(-1)^{i+n} \bar{z}_{i} \zeta_{1} \wedge \cdots \hat{\zeta}_{i} \cdots \wedge \zeta_{n} \quad(q \geq 1) .
\end{aligned}
$$


These forms will play a crucial role in our analysis. The rather peculiar definition of $\psi_{(-1) q(n-1)}$ is explained by the easily verified fact that

$$
\zeta_{1} \wedge \cdots \wedge \zeta_{n-1}=z_{n} \sum_{i=1}^{n}(-1)^{i+n} \bar{z}_{i} \zeta_{1} \wedge \cdots \hat{\zeta}_{i} \cdots \wedge \zeta_{n}
$$

Thus we could also write

$$
\psi_{(-1) q(n-1)}=\bar{z}_{1}^{q-1} z_{n}^{-1} \zeta_{1} \wedge \cdots \wedge \zeta_{n-1}
$$

which is consistent with the definition of the other $\psi_{p q j}$ 's.

4. Further remarks. Let us take a closer look at the space $B^{0}$ of functions on $S_{n}$. The primitive vectors $\phi_{p q 0}=\bar{z}_{1}^{q} z_{n}^{p}$ are the restrictions to $S_{n}$ of harmonic polynomials on $\mathrm{C}^{n}$, as the Euclidean Laplace operator is

$$
4 \sum_{i=1}^{n} \frac{\partial^{2}}{\partial z_{i} \partial \bar{z}_{i}}
$$

Since this operator commutes with the action of $U(n)$, and this action preserves homogeneity, it is clear that $\Phi_{p q 0}$ consists entirely of harmonic polynomials of degree $p$ in the $z_{i}$ 's and the degree $q$ in the $\bar{z}_{i}$ 's. Moreover, since the space $\mathcal{H}^{p q}$ of all such polynomials transforms under $U(n)$ via the representation $\rho\left(q, \underline{0}_{n-2},-p\right)$ and this representation occurs with multiplicity one in $\mathfrak{B}^{0}$, we see that $\Phi_{p q 0}=\mathcal{H}^{p q}$. Therefore we have obtained a refinement of the usual decomposition of functions on the sphere into spherical harmonics (cf. Müller [13]): we have bigraded the spherical harmonics according to their holomorphic and antiholomorphic degrees.

We may make some more interesting observations by considering the special unitary group $S U(n)$. Since $S_{n} \cong S U(n) / S U(n-1)$, we could have carried through all the preceding discussion in the context of $S U(n)$ and obtained the decomposition of $\Re^{j}$ by the representations of this group. Since the irreducible representations of $S U(n)$ are the same as those of $U(n)$ modulo powers of the determinant, the results are essentially the same. However, two points deserve mention.

First, the spaces $\mathfrak{B}^{0}$ and $\mathfrak{B}^{n-1}$ are isomorphic as $S U(n)$-modules. Indeed, $\rho\left(q, \underline{0}_{n-2}, p\right)=(\mathrm{det})^{-1} \rho\left(q+1, \underline{1}_{n-2},-(p-1)\right)$, so $\Phi_{p q 0} \cong \Psi_{(p-1)(q+1)(n-1)}$, and this isomorphism is displayed on the primitive vectors by the correspondence

$$
\begin{aligned}
& \bar{z}_{1}^{q} z_{n}^{p} \leftrightarrow \bar{z}_{1}^{q} z_{n}^{p-1} \zeta_{1} \cdots \wedge \zeta_{n-1} \\
&=\bar{z}_{1}^{q} z_{n}^{p} \sum_{i=1}^{n}(-1)^{i+n} \bar{z}_{i} \zeta_{1} \wedge \cdots \hat{\zeta}_{i} \cdots \wedge \zeta_{n}
\end{aligned}
$$

The form 


$$
\sum_{i=1}^{n}(-1)^{i+n} \bar{z}_{i} \zeta_{1} \wedge \cdots \hat{\zeta}_{i} \cdots \wedge \zeta_{n}
$$

is invariant under $S U(n)$ and plays the role of a constant function.

Second, in the case $n=2$, we have $S_{n} \cong S U(2) / S U(1)=S U(2)$; the representations $\rho(q,-p) \mid S U(2)$ exhaust the irreducible representations of $S U(2)$ with $\rho(q,-p)\left|S U(2)=\rho\left(q^{\prime},-p^{\prime}\right)\right| S U(2)$ if and only if $p+q=p^{\prime}+q^{\prime}$; and $\operatorname{dim} \rho(q,-p)$ $=p+q+1$ (as is easily verified). Therefore we have recovered the Peter-Weyl the orem for $S U(2)$ : each irreducible representation occurs in the left regular representation with multiplicity equal to its dimension.

5. The $\bar{\partial}_{b}$ operator. The decomposition of the spaces $\mathfrak{B}^{j}$ allows us to calculate the action of $\bar{\partial}_{b}$ explicitly. By Schur's lemma, since $\bar{\partial}_{b}$ commutes with the action of $U(n)$, on each irreducible subspace it must either be zero or an isomorphism onto an irreducible subspace of the same type. In fact, we shall have no trouble in seeing that $\bar{\partial}_{b}$ is an isomorphism whenever it can be and is zero precisely when there is no isomorphic subspace for it to map into.

$\Psi$

Theorem 4. $\bar{\partial}_{b}\left(\Phi_{p 00}\right)=0, \bar{\partial}_{b}\left(\Psi_{p q j}\right)=0$, and for $p \geq 0, q \geq 1, \bar{\partial}_{b}\left(\Phi_{p q j}\right)=$ $p q(j+1)^{\circ}$

Proof. By Schur's lemma, to prove the last statement it suffices to show that $\bar{\partial}_{b}$ is nonzero on $\Phi_{p q j}$. We check it on the primitive vector:

$$
\begin{aligned}
\bar{\partial}_{b} \phi_{p q j} & =\bar{\partial}_{b}\left(\bar{z}_{1}^{q-1} z_{n}^{p} \sum_{i=1}^{j+1}(-1)^{i-1} \bar{z}_{i} \zeta_{1} \wedge \cdots \hat{\zeta}_{i} \cdots \wedge \zeta_{j+1}\right) \\
& =q \bar{z}_{1}^{q-1} z_{n}^{p} \zeta_{1} \wedge \cdots \wedge \zeta_{j+1}+\bar{z}_{1}^{q-1} z_{n}^{p} \sum_{i=2}^{j+1}(-1)^{i-1} \zeta_{i} \wedge \zeta_{1} \wedge \cdots \hat{\zeta}_{i} \cdots \wedge \zeta_{j+1} \\
& =(q+j) \bar{z}_{1}^{q-1} z_{n}^{p} \zeta_{1} \wedge \cdots \wedge \zeta_{j+1} \\
& =(q+j) \psi_{p q(j+1)} \neq 0 \quad \text { since } q+j>0 .
\end{aligned}
$$

Schur's lemma also forces the first two statements to be true, but we can now also see this directly. $\bar{\partial}_{b}\left(\Phi_{p 00}\right)=0$ because $\Phi_{p 00}$ consists of holomorphic polynomials; $\bar{\partial}_{b}\left(\Psi(-1) q\left(n-\frac{1}{\partial}\right)=0\right.$ because $\bar{\partial}_{b}=0$ on $\mathbb{B}^{n-1}$; and for $p \geq 0, q \geq 1$, $\bar{\partial}_{b}\left(\Psi_{p q j}\right)=0$ because $\bar{\partial}_{b}^{2}=0$ and $\Psi_{p q j}=\bar{\partial}_{b}\left(\Phi_{p q(j-1)}\right)$. Q.E.D.

Corollary. The complex

$$
0 \rightarrow \mathfrak{B}^{0} \stackrel{\bar{\partial}_{b}}{\longrightarrow} \mathfrak{B}^{1} \stackrel{\bar{\partial}_{b}}{\longrightarrow} \cdots \stackrel{\bar{\partial}_{b}}{\longrightarrow} \mathfrak{B}^{n-1} \longrightarrow 0
$$

is exact at $\mathfrak{B}^{j}$ for $1 \leq j \leq n-2$, and the cobomology is $\bigoplus_{p \geq 0} \Phi_{p 00}$ at $\mathfrak{B}^{0}$ and $\bigoplus_{q \geq 1} \Psi_{(-1) q(n-1)}$ at $\mathfrak{B}^{n-1}$ 
6. Computation of the eigenvalues. We have seen that $\bar{\partial}_{b}\left(\Psi_{p q j}\right)=0$, $\bar{\partial}_{b}\left(\Phi_{p 00}\right)=0$, and $\bar{\partial}_{b}: \Phi_{p q j} \rightarrow \Psi_{p q(j+1)}$ is a constant multiple of a unitary map for $p \geq 0, q \geq 1$. This constant is determined up to a complex factor of modulus one and so can be taken to be real and positive; when so determined, it will be called the eigenvalue of $\bar{\partial}_{b}$ on $\Phi_{p q j}$. The proof of Theorem 4 shows that this eigenvalue is $(q+j)\left\|\psi_{p q(j+1)}\right\| /\left\|\phi_{p q j}\right\|$. We are therefore faced with the task of computing the norms of the primitive vectors.

We cannot simply take the norms of the coefficients, for the $\zeta_{i_{1}} \wedge \ldots \wedge \zeta_{i_{j}}$ 's are not a basis, much less an orthonormal one. However, the bundle $B^{0 j}$ is a subbundle of $A^{0 j}\left|S_{n}=\bigwedge^{j} \bar{T}^{*} \mathrm{C}^{n}\right| S_{n}$, and this bundle has a nice pointwise-orthonormal basis for its sections (as a module over the functions), namely $\left\{2^{-k / 2} d \bar{z}_{i_{1}} \wedge\right.$ $\left.\ldots \wedge d \bar{z}_{i_{j}}: 1 \leq i_{1}<\cdots<i_{j} \leq n\right\}$. We therefore express the $\phi$ 's and $\psi$ 's in terms of this basis.

For $2 \leq j \leq n, 1 \leq i_{1}, \cdots, i_{j} \leq n$, set

$$
\omega_{i_{1}} \cdots i_{j}=\sum_{a=1}^{j}(-1)^{j-a} \bar{z}_{i_{a}} d \bar{z}_{i_{1}} \wedge \cdots \widehat{d}_{i_{a}} \cdots \wedge d \bar{z}_{i_{j}}
$$

Lemma 1. $\omega_{i_{1}} \cdots_{i_{j}}$ is alternating in the indices $i_{1}, \cdots, i_{j}$.

The verification is left to the reader.

Lemma 2. $\zeta_{i_{1}} \wedge \cdots \wedge \zeta_{i_{j}}=\sum_{a=1}^{n} z_{a} \omega_{i_{1}} \cdots_{i_{j}} a$

Proof. Let $\rho=2 \bar{\partial} r=\Sigma_{1}^{n} z_{a} d \bar{z}_{a}$. Then, using the fact that $\Sigma_{1}^{n} z_{a} \bar{z}_{a}=1$,

$$
\begin{aligned}
& \zeta_{i_{1}} \wedge \cdots \wedge \zeta_{i_{j}}=\left(d \bar{z}_{i_{1}}-\bar{z}_{i_{1}} \rho\right) \wedge\left(d \bar{z}_{i_{2}}-\bar{z}_{i_{2}} \rho\right) \wedge \cdots \wedge\left(d \bar{z}_{i_{j}}-\bar{z}_{i_{j}} \rho\right) \\
& =d \bar{z}_{i_{1}} \wedge \cdots \wedge d \bar{z}_{i_{i}}-\left(\bar{z}_{i_{1}} \rho\right) \wedge d \bar{z}_{i_{2}} \wedge \cdots \wedge d \bar{z}_{i_{j}}-\cdots-d \bar{z}_{i_{1}} \wedge \cdots \wedge d \bar{z}_{i_{j-1}} \wedge\left(\bar{z}_{i_{j}} \rho\right) \\
& =d \bar{z}_{i_{1}} \wedge \cdots \wedge d \bar{z}_{i_{j}}+\left[\sum_{b=1}^{j}(-1)^{j-b+1} \bar{z}_{i_{b}} d \bar{z}_{i_{1}} \wedge \cdots \widehat{d \bar{z}}_{i_{b}} \cdots \wedge d \bar{z}_{i_{j}}\right] \wedge \rho \\
& =\sum_{a=1}^{n} z_{a} \bar{z}_{a} d \bar{z}_{i_{1}} \wedge \cdots \wedge d \bar{z}_{i_{j}} \\
& +\sum_{a=1}^{n} z_{a} \sum_{b=1}^{j}(-1)^{j-b+1} \bar{z}_{i_{b}} d \bar{z}_{i_{1}} \wedge \cdots \widehat{d \bar{z}}_{i_{b}} \cdots \wedge d \bar{z}_{i_{j}} \wedge d \bar{z}_{a} \\
& =\sum_{a=1}^{n} z_{a}\left[\sum_{b=1}^{j}(-1)^{j-b+1} \bar{z}_{i_{b}} d \bar{z}_{i_{1}} \wedge \cdots \widehat{d}_{i_{b}} \cdots \wedge d \bar{z}_{i_{j}} \wedge d \bar{z}_{a}+\bar{z}_{a} d \bar{z}_{i_{1}} \wedge \cdots \wedge d \bar{z}_{i_{j}}\right] \\
& =\sum_{a=1}^{n} z_{a^{\prime}} \omega_{i_{1}} \cdots i_{j} a \cdot \quad \text { Q.E.D. }
\end{aligned}
$$


As a corollary, we have

$$
\begin{aligned}
\zeta_{1} \wedge \cdots \wedge \zeta_{j}= & \sum_{a=1}^{n} z_{a} \omega_{1} \cdots j a \\
& =\sum_{a=j+1}^{n} z_{a} \omega_{1} \cdots j a \text { (by Lemma 1) } \\
& =\sum_{a=j+1}^{n} z_{a}\left[\sum_{b=1}^{j}(-1)^{j-b+1} \bar{z}_{b} d \bar{z}_{1} \wedge \cdots \widehat{z}_{b} \cdots \wedge d \bar{z}_{j} \wedge d \bar{z}_{a}\right. \\
& \left.=\sum_{a=j+1}^{n} z_{a} \bar{z}_{a} d \bar{z}_{1} \wedge \cdots \wedge \bar{z}_{a} d \bar{z}_{1} \wedge \cdots \wedge d \bar{z}_{j}\right] \\
& +\sum_{a=j+1}^{n} \sum_{b=1}^{j}(-1)^{j-b+1} \bar{z}_{b} z d \bar{z}_{1} \wedge \cdots \widehat{d \bar{z}}_{b} \cdots \wedge d \bar{z}_{j} \wedge d \bar{z}_{a} .
\end{aligned}
$$

Lemma 3.

$$
\sum_{i=1}^{j}(-1)^{i-1} \bar{z}_{i} \zeta_{1} \wedge \cdots \hat{\zeta}_{i} \cdots \wedge \zeta_{j}=\sum_{i=1}^{j}(-1)^{i-1} \bar{z}_{i} d \bar{z}_{1} \wedge \cdots \widehat{d}_{i} \cdots \wedge d \bar{z}_{j}
$$

Proof. Using Lemmas 1 and 2, we find that

$$
\begin{aligned}
& \sum_{i=1}^{j}(-1)^{i-1} \bar{z}_{i} \zeta_{1} \wedge \cdots \hat{\zeta}_{i} \cdots \wedge \zeta_{j} \\
& \quad=\sum_{j=1}^{j} \sum_{a=j+1}^{n}(-1)^{i-1} \bar{z}_{i} z_{a} \omega_{1} \cdots \hat{i} \cdots j a \\
&
\end{aligned}
$$

The formula is then proved by expanding the $\omega$ 's in terms of the $d \bar{z}_{i}$, s, collecting terms, and using the fact that $\Sigma_{1}^{n} z_{a} \bar{z}_{a}=1$. Q. E. D.

There now follows immediately:

Theorem 5.

$$
\begin{aligned}
\phi_{p q j}=\bar{z}_{1}^{q-1} z_{n}^{p} & \sum_{i=1}^{j+1}(-1)^{i-1} d \bar{z}_{1} \wedge \ldots \widehat{d}_{i} \cdots \wedge d \bar{z}_{j+1}, \\
\psi_{p q j}=\bar{z}_{1}^{q-1} z_{n}^{p} & {\left[\left(\sum_{a=j+1}^{n} z_{a} \bar{z}_{a}\right) d \bar{z}_{1} \wedge \cdots \wedge d \bar{z}_{j}\right.} \\
& \left.+\sum_{a=j+1}^{n} \sum_{b=1}^{j}(-1)^{j-b+1} \bar{z}_{b} z_{a} d \bar{z}_{1} \wedge \cdots \widehat{d}_{b} \cdots \wedge d \bar{z}_{j} \wedge d \bar{z}_{a}\right] .
\end{aligned}
$$


Thus

$$
\begin{aligned}
2^{-j}\left\|\phi_{p q j}\right\|^{2}= & \sum_{i=1}^{j+1} \int\left|\bar{z}_{1}^{q-1} z_{n}^{p} \bar{z}_{i}\right|^{2}=\sum_{i=1}^{j+1} \int\left|z_{1}^{q-1} z_{n}^{p} z_{i}\right|^{2}, \\
2^{-(j+1)}\left\|\psi_{p q(j+1)}\right\|^{2}= & \int\left|\bar{z}_{1}^{q-1} z_{n}^{p} \sum_{a=j+2}^{n} z_{a} \bar{z}_{a}\right|^{2}+\sum_{a=j+2}^{n} \sum_{b=1}^{j+1} \int\left|\bar{z}_{1}^{q-1} z_{n}^{p} \bar{z}_{b} z_{a}\right|^{2} \\
= & \sum_{a=j+2}^{n} \int\left|z_{1}^{q-1} z_{n}^{p} z_{a}^{2}\right|^{2}+2 \sum_{j+2 \leq b<a \leq n} \int\left|z_{1}^{q-1} z_{n}^{p} z_{a} z_{b}\right|^{2} \\
& +\sum_{a=j+2}^{n} \sum_{b=1}^{j+1} \int\left|z_{1}^{q-1} z_{n}^{p} z_{b} z_{a}\right|^{2} .
\end{aligned}
$$

We are therefore reduced to computing integrals of the type $\int\left|z^{\alpha}\right|^{2}$ where $\alpha$ is a multi-index. This may most easily be accomplished by the following bit of trickery, which was pointed out to me by V. Bargmann and E. Nelson. Consider $I=\int_{C^{n}}\left|z^{\alpha}\right|^{2} \exp \left(-|z|^{2}\right)$. In rectangular coordinates,

$$
I=\prod_{1}^{n} \int_{C}\left|z_{i}^{\alpha_{i}}\right|^{2} \exp \left(-\left|z_{i}\right|^{2}\right)=\prod_{1}^{n} \int_{0}^{2 \pi} \int_{0}^{\infty} \exp \left(-r^{2}\right) r^{2 a_{i}+1} d r d \theta
$$

In spherical coordinates,

$$
I=\int_{0}^{\infty} \exp \left(-r^{2}\right) r^{2|\alpha|+2 n-1} d r \int_{S_{n}}\left|z^{a}\right|^{2}
$$

Since $\int_{0}^{\infty} \exp \left(-r^{2}\right) r^{2 m+1} d r=1 / 2 m$ !, we therefore have

$$
\int_{S_{n}}\left|z^{\alpha}\right|^{2}=\frac{\Pi_{1}^{n}\left[2 \pi^{1 / 2} \alpha_{i} !\right]}{1 / 2(|\alpha|+n-1) !}=\frac{2 \pi^{n} \alpha !}{(|\alpha|+n-1) !} .
$$

Thus

$$
\begin{aligned}
\left\|\phi_{p q j}\right\|^{2} & =2^{j}\left[\int\left|z_{1}^{q} z_{n}^{p}\right|^{2}+\sum_{i=2}^{j+1} \int\left|z_{1}^{q-1} z_{n}^{p} z_{i}\right|^{2}\right] \\
& =2^{j}\left[\frac{2 \pi^{n} p ! q !}{(p+q+n-1) !}+j \frac{2 \pi^{n} p !(q-1) !}{(p+q+n-1) !}\right]=\frac{2^{j+1} \pi^{n} p !(q-1) !}{(p+q+n-1) !}(q+j) .
\end{aligned}
$$

Likewise, keeping in mind that the terms with $a=n$ or $b=1$ in the expression for $\left\|\psi_{p q(j+1)}\right\|$ are of a different form than the others, we find after some simple calculations that 


$$
\left\|\psi_{p q(j+1)}\right\|^{2}=\frac{2^{j+2} \pi^{n} p !(q-1) !}{(p+q+n-1) !}(p+n-j-1) .
$$

We have now proved the main theorem.

Theorem 6. The eigenvalue of $\bar{\partial}_{b}$ on $\Phi_{p q j}$ is

$$
\gamma_{p q j}=(q+j)\left\|\psi_{p q(j+1)}\right\| /\left\|\phi_{p q j}\right\|=[2(q+j)(p+n-j-1)]^{1 / 2} .
$$

Notice that $\gamma_{p q j}=\gamma_{p q(n-j-1)}$. Thus in some sense the $\bar{\partial}_{b}$ complex is symmetric with respect to holomorphicity and antiholomorphicity.

$\bar{\partial}_{b}$ is a weighted shift operator on $\bigoplus \mathfrak{B}^{j}$; therefore its adjoint $\widehat{b}_{b}$ is also a weighted shift operator with the same weights but shifting in the other direction. Thus $\delta_{b}\left(\Phi_{p q j}\right)=0, \delta_{b}\left(\Psi_{(-1) q(n-1)}\right)=0$, and $\delta_{b}$ maps $\Psi_{p q j}(p \geq 0)$ onto $\Phi_{p q(j-1)}$ with eigenvalue $\gamma_{p q(j-1)}$. From this it follows that the $\Phi_{p q j}$ 's and $\Psi_{p q j}$ 's are eigenspaces of $\square_{b}$, and the eigenvalues of $\square_{b}$ are zero on $\Phi_{p 00}$ and $\Psi_{(-1) q(n-1)}$ and $\gamma_{p q j}^{2}$ on $\Phi_{p q j}$ and $\Psi_{p q(j+1)}(p \geq 0, q \geq 1)$. The $\Phi$ 's and $\Psi$ 's are also eigenspaces of $G_{b}$, the Green's operator defined by $G_{b}=0$ on the null space of $\square_{b}$ and $G_{b}=\square_{b}^{-1}$ on the orthogonal complement, and the eigenvalues of $G_{b}$ are zero on $\Phi_{p 00}$ and $\Psi_{(-1) q(n-1)}$ and $\gamma_{p q j}^{-2}$ on $\Phi_{p q j}$ and $\Psi_{p q(j+1)}$ $(p \geq 0, q \geq 1)$.

Theorem 7. $\bar{\partial}_{b}, \delta_{b}$, and $\square_{b}$ bave closed ranges, and $G_{b}$ is compact.

Proof. The first assertion follows from the fact that the nonzero eigenvalues are bounded away from zero. Also, only finitely many of the $\gamma_{p q j}^{-2}$ are greater than any fixed constant, and each of them is the eigenvalue for a finite-dimensional eigenspace. Therefore $G_{b}$ is the norm limit of operators of finite rank and hence is compact. Q.E.D.

It should be noted that the closed range property is strictly a global one. If one restricts to a small open set in $S_{n}$, the situation may be quite different, as is shown by the Lewy example (cf. Chapter I).

Let us now form a complete orthonormal basis for $\bigoplus \mathfrak{B}^{j}$. Set $\phi_{p q j}^{1}=$ $\phi_{p q j} /\left\|\phi_{p q j}\right\|$, and extend this to an orthonormal basis $\left\{\phi_{p q j}^{a}: 1 \leq a \leq \operatorname{dim} \Phi_{p q j}\right\}$ for $\Phi_{p q j}$; likewise let $\left\{\psi_{(-1) q(n-1)}^{a}: 1 \leq a \leq \operatorname{dim} \Psi_{(-1) q(n-1)}\right\}$ be an orthonormal basis for $\Psi_{(-1) q(n-1)}$ with $\psi_{(-1) q(n-1)}^{1}=\psi_{(-1) q(n-1)} /\left\|\psi_{(-1) q(n-1)}\right\|$. Set $\psi_{p q j}^{a}=\gamma_{p q(j-1)}^{-1} \bar{\partial}_{b} \phi_{p q(j-1)}^{a}$ for $p \geq 0, q \geq 1$; in particular, $\psi_{p q j}^{1}=\psi_{p q j} /\left\|\psi_{p q j}\right\|$. Then $\left\{\psi_{p q j}^{a}\right\}$ is an orthonormal basis for $\Psi_{p q j}$. We have $\bar{\partial}_{b} \phi_{p q j}^{a}=\gamma_{p q j}{ }_{p q(j+1)}^{a}$, $\bar{\partial}_{b} \psi_{p q j}^{a}=0, \delta_{b} \phi_{p q j}^{a}=0, \delta_{b} \psi_{p q j}^{a}=\gamma_{p q(j-1)} \phi_{p q(j-1)}^{a}, \square_{b} \phi_{p q j}^{a}=\gamma_{p q j}^{2} \phi_{p q j}^{a}$, and $\square_{b} \psi_{p q j}^{a}=\gamma_{p q(j-1)}^{2} \psi_{p q j}^{a}$. Thus $\left\{\phi_{p q j}^{a}\right\}_{p q j a} \cup\left\{\psi_{p q j}^{a}\right\}_{p q j a}$ forms a canonical basis for the $\bar{\partial}_{b}$ complex in the sense of Kodaira (cf. Kodaira and Spencer [5]). 


\section{FIBER BUNDLES \\ (CONNECTION WITH DOLBEAULT COMPLEXES ON $C P^{n-1}$ )}

If we consider the circle group $S_{1}$ embedded in $U(n)$ as its center, i.e. as multiples of the identity, the quotient of $S_{n}$ by the $S_{1}$ action is the complex manifold $C P^{n-1}$. Since the line subbundle of $\bar{T}^{*} \mathrm{C}^{n} \mid S_{n}$ spanned by $\overline{\partial r}$ is the part of $\bar{T}^{*} \mathrm{C}^{n} \mid S_{n}$ which is left out of the $\bar{\lambda}_{b}$ complex, and this is also the cokernel of the pullback of $\bar{T}^{*} \mathbf{C} P^{n-1}$ via the projection, it is strongly suggested that there should be an intimate connection between the $\bar{\partial}_{b}$ complex on $S_{n}$ and the $\bar{\partial}$ complex on $\mathrm{C} P^{n-1}$. This is indeed the case.

That there should be such a relationship was first pointed out to me by H. Pittie, and it was M. F. Atiyah who showed me how to express it in terms of line bundles on $\mathrm{C} P^{n-1}$.

In order to study the behavior of the $\bar{\partial}_{b}$ complex under the action of $S_{1}$, we need to know how the representations $\rho\left(m_{1}, \cdots, m_{n}\right)$ decompose when restricted to $S_{1}$. Recall that the irreducible representations of $S_{1}=U(1)$ are $\{\rho(m): m \in \mathbf{Z}\}$ where $\rho(m)\left(e^{i \theta}\right)$ acts on $\mathbf{C}$ by multiplication by $e^{i m \theta}$.

Lemma 1. $\rho\left(m_{1}, \cdots, m_{n}\right) \mid S_{1}=\left(\operatorname{dim} \rho\left(m_{1}, \cdots, m_{n}\right)\right) \rho\left(\Sigma_{1}^{n} m_{i}\right)$.

Proof. Since $S_{1}$ is the center of $U(n)$, by Schur's lemma it acts as multiples of the identity on the representation space of $\rho\left(m_{1}, \cdots, m_{n}\right)$, so $\rho\left(m_{1}, \cdots, m_{n}\right) \mid S_{1}$ is the sum of $\operatorname{dim} \rho\left(m_{1}, \cdots, m_{n}\right)$ copies of some $\rho(M)$. Now $\rho\left(m_{1}, \cdots, m_{n}\right)=$ $(\text { det })^{m_{n}} \rho\left(m_{1}-m_{n}, \cdots, m_{m-1}-m_{n}, 0\right)$, and $\rho\left(m_{1}-m_{n}, \cdots, m_{n-1}-m_{n}, 0\right)$ is a subrepresentation of the standard representation of $U(n)$ on $\otimes^{K} \mathbf{C}^{n}, K=$ $\Sigma_{1}^{n-1}\left(m_{i}-m_{n}\right)=\Sigma_{1}^{n} m_{i}-n m_{n}$. By construction of this representation, $S_{1}$ acts on $\otimes^{K} C^{n}$ via $\rho(K)$. On the other hand, $\operatorname{det} \mid S_{1}=\rho(n)$, so finally we see that $M=$ $K+n m_{n}=\Sigma_{1}^{n} m_{i}$. Q.E.D.

From this lemma and the results of Chapter II, $\$ 3$ and $\$ 5$, we can immediately read off the action of $S_{1}$ on the $\bar{\partial}_{b}$ complex.

Theorem 1. For each $m \in \mathbf{Z}$, set $\mathfrak{B}^{0}(m)=\bigoplus_{q-p=m} \Phi_{p q 0}, \mathfrak{B}^{n-1}(m)=$ $\bigoplus_{q-p+n-2=m} \Psi_{p q(n-1)}$, and for $1 \leq j \leq n-2, \mathcal{B}^{j}(m)=\left[\bigoplus_{q-p+j=m} \Phi_{p q j}\right] \oplus$ $\left[\bigoplus_{q-p+j-1=m}^{q-p+n} \Psi_{p q j}\right]$. Then for each $j, \Re^{j}=\bigoplus_{m=-\infty}^{\infty} \mathcal{B}^{j}(m)$, and $S_{1}$ acts on $\mathfrak{B}^{j}(m)$ via the representation $\rho(m)$. Moreover, since $\bar{\partial}_{b}\left(\mathfrak{B}^{j}(m)\right) \subset \mathfrak{B}^{j+1}(m)$, for each $m$ we have a subcomplex

$$
0 \rightarrow \mathfrak{B}^{0}(m) \stackrel{\bar{\partial}_{b}}{\longrightarrow} \mathfrak{B}^{1}(m) \stackrel{\bar{\partial}_{b}}{\longrightarrow} \cdots \stackrel{\bar{\partial}_{b}}{\longrightarrow} \mathfrak{B}^{n-1}(m) \rightarrow 0 .
$$

We now investigate the $\bar{\partial}$ complex on certain holomorphic line bundles on $\mathrm{C} P^{n-1}$. The projection $S_{n} \rightarrow \mathrm{C} P^{n-1}$ exhibits $S_{n}$ as a principal bundle over $\mathrm{C} P^{n-1}$ with structure group $S_{1}$. (In principal bundles the group action is on the 
right, but since $S_{1}$ is commutative, we can think of the left action of $S_{1}$ on $S_{n}$ as a right action.) Let $\eta^{m}$ be the line bundle on $C P^{n-1}$ as sociated to the principal bundle $S_{n} \rightarrow \mathrm{C} P^{n-1}$ by the action $\rho(m)$ of $S_{1}$ on $\mathbf{C}$. (Note that $\eta^{m}$ is the $m$ th tensor power of $\eta=\eta^{1}$.) There are two simple geometrical interpretations of $\eta$ (cf. Hirzebruch [4, $\$ 4.2]$ ). On the one hand, if we think of $C P^{n-1}$ as the set of lines in $\mathbf{C}^{n}$, then $\eta$ is the line bundle whose fiber over $p$ is the line which $p$ is. On the other hand, from the point of view of algebraic geometry, $\eta^{-1}$ is the hyperplane section bundle on $\mathrm{C} P^{n-1}$.

Let $\lambda^{j}=\bigwedge^{j} \bar{T}^{*} \mathrm{C} P^{n-1}$. Then for each $m$ we have the Dolbeault complex

$$
0 \rightarrow \Gamma\left(\eta^{m}\right) \stackrel{\bar{\partial}}{\rightarrow} \Gamma\left(\eta^{m} \otimes \lambda^{1}\right) \stackrel{\bar{\partial}}{\rightarrow} \cdots \stackrel{\bar{\partial}}{\rightarrow} \Gamma\left(\eta^{m} \otimes \lambda^{n-1}\right) \rightarrow 0
$$

whose cohomology group at the $j$ th point may be identified with $H^{j}\left(\mathbf{C} P^{n-1}, \Theta\left(\eta^{m}\right)\right)$ (cf. Hirzebruch $[4, \$ 15.3]$ ). To relate these complexes on $\mathrm{C} P^{n-1}$ with the $\bar{\partial}_{b}$ complex on $S_{n}$ we use the following general theorem:

Theorem 2. Let $P \stackrel{\pi}{\rightarrow} M$ be a principal bundle over the manifold $M$ with structure group $G$. Let $V$ be a vector space on which $G$ acts on the left by the representation $R_{1}, E=P \times{ }_{G} V$ the associated vector bundle over $M$, and $\widetilde{V}=P \times V$ the trivial bundle over $P$ with fiber $V$. If $F$ is any vector bundle over $M$ (not necessarily associated to $P$ ), then $\pi^{*} F$ is a vector bundle over $P$ on which $G$ acts to the right, say by $R_{2}^{\prime}$; we denote the corresponding left action by $R_{2}$, i.e. $R_{2}(g)=R_{2}^{\prime}\left(g^{-1}\right)$. There is a natural one-to-one correspondence between sections of $E \otimes F$ over $M$ and sections $\sigma$ of $\tilde{V} \otimes \pi^{*} F$ over $P$ satisfying

$$
\sigma(x g)=\left(R_{1} \otimes R_{2}\right)\left(g^{-1}\right)[\sigma(x)] .
$$

Proof. This is merely a matter of disentangling the definitions. First we note that $\widetilde{V}$ is naturally isomorphic to $\pi^{*} E$, so $\widetilde{V} \otimes \pi^{*} F \cong \pi^{*}(E \otimes F)$. The right action of $G$ on $\pi^{*} E \cong \widetilde{V}$ is given by $(x, v) g=\left(x g, R_{1}\left(g^{-1}\right) v\right)$, so the left action of $G$ on $\tilde{V} \otimes \pi^{*} F \simeq \pi^{*}(E \otimes F)$ is $R_{1} \otimes R_{2}$. Next, there is a natural surjection $\pi_{*}: \pi^{*}(E \otimes F) \rightarrow E \otimes F$ which is an isomorphism on fibers and satisfies $\pi_{*\left(x_{g}\right)}=$ $\pi_{*(x)} \circ\left(R_{1} \otimes R_{2}\right)(g)$. Thus if $s \in \Gamma(E \otimes F)$, the corresponding $\sigma \in \Gamma\left(\pi^{*}(E \otimes F)\right)$ is given by $\sigma(x)=\pi_{*(x)}^{-1} s(\pi(x))$, and we have

$$
\sigma(x g)=\pi_{*(x g)}^{-1} s(\pi(x g))=\left(R_{1} \otimes R_{2}\right)\left(g^{-1}\right) \pi_{*(x)}^{-1} s(\pi(x))=\left(R_{1} \otimes R_{2}\right)\left(g^{-1}\right) \sigma(x),
$$

so $\sigma$ satisfies (1). Conversely, given $\sigma$ satisfying (1), define $s$ by $s(\pi(x))=$ $\pi_{*(x)} \sigma(x)$; this is well defined since

$$
\pi_{*(x g)} \sigma(x g)=\pi_{*(x)}\left(R_{1} \otimes R_{2}\right)(g)\left(R_{1} \otimes R_{2}\right)\left(g^{-1}\right) \sigma(x)=\pi_{*(x)} \sigma(x) . \quad \text { Q.E.D. }
$$

In our case, we take $M=\mathrm{C} P^{n-1}, G=S_{1}, P=S_{n}, V=\mathbf{C}, R_{1}=\rho(m), E=$ $\eta^{m}$, and $F=\lambda^{j}$. As we noted above, $\pi^{*} \lambda^{j}$ can be naturally identified with the 
bundle $B^{0 j}$. Thus the correspondence of Lemma 2 gives an injection of $\Gamma\left(\eta^{m} \otimes \lambda^{j}\right)$ into $\Gamma\left(B^{0 j}\right)$ which extends to an injection of its completion $L^{2}\left(\eta^{m} \otimes \lambda^{j}\right)$ with respect to the naturally induced hermitian metric into $B^{j}$. We denote this injection by $T_{j}^{m}$.

Theorem 2. The range of $T_{j}^{m}$ is $\mathfrak{B}^{j}(m)$. The diagram

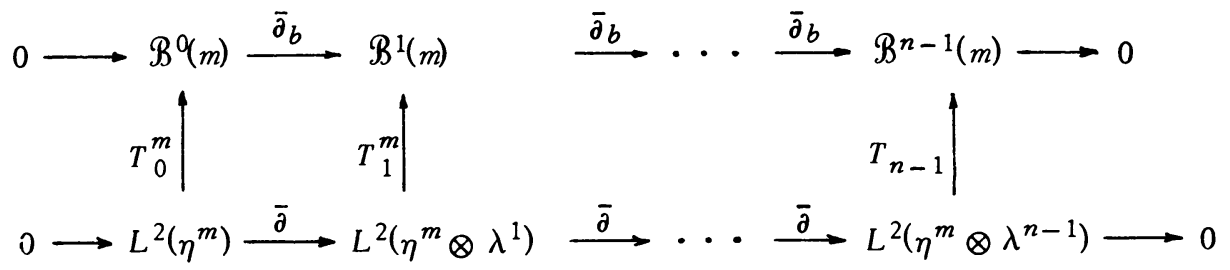

commutes, yielding an isomorphism between the Dolbeault complex of $\eta^{m}$ and the mth piece of the $\bar{\partial}_{b}$ complex on $S_{n}$.

Proof. The only difficulty in proving the first assertion is in keeping the left and right actions straight. If the circle acts to the left on $S_{n}$, there is an induced left action on the antiholomorphic $j$-covectors which we denote by $R_{j}$. This is the natural action we use when considering the $\bar{\partial}_{b}$ complex. But we are considering the action on $S_{n}$ as a right action. Therefore $R_{j}$ becomes a right action, and the corresponding left action required by Lemma 2 is given by $g \rightarrow R_{j}\left(g^{-1}\right)$.

According to these remarks, then, the equivariance conditions for forms in the range of $T_{j}^{m}$ are $\sigma(x g)=\rho_{m}\left(g^{-1}\right) R_{j}(g) \sigma(x)$, which can be written $R_{j}(g) \sigma\left(g^{-1} x\right)=$ $\rho_{m}(g) \sigma(x)$ (since $g^{-1} x=x g^{-1}$ ). But the LHS just defines the action of $S_{1}$ on forms induced by $R_{j}$, so Range $\left(T_{j}^{m}\right)$ is that subspace of $\Re^{j}$ on which $S_{1}$ acts via $\rho(m)$, i.e. Range $\left(T_{j}^{m}\right)=\mathfrak{B}^{j}(m)$.

To prove the second assertion, it suffices to prove the commutativity of the diagram for smooth sections; it then follows easily that $T_{j}^{m}(\operatorname{Dom} \bar{\partial})=\operatorname{Dom} \bar{\partial}_{b}$ and that the diagram commutes in general. Consider the diagram

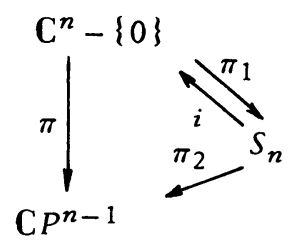

where the maps are the natural injections and projections. Now $\mathbf{C}^{n}-\{0\}$ is a principal bundle over $C P^{n-1}$ with structure group $\mathrm{C}^{*}=\mathrm{C}-\{0\}$, and $S_{n}$ is the corresponding principal bundle with reduced structure group. Thus the consequences of Lemma 2 hold for $\mathrm{C}^{n}-\{0\} \stackrel{\pi}{\rightarrow} \mathrm{C} P^{n-1}$ in a way compatible with those for $S_{n} \stackrel{\pi_{2}}{\rightarrow} \mathrm{C} P^{n-1}$. In particular, to each section $s \in \Gamma\left(\eta^{m} \times \lambda^{j}\right)$ corresponds 
the equivariant $j$-form $\pi_{*(\cdot)} \circ s \circ \pi(\cdot)$ on $\mathrm{C}^{n}-\{0\}$, and $T_{j}^{m}(s)=\pi_{2 *(\cdot)} \circ s \circ \pi_{2}(\cdot)$ $=i *\left(\pi_{*(\cdot)} \circ s \circ \pi(\cdot)\right)$. Replacing $s$ by $\bar{\partial} s$,

$$
\pi_{2 *(\cdot)} \circ \bar{\partial} s \circ \pi_{2}(\cdot)=i *\left(\pi_{*(\cdot)} \circ \bar{\partial} s \circ \pi(\cdot)\right) .
$$

Moreover, if $I(\bar{\partial} r)$ is the ideal generated by $\overline{\partial r}$ ( cf. Chapter I), we have

$$
\pi_{2 *(\cdot)} \circ \bar{\partial} s \circ \pi_{2}(\cdot) \perp I(\bar{\partial} r)
$$

by the remarks at the beginning of this chapter.

Next, since $\pi$ is a holomorphic map, $\pi_{*(\cdot)} \circ \bar{\partial} s \circ \pi(\cdot)=\bar{\partial}\left(\pi_{*(\cdot)} \circ s \circ \pi(\cdot)\right)$. But since $\pi_{*(\cdot)} \circ s \circ \pi(\cdot)$ extends $\pi_{2 *(\cdot)} \circ s \circ \pi_{2}(\cdot)$, it follows from the definition of $\bar{\partial}_{b}$ that $\bar{\partial}_{b}\left(\pi_{2 *(\cdot)} \circ s \circ \pi_{2}(\cdot)\right)$ is determined by the two conditions $\bar{\partial}_{b}\left(\pi_{2 *(\cdot)} \circ s \circ \pi_{2}(\cdot)\right) \equiv i * \bar{\partial}\left(\pi_{*(\cdot)} \circ s \circ \pi(\cdot)\right) \bmod I(\overline{\partial r})$ and $\bar{\partial}_{b}\left(\pi_{2 *(\cdot)} \circ s \circ \pi(\cdot)\right) \perp I(\overline{\partial r})$. Comparing these with (2) and (3), we see that $\bar{\partial}_{b}\left(\pi_{2 *(\cdot)} \circ s \circ \pi_{2}(\cdot)\right)=\pi_{2 *(\cdot)} \circ$ $\bar{\partial} s \circ \pi_{2}(\cdot)$, i.e. $\bar{\partial}_{b} T_{j}^{m} s=T_{j}^{m} \bar{\partial} s$. Q.E.D.

From our knowledge of the $\bar{\partial}_{b}$ complex we can now read off a complete description of the eigenspaces and eigenvalues of the $\bar{\partial}$ complexes on the line bundles $\eta^{m}$. In particular, we have

Theorem 3. If $m \leq 0$,

If $m>0$,

$$
\operatorname{dim} H^{j}\left(\mathbf{C} P^{n-1}, \mathcal{O}\left(\eta^{m}\right)\right)=\left\{\begin{array}{cc}
\left(\begin{array}{c}
n-1-m \\
n-1
\end{array}\right) & (j=0) \\
0 & (j>0)
\end{array}\right.
$$

$$
\operatorname{dim} H^{j}\left(\mathbf{C} P^{n-1}, \mathcal{O}\left(\eta^{m}\right)\right)=\left\{\begin{array}{cc}
0 & (j<n-1), \\
\left(\begin{array}{c}
m-1 \\
n-1
\end{array}\right) & (j=n-1)
\end{array}\right.
$$

where $\left(\begin{array}{c}m-1 \\ n-1\end{array}\right)=0$ if $m<n$.

Proof. The Dolbeault cohomology of $\eta^{m}$ is isomorphic to the $m$ th piece of the $\bar{\partial}_{b}$ cohomology. In particular, it is zero in degrees $1 \leq j \leq n-2$. For $j=0$, the cohomology is

$$
\begin{aligned}
\mathfrak{B}^{0}(m) \cap\left[\bigoplus_{p \geq 0} \Phi_{p 00}\right] & =\left[\begin{array}{cc}
\bigoplus_{q-p=m} \Phi_{p q 0} \cap \underset{p \geq 0}{\bigoplus_{p 00}} \Phi_{p}
\end{array}\right] \\
& =\left\{\begin{array}{cc}
\Phi(-m) 00 & (m \leq 0), \\
0 & (m>0) .
\end{array}\right.
\end{aligned}
$$

But $\Phi_{(-m) 00}$ is the space of homogeneous holomorphic polynomials of degree $-m$ in $n$ variables, whose dimension is $\left(\begin{array}{c}n-1-m \\ n-1\end{array}\right)$. For $j=n-1$, the cohomology is 


$$
\begin{aligned}
\mathfrak{B}^{n-1}(m) \cap & {\left[\bigoplus_{q \geq 1} \Psi_{(-1) q(n-1)}\right] } \\
& =\left[\begin{array}{cc}
\bigoplus_{q-p+n-2=m} \Psi_{p q j}
\end{array}\right] \cap\left[\bigoplus_{q \geq 1} \Psi_{(-1) q(n-1)}\right] \\
& =\left\{\begin{array}{cc}
\Psi_{(-1)(m-n+1)(n-1)} & (m \geq n), \\
0 & (m<n) .
\end{array}\right.
\end{aligned}
$$

But $\Psi_{(-1)(m-n+1)(n-1)}$ is isomorphic (cf. Chapter II, \$4) to the space of homogeneous antiholomorphic polynomials of degree $m-n$ in $n$ variables, and this has dimension $\left(\begin{array}{c}m-1 \\ n-1\end{array}\right)$. Q.E.D.

Of course, this theorem can also be proved directly by the methods of complex analytic geometry. (The reader is invited to perform this computation as a check to our present results.) In fact, if we define $\mathfrak{B}^{j}(m)$ abstractly as the subspace of $\Re^{j}$ transforming under the $S_{1}$ action via $\rho(m)$, Theorem 2 goes through without change; knowing the result of Theorem 3 then enables us to state that the cohomology of the $\bar{\partial}_{b}$ complex is infinite in degrees 0 and $n-1$ and zero elsewhere without knowing the decomposition of the spaces $\mathfrak{B}^{j}$ under the action of $U(n)$.

\section{FOURIER ANALYSIS (REGULARITY OF THE $\bar{\partial}_{b}$ COMPLEX)}

First some definitions and notations:

(1) $S_{(N)}$ denotes the unit sphere in $\mathrm{R}^{n+1}$, so that $S_{(2 n-1)} \cong S_{n}$. (We reserve the notation $S^{N}$ for the $N$-sphere considered abstractly.)

(2) $\mathcal{H}_{k}$ denotes the space of spherical harmonics of degree $k$ on $S_{(N)}$, i.e. the space of homogeneous harmonic polynomials of degree $k$ on $\mathbf{R}^{N+1}$ restricted to $S_{(N)}$.

(3) $\Delta^{*}$ denotes the Laplace-de Rham operator on $S_{(N)}$.

(4) \|\| denotes the $L^{2}$ norm, \|\|$_{s}(s \in \mathbf{R})$ denotes the Sobolev $s$-norm, and \|\|$_{\infty}$ denotes the uniform norm.

(5) If $A$ and $B$ are nonnegative functions of $x, A(x) \sim B(x)$ means that $A(x)=\mathcal{O}(B(x))$ and $B(x)=\mathcal{O}(A(x))$; that is, there exist positive constants $c_{1}, c_{2}$ such that $c_{1} A(x) \leq B(x) \leq c_{2} A(x)$ for all $x$.

(6) $A(x) \lesssim B(x)$ means that $A(x) \leq c B(x)$ for some $c>0$ independent of $x$, i.e. $A(x)=\mathcal{O}(B(x))$.

1. Distribution theory on spheres. One can do distribution theory on $S_{(N)}$ by letting spherical harmonic expansions play the role of Fourier transforms. This is a well-known part of the folk literature of Fourier analysis, but we reproduce the proofs as there seems to be no convenient reference. We will need the following facts (cf., e.g., Müller [13] for proofs); 
(1) $\mathcal{H}_{k_{1}} \perp \mathcal{H}_{k_{2}}$ for $k_{1} \neq k_{2}$, and $L^{2}\left(S_{(N)}\right)=\bigoplus_{0}^{\infty} \mathcal{H}_{k}$.

(2) $\operatorname{dim} \mathcal{H}_{k}=(2 k+N-1)((k+N-2)$ ! /(N-1)! $k$ !).

(3) $\mathcal{H}_{k}$ is an eigenspace of $\Delta^{*}$ with eigenvalue $k(k+N-1)$.

We can define global Sobolev norms for functions by $\|f\|_{s}=\left\|\left(\Delta^{*}+I\right)^{s / 2} f\right\|$ since $\Delta^{*}$ is elliptic and $S_{(N)}$ is compact. Hence if $f=\Sigma_{0}^{\infty} b_{k}, b_{k} \in \mathcal{H}_{k}$, then

$$
\|f\|_{s}^{2}=\sum_{0}^{\infty}[k(k+N-1)+1]^{s}\left\|b_{k}\right\|^{2} \sim \sum_{0}^{\infty}(k+1)^{2 s}\left\|b_{k}\right\|^{2} .
$$

For forms, we may either define $\|u\|_{s}=\left\|\left(\Delta^{*}+I\right)^{s / 2} u\right\|$ as above, or we may take the sum of the $s$-norms of its coefficients with respect to some basis. We shall only be interested in forms coming from the $\bar{\partial}_{b}$ complex $(N=2 n-1)$, and for these we can compute the $s$-norms as follows. Since $\Delta^{*}$ commutes with $U(n)$, the spaces $\Phi_{p q j}$ and $\Psi_{p q j}$ are eigenspaces of $\left(\Delta^{*}+I\right)^{s / 2}$; hence from the first definition, if $u=\Sigma_{p q a}\left(b_{p q a} \phi_{p q j}^{a}+c_{p q a} \psi_{p q j}^{a}\right)$ (in terms of the canonical basis of Chapter IV),

$$
\|u\|_{s}^{2} \sim \sum_{p q a}\left(\left|b_{p q a}\right|^{2}\left\|\phi_{p q j}^{a}\right\|_{s}^{2}+\left|c_{p q a}\right|^{2}\left\|\psi_{p q j}^{a}\right\|_{s}^{2}\right) .
$$

To compute $\left\|\phi_{p q j}^{a}\right\|_{s}$ and $\left\|\psi_{p q j}^{a}\right\|_{s}$, in turn, we use the second definition with the basis $\left\{d \bar{z}_{i_{1}} \wedge \ldots \wedge d \bar{z}_{i_{j}}\right\}$. By Theorem 1, Chapter IV, each coefficient of $\phi_{p q j}^{a}$ is a spherical harmonic of type $(p, q)$; hence $\left\|\phi_{p q j}^{a}\right\|_{s} \sim(p+q+1)^{s}$. The $\psi$ 's are a bit more complicated. Each coefficient of $\psi_{p q j}$ except that of $d \bar{z}_{1} \wedge \ldots \wedge d \bar{z}_{j}$ is a spherical harmonic of type $(p+1, q)$ and the coefficient of $d \bar{z}_{1} \wedge \ldots \wedge d \bar{z}_{j}$ is

$$
\begin{aligned}
\bar{z}_{1}^{q-1} z_{n}^{p} \sum_{j+1}^{n} z_{a} \bar{z}_{a} \\
=\bar{z}_{1}^{q-1} z_{n}^{p}\left[\frac{q+j-1}{p+q+n-1} \sum_{j+1}^{n} z_{a} \bar{z}_{a}-\frac{p+n-j}{p+q+n-1} \sum_{1}^{j} z_{a} \bar{z}_{a}\right] \\
+\frac{p+n-j}{p+q+n-1} \bar{z}_{1}^{q-1} z_{n}^{p}
\end{aligned}
$$

since $\sum_{1}^{n} z_{a} \bar{z}_{a}=1$. This is the sum of a spherical harmonic of type $(p+1, q)$ and one of type $(\mathrm{p}, \mathrm{q}-1)$. Thus the coefficients of $\psi_{p q j}^{a}$ are sums of spherical harmonics of types $(p+1, q)$ and $(p, q-1)$ so $\left\|\psi_{p q j}^{a}\right\|_{s}^{2} \sim(p+q+2)^{2 s}+$ $(p+q)^{2 s} \sim(p+q+1)^{2 s}$. We have proved

Theorem 1. If $u \in \mathfrak{B}^{j}, u=\Sigma_{p q a}\left(b_{p q a} \phi_{p q j}^{a}+c_{p q a} \psi_{p q j}^{a}\right)$, then

$$
\|u\|_{s}^{2} \sim \sum_{p q a}\left[\left|b_{p q a}\right|^{2}(p+q+1)^{2 s}+\left|c_{p q a}\right|^{2}(p+q+1)^{2 s}\right] .
$$


Next we prove the Sobolev lemma for functions on $S_{(N)}$. We state and prove the crucial estimate in a general form which will also be useful later.

Proposition 1. Let $G$ be a compact group, $H$ a closed subgroup, $V$ an irreducible invariant subspace of $L^{2}(G / H)$ under the action of $G, \rho: G \rightarrow$ Aut $(V)$ the representation of $G$ on $V$, and $D=\operatorname{dim} V$. Suppose there is no other subspace of $L^{2}(G / H)$ on which the action of $G$ is equivalent to $\rho$. Then if the measures of $G$ and $G / H$ are normalized to be 1 , sup $\left\{\|f\|_{\infty}: f \in V,\|f\|=1\right\}=D^{1 / 2}$.

Proof. Let $\pi: G \rightarrow G / H$ be the projection, $\pi^{*} V=\left\{\pi^{*} f=f \circ \pi: f \in V\right\}$. Then $\pi^{*} V$ is an irreducible invariant subspace of $L^{2}(G)$ with representation equivalent to $\rho$, consisting of functions which are constant on cosets of $H$, and $\pi^{*}$ is a unitary equivalence of $V$ and $\pi^{*} V$. Since $\rho$ occurs with multiplicity one in the representation of $G$ on $L^{2}(G / H)$, which is the induced representation of the trivial representation of $H$ on $\mathrm{C}$, Frobenius Reciprocity tells us that the trivial representation occurs with multiplicity one in $\rho \mid H$, i.e. there is a unique $f_{1} \in V$ (up to constant multiples) which is invariant under the action of $H$. Take $\left\|f_{1}\right\|=1$ and complete $f_{1}$ to an orthonormal basis $f_{1}, \cdots, f_{D}$ of $V$. With respect to this basis we form the matrix of entry functions of $\rho$,

$$
\rho_{i j}(g)=\int_{G / H} f_{i}(x)\left[\rho(g) f_{j}\right](x) d x=\left(f_{i}, \rho(g) f_{j}\right) .
$$

By the Peter-Weyl theorem (cf. Ste in [14]), $\rho$ occurs with multiplicity $D$ in $L^{2}(G)$, namely on the spaces $W_{i}(i=1, \cdots, D)$ spanned by the columns $\left\{\rho_{k i}\right\}_{k=1}^{D}$ of the matrix $\left(\rho_{i j}\right)$. Next, observe that for $g \in G, b \in H$,

$$
\begin{aligned}
\rho_{11}(g h) & =\left(f_{1}, \rho(g h) f_{1}\right)=\left(f_{1}, \rho(g) \rho(b) f_{1}\right)=\left(f_{1}, \rho(g) f_{1}\right)=\rho_{11}(g), \\
\rho_{11}(b g) & =\left(f_{1}, \rho(b g) f_{1}\right)=\left(f_{1}, \rho(b) \rho(g) f_{1}\right)=\left(\rho\left(b^{-1}\right) f_{1}, \rho(g) f_{1}\right) \\
& =\left(f_{1}, \rho(g) f_{1}\right)=\rho_{11}(g)
\end{aligned}
$$

by the invariance of $f_{1}$. Since $\rho_{11}(g h)=\rho_{11}(g)$ and $\rho$ occurs with multiplicity one in $L^{2}(G / H)$, we must have $\rho_{11} \in \pi^{*} V$; then since $\rho_{11}(b g)=\rho_{11}(g)$, we must have $\rho_{11}=c \pi^{*} f_{1}$ for some constant $c$. In fact, by the Schur orthogonality relations, $\left\|\rho_{11}\right\|=D^{-1 / 2}$ and hence $c=D^{-1 / 2}$. Since an irreducible subspace is specified by giving one vector in it, it follows that $\pi^{*} V=W_{1}$.

Lemma 1. D $\rho_{11}$ is a reproducing kernel for $W_{1}$, i.e. for any $f \in W_{1}, f=$ $D \rho_{11} * f$ where * denotes convolution.

Proof. It suffices to prove the assertion for the basis $\left\{\rho_{i 1}\right\}$. 


$$
\begin{array}{rl}
\rho_{i 1} & * \rho_{11}(x)=\int_{G} \rho_{i 1}\left(x y^{-1}\right) \rho_{11}(y) d y \\
& =\sum_{j} \int_{G} \rho_{i j}(x) \rho_{j 1}\left(y^{-1}\right) \rho_{11}(y) d y \\
& =\sum_{j} \rho_{i j}(x) \int_{G} \overline{\rho_{1 j}(y)} \rho_{11}(y) d y \\
& =D^{-1} \sum_{j} \rho_{i j}(x) \delta_{1 j}=D^{-1} \rho_{i 1}(x)
\end{array}
$$

by Schur orthogonality.

Now by Young's inequality, for any $f \in W_{1},\|f\|_{\infty} \leq D\|f\|\left\|\rho_{11}\right\|=D^{1 / 2}\|f\|$, so $\sup \left\{\|f\|_{\infty}: f \in V,\|f\|=1\right\}=\sup \left\{\|f\|_{\infty}: f \in W_{1},\|f\|=1\right\} \leq D^{1 / 2}$. The supremum $D^{1 / 2}$ is actually achieved, since $\left|\pi^{*} f_{1}(e)\right|=D^{1 / 2} \rho_{11}(e)=D^{1 / 2}$ where $e$ is the identity of G. Q.E.D.

We apply Proposition 1 in the case $G=S O(N+1), H=S O(N), G / H=S_{(N)}$, $V=\mathcal{H}_{k}, \quad D \sim(k+1)^{N-1}$. That the spaces $\mathcal{H}_{k}$ are irreducible and inequivalent for $N>1$ follows from the representation theory of $S O(N)$ (cf. Boerner [1]). (In this case, Lemma 1 is equivalent to the classical Funk-Hecke formula for spherical harmonics.) If $N=1, H_{k}(k>0)$ splits into two one-dimensional representations spanned by $e^{i k \theta}$ and $e^{-i k \theta}$; since these have absolute value 1 , the conclusion of Proposition 1 remains valid.

Lemma 2. If $s>N / 2$ then $\|f\|_{\infty} \leqslant\|f\|_{s}$ for all $f \in \mathcal{C}^{\infty}$.

Proof. Let $f=\Sigma_{0}^{\infty} b_{k}, b_{k} \in \mathcal{H}_{k}$. Then

$$
\begin{aligned}
\|f\|_{\infty} & \leq \sum_{0}^{\infty}\left\|b_{k}\right\|_{\infty} \lesssim \sum_{0}^{\infty}\left\|b_{k}\right\|(k+1)^{(N-1) / 2} \\
& \leq\left(\sum_{0}^{\infty}\left\|b_{k}\right\|^{2}(k+1)^{2 s}\right)^{1 / 2}\left(\sum_{0}^{\infty} k^{N-1-2 s}\right)^{1 / 2} \\
& \sim\|f\|_{s}\left(\sum_{0}^{\infty} k^{N-1-2 s}\right)^{1 / 2}
\end{aligned}
$$

and the sum on the right converges provided $s>N / 2$. (Note: This calculation is entirely analogous to the integration in polar coordinates which proves this lemma in $\mathbf{R}^{n}$, since the "eigenfunctions" for the Laplacian on $\mathbf{R}^{n}$ are distributions whose Fourier transforms are supported on a spherical shell.)

Theorem 2 (Sobolev). $H_{s} \subset \mathcal{C}^{r}$ if $s>r+N / 2$. 
Proof. If $D$ is a differential operator of order at most $r,\|D f\|_{\infty} \leqslant\|D f\|_{s-r} \lesssim$ $\|f\|_{s}$ for $s>r+N / 2$, so sequences of $C^{\infty}$ functions which converge in the $s$-norm converge in the $\mathcal{C}^{r}$ topology. Since $H_{s}$ is the completion of $\mathcal{C}^{\infty}$ with respect to \|\|$_{s}, H_{s}$ is continuously embedded in $C^{r}$. Q.E.D.

Thus we see that $C^{\infty}=\bigcap_{s}$, and the $C^{\infty}$ topology is the same as the topology given by the family of norms $\left\{\|\|_{k}: k \in \mathbf{Z}^{+}\right\}$. Since $S_{(N)}$ is compact, the distributions are just the continuous linear functionals on $\mathcal{C}^{\infty}$, and hence the space of distributions is $\bigcup_{H_{s}}$. Every distribution can thus be expanded in spherical harmonics with coefficients that grow at most polynomially.

The Rellich lemma is trivial in this setup. If $s<s^{\prime}$, the isometry $\left(\Delta^{*}+I\right)^{\left(s-s^{\prime}\right) / 2}: H_{s} \rightarrow H_{s^{\prime}}$, when considered as an operator on $H_{s}$, has eigenvalue $\sim(k+1)^{s-s^{\prime}}$ on $\mathcal{H}_{k}$. Hence it is the norm limit of operators of finite rank and therefore compact.

Finally, we state a criterion for real analyticity.

Theorem 3. $f=\Sigma_{0}^{\infty} b_{k} b_{k}\left(b_{k} \in \mathcal{H}_{k},\left\|b_{k}\right\|=1\right)$ is real analytic if and only if for some $a<1, b_{k}=\mathcal{O}\left(a^{k}\right)$.

Proof. Let $D_{1}, \cdots, D_{M}$ be a set of vector fields which spans the tangent space to $S_{(N)}$ at each point. Since $S_{(N)}$ is compact, it follows from the Hadamard radius-of-convergence formula that global real analyticity is equivalent to the existence of $\delta>0$ such that $\left\|D_{j}^{m} f\right\|_{\infty} \lesssim m ! / \delta^{m}$ for $m=0,1, \cdots$, and $j=1$, $\cdots, M$. In particular, $\left\|\Delta^{* m} f\right\|_{\infty} \lesssim(2 m) ! / \delta^{2 m}$. Now

$$
\Delta^{* m} f=\sum_{0}^{\infty}[k(k+N-1)]^{m} b_{k} b_{k}
$$

where

$$
[k(k+N-1)]^{m} b_{k}=\int_{S_{(N)}}\left(\Delta^{* m} f\right) \bar{b}_{k}
$$

so

$$
\begin{aligned}
\left|b_{k}\right| & \leq[k(k+N-1)]^{-m} \int\left|\left(\Delta^{* m} f\right) \bar{b}_{k}\right| \\
& \leq[k(k+N-1)]^{-m}\left\|\Delta^{* m} f\right\|_{\infty} k^{(N-1) / 2} \\
& \lesssim\left((2 m) ! /(k \delta)^{2 m}\right) k^{(N-1) / 2} \lesssim(2 m / k \delta)^{2 m} k^{(N-1) / 2} .
\end{aligned}
$$

For $k$ sufficiently large we may choose $m$ approximately equal to $k \delta / 4$, whence $\left|b_{k}\right| \lesssim(1 / 2)^{k \delta / 2} k^{(N-1) / 2}$. Setting $a=(1 / 2)^{\delta / 4}<1$, we have $\left|b_{k}\right| \lesssim$ $k^{(N-1) / 2} a^{2 k} \lesssim a^{k}$, since $k^{(N-1) / 2}=\mathcal{O}\left(a^{-k}\right)$.

Conversely, if $b_{k}=\mathcal{O}\left(a^{k}\right)$ with $a<1$, this implies $\left|b_{k}\right| \lesssim k^{(1-3 N) / 2} a^{k / 2}$. Therefore 


$$
\begin{aligned}
\left\|\left(\Delta^{*}\right)^{(m+N) / 2} f\right\| & \lesssim \sum_{0}^{\infty} k^{m+N}\left|b_{k}\right|\left\|b_{k}\right\|_{\infty} \\
& \lesssim \sum_{0}^{\infty} k^{m+n+(1-3 N) / 2+(N-1) / 2} a^{k / 2}=\sum_{0}^{\infty} k^{n} a^{k / 2}
\end{aligned}
$$

Let the largest term in the last sum occur at $k=k_{0}$. By comparing the graphs of $x^{m} a^{x / 2}$ and the step function whose value is $k^{n} a^{k / 2}$ on $[k, k+1]$ when $k<k_{0}$ and $k^{m} a^{k / 2}$ on $[k-1, k]$ when $k>k_{0}$, we see that

$$
\begin{aligned}
\sum_{0}^{\infty} k^{m} a^{k / 2} & \leq \int_{0}^{\infty} x^{m} a^{x / 2} d x+k_{0}^{m} a^{k 0 / 2} \\
& \leq \int_{0}^{\infty} x^{m} a^{x / 2} d x+\max _{[0, \infty)}^{m} a^{x / 2} .
\end{aligned}
$$

Now $\log (1 / a)>0$, so if we set $\delta=(1 / 2) \log (1 / a)$ and make the change of variables $x^{\prime}=\delta x$, we obtain

$$
\int_{0}^{\infty} x^{m} a^{x / 2} d x=\int_{0}^{\infty}\left(\frac{x^{\prime}}{\delta}\right)^{m} e^{-x^{\prime}} d\left(\frac{x^{\prime}}{\delta}\right)=\frac{1}{\delta} \cdot \frac{m !}{\delta^{m}} .
$$

On the other hand,

$$
\max _{[0, \infty)} x^{m} a^{x / 2}=\left(\frac{2 m}{e \log (1 / a)}\right)^{m}=\left(\frac{m}{e}\right)^{m} \frac{1}{\delta^{m}} \leq \frac{m !}{\delta^{m}} .
$$

Thus we have shown

$$
\left\|\left(\Delta^{*}\right)^{(m+N) / 2} f\right\|_{\infty} \lesssim \frac{m !}{\delta^{m}}
$$

Finally, by Theorem 2,

$$
\left\|D_{j}^{m} f\right\|_{\infty} \lesssim\|f\|_{m+N} \sim\left\|\left(\Delta^{*}\right)^{(m+N) / 2} f\right\| \lesssim\left\|\left(\Delta^{*}\right)^{(m+N) / 2} f\right\|_{\infty} \lesssim \frac{m !}{\delta^{m}},
$$

which shows $f$ is real analytic. Q.E.D.

Corollary. If $N=2 n-1, u \in \Re^{j}, u=\Sigma\left(b_{k} \phi_{k}+c_{k} \psi_{k}\right)$ where $\phi_{k} \epsilon$ $\bigoplus_{p+q=k} \Phi_{p q j}, \psi_{k} \in \bigoplus_{p+q=k} \Psi_{p q j},\left\|\phi_{k}\right\|=1$ and $\left\|\psi_{k}\right\|_{=1}=1$, then $u$ is real analytic if and only if, for some $a<1, b_{k}$ and $c_{k}$ are $\Theta\left(a^{k}\right)$.

Proof. As in Theorem 1, write $\psi_{k}=\xi_{k}+\eta_{k}$ where the coefficients of $\xi_{k}$ (respectively $\eta_{k}$ ) are spherical harmonics of degree $k+1$ (respectively $k-1$ ). Then apply Theorem 3 to the coefficient of each $d \bar{z}_{i_{1}} \wedge \cdots \wedge d \bar{z}_{i_{j}}$ in the sums $\Sigma b_{k} \phi_{k}, \Sigma c_{k} \xi_{k}, \Sigma c_{k} \eta_{k}$. (Details are left to the reader.) 
2. The index of $\bar{\partial}_{b}$. As an application of these methods for $N=1$, we compute the index of $\bar{\partial}_{b}$ (considered as an operator on $\bigoplus_{0}^{n-1} \Re^{j}$ ). The kernel and cokernel of an operator which is transversally elliptic with respect to a group $G$ are representation spaces of $G$ and hence have characters which are defined as distributions on $G$. The index is the difference of these characters.

Let $z=e^{i \theta}$ be the standard coordinate on $S_{1}=S_{(1)}$, so the character of $\rho_{k}$ is $z^{k}$. Then by the results of Chapter III,

$$
\operatorname{index}\left(\bar{\partial}_{b}\right)=\sum_{0}^{\infty}\left(\begin{array}{c}
n-1+k \\
n-1
\end{array}\right) z^{k}-\sum_{n+1}^{\infty}\left(\begin{array}{l}
k-1 \\
n-1
\end{array}\right) z^{-k} .
$$

The remarks of $\S_{1}$ show that this is indeed a distribution on $S_{1}$ and in fact belongs to $H_{s}$ for $s<-n$ since $\left(\begin{array}{c}n-1+k \\ n-1\end{array}\right)$ and $\left(\begin{array}{c}k-1 \\ n-1\end{array}\right)$ are $O\left(k^{n-1}\right)$.

Moreover,

$$
\begin{aligned}
& \sum_{0}^{\infty}\left(\begin{array}{c}
n-1+k \\
n-1
\end{array}\right) z^{k}=(1-z)^{-n} \text { for }|z|<1, \\
& \sum_{n+1}^{\infty}\left(\begin{array}{c}
k-1 \\
n-1
\end{array}\right) z^{-k}=(1-z)^{-n} \text { for }|z|>1 .
\end{aligned}
$$

Thus index $\left(\bar{\partial}_{b}\right)$ is the Sato hyperfunction associated with the analytic function $(1-z)^{-n}$ on $\mathrm{C}-S_{1}$, in accordance with a general formula of Atiyah (not yet published).

3. Global regularity of $\bar{\partial}_{b}$. The global regularity properties of the $\bar{\partial}_{b}$ complex can be easily read off by looking at the eigenvalues of $\bar{\partial}_{b}$ vis-à-vis the results of $\$ 1$. Kohn and Nirenberg [8] have shown that corresponding local regularity properties hold on $\mathfrak{B}^{j}$ for $1 \leq j \leq n-2$, but these results seem to be inaccessible by our present methods.

Combining Theorem 1 with the fact that $\gamma_{p q j} \sim((p+1) q)^{1 / 2}$ and the inequality $p+q+1 \leq 2(p+1) q \leq(p+q+1)^{2}$ (except when $p=-1$ or $q=0$ ) which is the sharpest possible inequality relating $(p+1) q$ with powers of $p+q+1$, we obtain the following results, which we state as a theorem:

Theorem 4. (1) For $1 \leq j \leq n-2$, the equation $\square_{b} u=v$ bas a unique solution for every distribution-valued form $v$. The estimate $\|u\|_{s-t} \leqslant\left\|\square \square_{b}^{t} u\right\|_{s-2 t} \leqslant\|u\|_{s}$ bolds whenever $u \in H_{s}$ and $t \geq 0$, and this inequality is the sharpest possible. In particular, taking $t=1$, we see that the application of $\square_{b}$ results in the loss of between one and two derivatives, the exact amount depending on the "spectrum" of the form in question. For forms $u \in \bigoplus_{q=1}^{\infty}\left(\Phi_{p q j} \oplus \Psi_{p q j}\right)(p=$ const. $)$ or $u \in \bigoplus_{p=0}^{\infty}\left(\Phi_{p q j} \oplus \Psi_{p q j}\right) \quad(q=$ const. $),\left\|\square_{b} u\right\|_{s} \sim\|u\|_{s+1} ;$ for forms $u \in$ 
$\bigoplus_{p-q=\text { const. }}\left(\Phi_{p q j} \oplus \Psi_{p q j}\right),\left\|\square_{b} u\right\|_{s} \sim\|u\|_{s+2^{\circ}}$ (Similar remarks apply in the following cases.)

(2) On $\mathfrak{B}^{0}$ and $\mathfrak{B}^{n-1}$ the barmonic space $\mathcal{H}$ is infinite-dimensional, so we cannot bope to obtain any regularity for arbitrary solutions of $\square_{b} u=v$. However, if $v \perp \mathcal{H}$, then there is a unique solution $u \perp \mathcal{H}$ of $\square_{b} u=v$, and $\|u\|_{s+1} \lesssim\|v\|_{s}$ $\lesssim\|u\|_{s+2}$.

(3) If $v \in H_{s}\left(\Re^{j}\right)(0 \leq j \leq n-1), \bar{\partial}_{b} v=0$ and $v$ is orthogonal to the barmonic space (if any), then there is a unique solution $u$ of $\bar{\partial}_{b} u=v$ which is orthogonal to the null space of $\bar{\partial}_{b}$, and $\|u\|_{s+1 / 2} \leqslant\|v\|_{s} \leqslant\|u\|_{s+1}$. Likewise for $\delta_{b}$.

(4) In all of the above cases, if $v$ is real analytic, then the solution $u$ will also be real analytic, since exponential decrease of the coefficients is not affected by factors like $((p+1) q)^{1 / 2}$ or $(p+1) q$.

Details of the proofs are left as a (trivial) exercise.

4. The $b$-norms. In this section we introduce Sobolev-type norms for $\square_{b}$. We shall work exclusively with functions; analogous results for forms are obtained by taking norms componentwise. We could define a norm by $\|f\|=\left\|\left(\square_{b}+I\right) f\right\|$, but this is not very satisfactory because $\square_{b}$ is lopsided: it has a large null space for which this norm provides no information. We proceed to remedy the defect.

Let $\bar{\square}_{b}$ be the conjugate operator to $\square_{b}$, defined by $\bar{\square}_{b} f=\left(\bar{\square}_{b} \overline{\bar{f}}\right)$. Then the $\Phi_{p q 0}$ 's are eigenspaces for $\bar{\square}_{b}$ with eigenvalue $\gamma_{q p 0}^{2}$. Thus $\square_{b}+\bar{\square}_{b}$ is symmetric with respect to $p$ and $q$ and annihilates only constants; the eigenvalue of $\square_{b}+\bar{\square}_{b}+I$ on $\Phi_{p q 0}$ is $\gamma_{p q 0}^{2}+\gamma_{q p 0}^{2}+1 \sim(p+1)(q+1)$. We therefore define the family of Sobolev-type norms $\left\{\|\|_{s, 0}: s \in \mathbf{R}\right\}$ by $\|f\|_{s, 0}=$ $\left\|\left(\square_{b}+\bar{\square}_{b}+I\right)^{s / 2} f\right\|$. (The subscript zero is included to facilitate a later generalization.) The results of $\$ 3$ show that \|\|$_{s / 2} \leqslant\|\|_{s, 0} \leqslant\|\|_{s}$ for $s \geq 0$ and \|\|$_{s} \lesssim\|\|_{s, 0} \leqslant\|\|_{s / 2}$ for $s<0$; more precisely, \|\|$_{s, 0} \sim\|\|_{s / 2}$ on $\bigoplus_{p=\text { const. }} \Phi_{p q 0}$ and $\bigoplus_{q=\text { const. }} \Phi_{p q 0}$ and \|\|$_{s, 0} \sim\|\|_{s}$ on $\bigoplus_{p-q=\text { const. }} \Phi_{p q 0}$. We denote the completion of $\bigcup^{\infty}$ with respect to $\|\mid\| \|_{s, 0}$ by $B_{s, 0}$. The distribution theory of $\oint_{1}$ can then be reformulated in terms of the spaces $B_{s, 0}$. Specifically, we have $C^{\infty}=\bigcap_{B_{s, 0}}$; every distribution belongs to some $B_{s, 0} ;\|\|_{s, 0}$ is compact with respect to \|\|$\|_{s^{\prime}, 0}$ whenever $s>s^{\prime}$; and $B_{s, 0} \subset \mathcal{C}^{s}$ whenever $s>(2 n-1)+2 r$.

$\square_{b}$ and $\bar{\square}_{b}$ are only half as strong in the direction tangent to the circle orbits as in the other directions (cf. Chapter I), and we can therefore obtain sharper estimates if we can control differentiation in the "bad" direction directly. Fortunately, the unit vector field $X$ tangent to the circle orbits is just the infinitesimal generator of the circle action. Since $S_{1}$ is the center of $U(n), X$ commutes with the action of $U(n)$, and so the $\Phi_{p q} 0$ 's are eigenspaces of $X$. 
Proposition 2. The eigenvalue of $X$ on $\Phi_{p q 0}$ is $i(p-q)$.

Proof. According to Theorem 1, Chapter III, every $f \in \Phi_{p q 0}$ satisfies $f\left(e^{-i \theta} x\right)=e^{i(q-p) \theta} f(x)$. Therefore $X f(x)=d / d \theta\left[f\left(e^{i \theta} x\right)\right]_{\theta=0}=i(p-q) f(x)$. Q.E.D.

Letting $|X|=\left(-X^{2}\right)^{1 / 2}$ be the operator whose eigenvalue on $\Phi_{p q 0}$ is $|p-q|$, we define the norms || $\mid \|_{s, \sigma}(s, \sigma \in \mathbf{R})$ by

$$
\|f\|_{s, \sigma}=\left\|\left(\square_{b}+\bar{\square}_{b}+I\right)^{s / 2}(|X|+I)^{\sigma} f\right\|,
$$

and we let $B_{s, \sigma}$ be the completion of $\mathcal{C}^{\infty}$ with respect to \|\|$_{s, \sigma}$.

Proposition 3. For $\sigma \geq 0$,

$$
\|\|\left\|_{s, 0} \lesssim\right\|\|\|_{s, \sigma} \lesssim\|\|_{s+2 \sigma, 0^{\circ}}
$$

Proof. The first inequality is obvious. On the other hand, let $f=\Sigma f_{p q} b_{p q}$ where $f_{p, q} \in \mathbf{C}, b_{p q} \in \Phi_{p q 0},\left\|b_{p q}\right\|=1$. Then

$$
\begin{aligned}
\|f \mid\|_{s, \sigma}^{2} & \sim \sum(p+1)^{s}(q+1)^{s}(|p-q|+1)^{2 \sigma}\left|f_{p q}\right|^{2} \\
& \lesssim \sum[(p+1)(q+1)]^{s+2 \sigma}\left|f_{p q}\right|^{2} \sim\|f\|_{s+2 \sigma, 0}^{2}
\end{aligned}
$$

Moreover, these inequalities are the sharpest possible: \|\|$\left\|_{s, 0} \sim\right\|\|\|_{s, \sigma}$ on $\bigoplus_{p-q=\text { const. }} \Phi_{p q 0}$ and $\left|\|\mid\|_{s+2 \sigma, 0} \sim\|\| \|_{s, \sigma}\right.$ on $\bigoplus_{p=\text { const. }} \Phi_{p q 0}$ and $\bigoplus_{q=\text { const. }} \Phi_{p q 0^{\circ}}$ Q.E.D.

Theorem 5. If $s, \sigma \geq 0$, then \|\|$_{s^{\prime}} \lesssim\|\|_{s, \sigma} \leqslant\|\|_{s+\sigma}$ where $s^{\prime}=$ $\min (s,(s / 2)+\sigma)$. These inequalities are sharp.

Proof. Let $f=\Sigma f_{p q} b_{p q}$ as above. Our method will be to break up sums of the form $\Sigma_{p, q \geq 0} A(p, q)$ into $\Sigma_{p<q}+\Sigma_{p=q}+\Sigma_{p>q}$ and then, setting $m=|p-q|$, rewrite this as

$$
\sum_{m>0, p \geq 0} A(p, p+m)+\sum_{p \geq 0} A(p, p)+\sum_{m>0, q \geq 0} A(q+m, q) .
$$

The idea behind this is to change from the coordinates $(p, q)$ in "Fourier transform space" to the coordinates $(p+q, p-q)$, in which the directions parallel to the axes are the directions of greatest strength of $\square_{b}+\bar{\square}_{b}$ and $X$, respectively.

First, suppose $\sigma \geq s / 2$, so $\min (s,(s / 2)+\sigma)=(s / 2)+\sigma$. Then

$$
\begin{aligned}
\|f\|_{(s / 2)+\sigma}^{2} & \sim \sum_{p, q \geq 0}(p+q+1)^{s+2 \sigma}\left|f_{p q}\right|^{2} \\
& \sim \sum_{m>0, p \geq 0}(2 p+m+1)^{s+2 \sigma}\left|f_{p(p+m)}\right|^{2}+\sum_{p \geq 0}(2 p+1)^{s+2 \sigma}\left|f_{p p}\right|^{2} \\
& +\sum_{m>0, q \geq 0}(2 q+m+1)^{s+2 \sigma}\left|f_{(q+m) q}\right|^{2}
\end{aligned}
$$


Now

$$
\begin{aligned}
\sum_{m>0, p \geq 0} & (2 p+m+1)^{s+2 \sigma}\left|f_{p(p+m)}\right|^{2} \\
& \lesssim \sum_{m>0, p \geq 0}(p+m+1)^{s}(p+m+2)^{2 \sigma}\left|f_{p(p+m)}\right|^{2} \\
& \lesssim \sum_{m>0, p \geq 0}(p+m+1)^{s}(p+1)^{2 \sigma}(m+1)^{2 \sigma}\left|f_{p(p+m)}\right|^{2} \\
& \lesssim \sum_{m>0, p \geq 0}(p+m+1)^{s}(p+1)^{s}(m+1)^{2 \sigma}\left|f_{p(p+m)}\right|^{2} \\
& =\sum_{p<q}(p+1)^{s}(q+1)^{s}(|p-q|+1)^{2 \sigma}\left|f_{p q}\right|^{2},
\end{aligned}
$$

and likewise

$$
\sum_{m>0, q \geq 0}(2 q+m+1)^{s+2 \sigma}\left|f_{(q+m) q}\right|^{2} \leqslant \sum_{p>q}(p+1)^{s}(q+1)^{s}(|p-q|+1)^{2 \sigma}\left|f_{p q}\right|^{2}
$$

Also,

$$
\sum_{p \geq 0}(2 p+1)^{s+2 \sigma}\left|f_{p p}\right|^{2} \lesssim \sum_{p \geq 0}(p+1)^{s+2 \sigma}\left|f_{p p}\right|^{2} \lesssim \sum_{p \geq 0}(p+1)^{2 s}\left|f_{p p}\right|^{2}
$$

Therefore

$$
\|f\|_{(s / 2)+\sigma}^{2} \lesssim \sum_{p, q \geq 0}(p+1)^{s}(q+1)^{s}(|p-q|+1)^{2 \sigma}\left|f_{p q}\right|^{2} \sim\|f\|_{s, \sigma}^{2} .
$$

An examination of this calculation shows that \|\|$_{(s / 2)+\sigma} \sim\|\| \|_{s, \sigma}$ on $\bigoplus_{p=\text { const. }} \Phi_{p q 0}$ and $\bigoplus_{q=\text { const. }} \Phi_{p q 0}$.

Next, suppose $\sigma \geq s / 2$, so $\min (s,(s / 2)+\sigma)=s$. Then essentially the same calculation with the roles of $s$ and $2 \sigma$ reversed shows that \|\|$_{s} \leqslant\|\| \|_{s, \sigma}$ and \|\|$_{s} \sim\|\|_{s, \sigma}$ on $\bigoplus_{p-q=\text { const. }} \Phi_{p q 0}$. Finally,

$$
\begin{aligned}
\|f\|_{s, \sigma}^{2} & \sim \sum_{p, q \geq 0}(p+1)^{s}(q+1)^{s}(|p-q|+1)^{2 \sigma}\left|f_{p q}\right|^{2} \\
& \lesssim \sum_{p, q \geq 0}(p+q+1)^{2 s+2 \sigma}\left|f_{p q}\right|^{2} \sim\|f\|_{s+\sigma}^{2},
\end{aligned}
$$

and \|\|$\left\|_{s, \sigma} \sim\right\| \|_{s+\sigma}$ on (for example) $\bigoplus_{p \geq 0} \Phi_{p(2 p) 0}$ Q.E.D.

Corollaries. (1) For $s, \sigma \geq 0$ we have $B_{s+2 \sigma, 0} \subset B_{s, \sigma} \subset B_{s, 0}$ and $H_{s+\sigma} C$ $B_{s, \sigma} \subset H_{\min (s,(s / 2)+\sigma)}$. These inclusions are sharp and are continuous but not compact. 
(2) $B_{s, \sigma}$ is naturally dual to $B_{-s,-\sigma}$, so the the inclusion relations for $s, \sigma$ $<0$ are obtained by dualizing those in (1).

(3) $\mathcal{C}^{\infty}=\bigcap_{B_{s, \sigma}}$, and every distribution lies in some $B_{s, \sigma}$.

(4) || $\mid \|_{s, \sigma}$ is compact with respect to \|\|$\|_{s^{\prime}, \sigma^{\prime}}$ if and only if $s>s^{\prime}$ and $\sigma \geq \sigma^{\prime}$.

Thus we can do distribution theory with the spaces $B_{s, \sigma}$, and we get more efficient relations with the $H_{s}$ spaces than with the spaces $B_{s, 0}$. The analogue of the Sobolev theorem is that $B_{s, \sigma} \subset \mathcal{C}^{r}$ if $s>r+n-1 / 2$ and $\sigma \geq(r+n-1 / 2) / 2$; we can also take $\sigma$ to be smaller if we let $s$ be larger. However, we can obtain a much sharper result for $r=0$ by a direct argument.

Lemma 1. If $b_{p q} \in \Phi_{p q 0}$ and $\left\|b_{p q}\right\|=1$, then

$$
\left\|b_{p q}\right\|_{\infty} \lesssim(p+1)^{(n-2) / 2}(q+1)^{(n-2) / 2}(p+q+1)^{1 / 2} \text {. }
$$

Proof. By the general formula for the dimensions of representations of $U(n)$ (Boerner [1, p. 201]), we have

$$
\operatorname{dim} \Phi_{p q 0}=\frac{(p+n-2) !(q+n-2) !(p+q+n-1)}{p ! q !(n-1) !(n-2) !} \sim(p+1)^{n-2}(q+1)^{n-2}(p+q+1) .
$$

The lemma now follows from Proposition 1, taking $G=U(n), H=U(n-1)$. Q.E.D.

Theorem 6. $B_{s, \sigma} \subset \mathcal{C}^{0}$ if for some $\epsilon>0, s>n-1+\epsilon$ and $\sigma>(1-\epsilon) / 2$, and the inclusion is continuous.

Proof. Let $f=\Sigma f_{p q} b_{p q}$ as above. By Lemma 1,

$$
\begin{aligned}
\|f\|_{\infty} \leq & \sum\left|f_{p q}\right|\left\|b_{p q}\right\|_{\infty} \\
\leq & \sum\left|f_{p q}\right|(p+1)^{(n-2) / 2}(q+1)^{(n-2) / 2}(p+q+1)^{1 / 2} \\
& \cdot(p+1)^{s / 2}(q+1)^{s / 2}(|p-q|+1)^{\sigma} /(p+1)^{s / 2}(q+1)^{s / 2}(|p-q|+1)^{\sigma} \\
\leq & {\left[\sum(p+1)^{s}(q+1)^{s}(|p-q|+1)^{2 \sigma}\left|f_{p q}\right|^{2}\right]^{1 / 2} } \\
& \cdot\left[\sum(p+1)^{n-2-s}(q+1)^{n-2-s}(p+q+1)(|p-q|+1)^{-2 \sigma}\right]^{1 / 2}
\end{aligned}
$$

by the Schwarz inequality. The first factor in the last expression is $\sim\|f\| \|_{s, \sigma}$; it remains to show that the second factor converges. Using the same trick as in Theorem 5,

$$
\begin{aligned}
\sum_{p, q \geq 0} & {[(p+1)(q+1)]^{n-2-s}(p+q+1)(|p-q|+1)^{-2 \sigma} } \\
= & \sum_{m>0, p \geq 0}[(p+1)(p+m+1)]^{n-2-s}(2 p+m+1)(m+1)^{-2 \sigma}+\sum_{p \geq 0}(p+1)^{2(n-2-s)}(2 p+1) \\
& +\sum_{m>0, q \geq 0}[(q+m+1)(q+1)]^{n-2-s}(2 q+m+1)(m+1)^{-2 \sigma} .
\end{aligned}
$$


The second term is less than $2 \Sigma_{p \geq 0}(p+1)^{2 n-2 s-3}$, which converges provided $2 n-2 s-3<-1$, i.e. provided $s>n-1$. The first and third terms are equal, and we have, assuming $s>n-1$,

$$
\begin{aligned}
\sum_{m>0, p \geq 0}[ & (p+1)(p+m+1)]^{n-2-s}(2 p+m+1)(m+1)^{-2 \sigma} \\
& \leqslant \sum_{m>0}(m+1)^{-2 \sigma} \sum_{p \geq 0}(p+1)^{n-2-s}(p+m+1)^{n-1-s} \\
& =\sum_{m>0}(m+1)^{n-1-s-2 \sigma} \sum_{p \geq 0}(p+1)^{n-2-s}(p /(m+1)+1)^{n-1-s} \\
& \leq \sum_{m>0}(m+1)^{n-1-s-2 \sigma} \sum_{p \geq 0}(p+1)^{n-2-s}
\end{aligned}
$$

since $(p /(m+1)+1)^{n-1-s} \leq 1$. Convergence of the second factor again requires $s>n-1$. Convergence of the first factor requires $n-1-s-2 \sigma\langle-1$, i.e. $\sigma\rangle$ $(1+(n-1-s)) / 2$. Taking $\epsilon=(s-n+1) / 2$, we see that under the hypotheses of the theorem, \|\|$_{\infty} \leqslant\|\|_{s, \sigma}$, and the conclusion follows immediately. Q.E.D.

Corollary. $B_{s, 0} \subset \mathcal{C}^{0}$ if $s>n$.

This follows directly from Theorem 6 and Proposition 3.

We conclude with some heuristic remarks. Theorem 6 says (except for the factor of $\epsilon$ ) that $B_{s, \sigma} \subset \mathcal{C}^{0}$ if $s>n-1$ and $\sigma>1 / 2$. The results of Chapter III show that $\square_{b}$ is essentially the pullback of the Laplacian on $C P^{n-1}$, and $X^{2}$ restricted to an orbit is just the Laplacian on $S_{1}$. The ordinary Sobolev theorem says that $H_{s}\left(\mathbf{C} P^{n-1}\right) \subset \mathcal{C}^{0}\left(\mathbf{C} P^{n-1}\right)$ if $s>n-1$ and $H_{\sigma}\left(S_{1}\right) \subset \mathcal{C}^{0}\left(s_{1}\right)$ if $\sigma>1 / 2$. Thus, in some sense, these two phenomena are combined by the fibration $S_{1} \rightarrow S_{n} \rightarrow \mathrm{CP} P^{n-1}$ to yield our result.

It seems likely that the following generalization of Theorem 6 should be true:

Conjecture. $B_{s, \sigma} \subset \mathcal{C}^{r}$ if for some $\epsilon>0, s>r+n-1+\epsilon$ and $\sigma>(r+1-\epsilon) / 2$.

The estimate needed to establish this assertion is $\|D f\|_{s, \sigma} \leqslant\|f\|_{s+r, \sigma_{+} / 2}$ where $D$ is any differential operator of order at most $r$. Indeed, we have

$$
\begin{aligned}
\left\|X^{j} f\right\|_{s, \sigma} & \sim \sum(p+1)^{s}(q+1)^{s}(|p-q|+1)^{2 \sigma}|p-q|^{2 j}\left|f_{p q}\right|^{2} \\
& \leq \sum(p+1)^{s+j}(q+1)^{s+j}(|p-q|+1)^{2 \sigma+j}\left|f_{p q}\right|^{2} \\
& \sim\|f \mid\|_{s+j, \sigma+j / 2^{.}}
\end{aligned}
$$

Hence it suffices to prove the estimate for $D$ involving only differentiations in directions orthogonal to $X$. But $\square_{b}$ is "elliptic" in these directions, in a sense made precise by Theorem 2 of Chapter III, so there should be good control over such $D$ 's. However, we have not yet found a satisfyingly rigorous proof. 


\section{BESSEL FUNCTIONS \\ (THE $\bar{\partial}$-NEUMANN PROBLEM ON THE UNIT BALL)}

Let $\mathbb{Q}^{j}$ denote the Hilbert space of square-integrable $(0, j)$ forms on the unit ball $B_{n} \subset \mathrm{C}^{n}$, so that $\mathbb{Q}^{j}$ is the completion of $\Gamma\left(\underline{A}^{0 j}\right)$ in the notation of Chapter $\mathrm{I}$. Recall that $u \in \Gamma\left(\underline{A}^{j}\right)$ is said to satisfy the $\bar{\partial}$-Neumann conditions if $u \mid S_{n} \in B^{j}$ and $\bar{\partial} u \mid S_{n} \in B^{j+1}$. The restriction of $\bar{\partial} \delta+\delta \bar{\partial}$ to forms satisfying the $\bar{\partial}$-Neumann conditions is a positive hermitian operator; we denote its Friedrichs extens ion by $\square$ (and use the symbol $\square$ only for this purpose). In this chapter we will solve the following strong form of the $\bar{\partial}$-Neumann problem on $B_{n}$ : determine the spectral decomposition of $\mathbb{Q}^{j}$ under $\square$, that is, find the eigenvectors and eigenvalues for $\square$. Actually, in order not to clutter up the notation with factors of 2 , we will deal with the operator $2 \square$, which as a differential operator is just the Laplace-de Rham operator.

1. The $\bar{\partial}$-Neumann problem for functions. For functions we have the wellknown formula

$$
2 \square=-\frac{\partial^{2}}{\partial r^{2}}-\frac{2 n-1}{r} \frac{\partial}{\partial r}+\frac{1}{r^{2}} \Delta^{*}
$$

(cf. Müller [13, p. 38]) where $\Delta^{*}$ is the Laplacian on $S_{n}$. Since the radial and tangential differentiations are not mixed, the trick of "separation of variables" works, and the eigenfunctions will be of the form $f(r) g(\theta)$, where $\theta$ denotes a coordinate on $S_{n}$. But we already know that functions on $S_{n}$ decompose under $\Delta^{*}$ into spherical harmonics; therefore, adjusting a factor of $r^{p+q}$, we seek eigenfunctions of the form $f(r) b_{p q}$ where $b_{p q}$ is a harmonic polynomial of type $(p, q)$.

First let us see what the $\bar{\partial}$-Neumann conditions mean for such functions. The first condition is vacuous, and the second says $\left.\left\langle\bar{\partial}\left(f(r) b_{p q}\right), \overline{\partial r}\right\rangle\right|_{r=1}=0$. Therefore

$$
\begin{aligned}
0 & =\left.\sum_{1}^{n} \frac{\bar{z}_{a}}{2 r} \frac{\partial}{\partial \bar{z}_{a}}\left(f(r) b_{p q}\right)\right|_{r=1} \\
& =\sum_{1}^{n} \frac{\bar{z}_{a}}{2 r}\left[f^{\prime}(r) \frac{z_{a}}{2 r} b_{p q}+f(r) \frac{\partial b_{p q}}{\partial \bar{z}_{a}}\right]_{r=1}=\left.\frac{1}{2 r}\left[\frac{r f^{\prime}(r)}{2}+q f(r)\right] b_{p q}\right|_{r=1}
\end{aligned}
$$

by the Euler homogeneity formula. Thus the boundary condition is

$$
1 / 2 f^{\prime}(1)+q f(1)=0 \text {. }
$$

With this we quickly dispose of the eigenvalue 0 . Since $b_{p q}$ is already harmonic, by uniqueness for the Dirichlet problem, we must have $f(r)=$ const. (1) then becomes $q=0$, so the null space of $2 \square$ consists precisely of the holomorphic functions. 
The nonzero eigenvalues are all positive, so we may write them as $\lambda^{2}, 0<$ $\lambda<\infty$. To solve the equation $\left(2 \square-\lambda^{2}\right)\left(f(r) b_{p q}\right)=0$, we use the formula

$$
\square(F G)=(\square F) G+F(\square G)-\sum_{1}^{2 n} \frac{\partial F}{\partial x_{a}} \frac{\partial G}{\partial x_{a}}
$$

(where $x_{1}, \cdots, x_{2 n}$ are real Cartesian coordinates on $\mathbf{C}^{n}$ ) and the Euler homogeneity rule, which yield

$$
2 \square\left(f(r) b_{p q}\right)=-\left[f^{\prime \prime}(r)+((2 n+2 p+2 q-1) / r) f^{\prime}(r)\right] b_{p q} .
$$

The equation

$$
f^{\prime \prime}(r)+((2 n+2 p+2 q-1) / r) f^{\prime}(r)+\lambda^{2} f(r)=0
$$

becomes Bessel's equation of order $p+q+n-1$ after the changes of variables $R=\lambda r, \quad F(R)=R^{p+q+n-1} f(R)$, and hence the solutions which are regular at the origin are constant multiples of $r^{1-n-p-q} J_{p+q+n-1}(\lambda r)$.

A short computation shows that the boundary condition (1) is equivalent to

$$
\lambda J_{p+q+n-1}^{\prime}(\lambda)+(q-p-n+1) J_{p+q+n-1}(\lambda)=0 .
$$

It is known from the theory of Bessel functions ([17, Chapter XVIII]) that the positive $\lambda$ 's satisfying this equation form a countable discrete set and that the corresponding functions $J_{p+q+n-1}(\lambda r)$ form a complete orthogonal system with respect to the weight function $r$ on $(0,1)$. (The case $q=0$ is exceptional: here one must add the function $r^{p+n-1}$ to make the system complete, which accounts for the eigenvalue 0 .) The expansion of a function on $(0,1)$ with respect to such a system is called a Dini series.

Let $\lambda_{p q 1}^{01}, \lambda_{p q 2}^{01}, \cdots$ be the countable set of positive $\lambda$ 's satisfying (2) enumerated in increasing order. (The superscript 01 , superfluous at present, will become significant in the next section.) Let

$$
f_{p q m}^{01}(r)=c_{p q} r^{1-n-p-q} J_{p+q+n-1}\left(\lambda_{p q m}^{01} r\right)
$$

where the constant $c_{p q}$ is determined so that $f_{p q m}^{01}(r) b_{p q}$ has $L^{2}$-norm 1 wherever $b_{p q} \mid S_{n}$ has $L^{2}$-norm 1 on $S_{n}$. Moreover, let $b_{p q}^{1}, b_{p q}^{2}, \cdots$ be a complete set of harmonic polynomials of type $(p, q)$ which are orthonormal on $S_{n}$. (For example, we could take $b_{p q}^{a}$ to be the harmonic extension to $B_{n}$ of $\phi_{p q 0}^{a}$ on $S_{n}$.) We then have the solution to the $\bar{\partial}$-Neumann problem for functions:

Theorem 1. The set $\left\{b_{p 0}^{a}\right\}_{p a} \cup\left\{f_{p q m}^{01}(r) b_{p q}^{a}\right\}_{p q m a}$ is an orthonormal basis for $\mathbb{Q}^{0}$ consisting of eigenfunctions for $2 \square$. The eigenvalue of $b_{p 0}^{a}$ is 0 , and the eigenvalue of $f_{p q m}^{01}(r) b_{p q}^{a}$ is $\left(\lambda_{p q m}^{01}\right)^{2}$.

Proof. The orthogonality and completeness follow in the usual way from the 
orthogonality and completeness of spherical harmonics and Bessel functions on $S_{n}$ and $(0,1)$, respectively. Q.E.D.

2. The $\bar{\partial}$-Neumann problem for forms. Here again the method will be to expand forms as in Chapter II on each spherical shell with coefficients depending on $r$ and then to obtain a Bessel equation for these coefficients. The first step, therefore, is to define extensions of $\phi_{p q j}$ and $\psi_{p q j}$ to the interior of the ball, which we will still denote by $\phi_{p q j}$ and $\psi_{p q j}$.

We use the analytical expression

$$
\phi_{p q j}=\bar{z}_{1}^{q-1} z_{n}^{p} \sum_{i=1}^{j+1}(-1)^{i-1} \bar{z}_{i} d \bar{z}_{1} \wedge \ldots \widehat{d}_{i} \cdots \wedge d \bar{z}_{j+1}
$$

to define $\phi_{p q j}$ on all of $B_{n}$. Further we define

$$
\zeta_{i}=d \bar{z}_{i}-\frac{\left\langle d \bar{z}_{i}, \bar{\partial} r\right\rangle}{\langle\bar{\partial} r, \bar{\partial} r\rangle} \overline{\partial r}=d \bar{z}_{i}-\bar{z}_{i} \sum_{i}^{n} \frac{z_{a}}{r} d \bar{z}_{a}=d \bar{z}_{i}-\bar{z}_{i} \frac{2 \bar{\partial} r}{r}
$$

on $B_{n}-\{0\}$ and then de fine

$$
\psi_{p q j}=r \bar{z}_{1}^{q-1} z_{n}^{p} \zeta_{1} \wedge \cdots \wedge \zeta_{j}
$$

The factor of $r$ is inserted to make $\psi_{p q j}$ homogeneous of degree $p+q$, as $\phi_{p q j}$ is; it also has the effect of making every $\psi_{p q j}$ except $\psi_{(-1) 1(n-1)}$ continuous at 0 . It is clear that these extensions of $\phi_{p q j}$ and $\psi_{p q j}$ preserve the property of being pointwise orthogonal to forms of the type $\theta \wedge \bar{\partial}$. It is also easy to check that

$$
\psi_{p q j}=r \bar{z}_{1}^{q-1} z_{n}^{p} d \bar{z}_{1} \wedge \ldots \wedge d \bar{z}_{j}+(-1)^{j} 2 \phi_{p q(j-1)} \wedge \overline{\partial r}
$$

for $p \geq 0$, and

$$
\psi_{(-1) q(n-1)}=\frac{\bar{z}_{1}^{q-1}}{r} \sum_{i=1}^{n}(-1)^{i+n} \bar{z}_{i} d \bar{z}_{1} \wedge \cdots \widehat{d}_{i} \cdots \wedge d \bar{z}_{n}
$$

Now let $\phi_{p q j}^{a}, \psi_{p q j}^{a}$ be the canonical basis forms of Chapter IV. We extend these forms to the interior just as above by requiring their coefficients to be homogeneous of degree $p+q$.

Except at the origin, every $(0, j)$-form $\theta$ can be expressed as $\theta=\theta_{1}+\theta_{2} \wedge \overline{\partial r}$ where $\theta_{1}$ and $\theta_{2}$ are pointwise orthogonal to the ideal generated by $\overline{\partial r} . \theta_{1}$ and $\theta_{2}$ can then be expanded in terms of the $\phi$ 's and $\psi$ 's with coefficients depending on $r$. We therefore look for eigenvectors of $2 \square$ of the form $f(r) \phi_{p q j}^{a}, f(r) \psi_{p q j}^{a}$, $f(r) \phi_{p q j}^{a} \wedge \bar{\partial} r, f(r) \psi_{p q j}^{a} \wedge \bar{\partial}_{r}$ where in the last two cases the boundary condition 
$f(1)=0$ must be satisfied. The thing that will make this easy is the fact that acts componentwise with respect to the natural basis in $\mathbf{C}^{n}$ :

$$
\square\left(\sum a_{i_{1} \cdots i_{j}} d \bar{z}_{i_{1}} \wedge \cdots \wedge d \bar{z}_{i_{j}}\right)=\sum\left(\square a_{i_{1} \cdots i_{j}}\right) d \bar{z}_{i_{1}} \wedge \cdots \wedge d \bar{z}_{i_{j}} .
$$

(This follows from the Weitzenböck formula, or by direct calculation.)

First let us consider forms of the type

$$
f(r) \phi_{p q j}=f(r) \bar{z}_{1}^{q-1} z_{n}^{p} \sum_{i=1}^{j+1}(-1)^{i-1} \bar{z}_{i} d \bar{z}_{1} \wedge \ldots \widehat{d}_{i} \cdots \wedge d \bar{z}_{j+1} .
$$

Since $q \geq 1$ and $j \leq n-2$, each coefficient of $\phi_{p q j}$ is a harmonic monomial of type $(p, q)$. Therefore, applying the arguments of $\$ 1$ to each coefficient, we see that $\left(2 \square-\lambda^{2}\right)\left(f(r) \phi_{p q j}\right)=0$ if and only if $f(r)=c r^{1-n-p-q} J_{p+q+n-1}(\lambda r)$, or $f(r)$ $=c$ in case $\lambda=0$. Moreover,

$$
\begin{aligned}
& \bar{\partial}\left(f(r) \phi_{p q j}\right) \\
& =f^{\prime}(r) \bar{z}_{1}^{q-1} z_{n}^{p}\left(\sum_{i=1}^{j+1}(-1)^{i-1} \bar{z}_{i} d \bar{z}_{1} \wedge \cdots \widehat{d}_{i} \cdots \wedge d \bar{z}_{j+1}\right) \wedge(-1)^{j} \bar{\partial} r \\
& +(q+j) f(r) \bar{z}_{1}^{q-1} z_{n}^{p} d \bar{z}_{1} \wedge \ldots \wedge d \bar{z}_{j+1} \\
& =(q+j) \frac{f(r)}{r} \psi_{p q(j+1)}+\frac{2(-1)^{j}}{r}\left[\frac{r f^{\prime}(r)}{2}+(q+j) f(r)\right] \phi_{p q j} \wedge \bar{\partial} r .
\end{aligned}
$$

Therefore the $\bar{\partial}$-Neumann condition requires

$$
1 / 2 f^{\prime}(1)+(q+j) f(1)=0 .
$$

Thus the eigenvalue 0 does not occur. As in $\$ 1,(3)$ is equivalent to

$$
\lambda J_{p+q+n-1}^{\prime}(\lambda)+(q-p+2 j-n+1) J_{p+q+n-1}(\lambda)=0
$$

and there is a countable sequence $\lambda_{p q 1}^{j 1}, \lambda_{p q 2}^{j 1}, \ldots$ of positive numbers satisfying this equation, yielding an orthogonal sequence of Bessel functions. Setting

$$
f_{p q m}^{j 1}(r)=c_{p q j} r^{1-n-p-q} J_{p+q+n-1}\left(\lambda_{p q m}^{j 1} r\right)
$$

with the normalizing constant $c_{p q j}$ as before, and then letting the unitary group act, we see that $\left\{f_{p q m}^{j 1}(r) \phi_{p q j}^{a}\right\}_{p q m a}$ is an orthonormal basis for the subspace of $\mathbb{Q}^{j}$ whose elements are in the span of the $\phi^{\prime}$ 's on each sphere.

Furthermore, since $2 \square$ commutes with $\bar{\partial}$, the forms $\bar{\partial}\left(f_{p q m}^{j 1}(r) \phi_{p q j}^{a}\right)$ are an orthogonal set of eigenforms with the same eigenvalues $\left(\lambda_{p q m}^{j 1}\right)^{2}$. (They automatically satisfy the second boundary condition since they are $\bar{\partial}$-closed.) $\mathrm{A}$ straightforward computation shows that $\delta\left(f_{p q \mathrm{~m}}^{j 1}(r) \phi_{p q j}^{a}\right)=0$, so 


$$
\left(\lambda_{p q m}^{j 1}\right)^{2} f_{p q m}^{j 1}(r) \phi_{p q j}^{a}=2 \square\left(f_{p q m}^{j 1}(r) \phi_{p q j}^{a}\right)=2 \delta \bar{\partial}\left(f_{p q m}^{j 1}(r) \phi_{p q j}^{a}\right) \text {. }
$$

It follows then from the fact that $\bar{\partial}$ is a weighted shift operator that $\left\{2^{1 / 2}\left(\lambda_{p q m}^{j 1}\right)^{-1} \bar{\partial}\left(f_{p q m}^{j 1}(r) \phi_{p q j}^{a}\right)\right\}_{p q m a}$ is an orthonormal basis for another large subspace of $\mathcal{Q}^{j+1}$, orthogonal to the one constructed above.

We cannot play the same game with the $\psi$ 's or $\phi \wedge \bar{\partial} r$ 's, because their coefficients with respect to the natural basis are not harmonic. However,

$$
\begin{aligned}
& \psi_{p q j} \wedge \bar{\partial} r \\
& =\left[r \bar{z}_{1}^{q-1} z_{n}^{p} d \bar{z}_{1} \wedge \cdots \wedge d \bar{z}_{j}+2(-1)^{j} \phi_{p q(j-1)} \wedge \bar{\partial} r\right] \wedge \bar{\partial} r \\
& =r \bar{z}_{1}^{q-1} z_{n}^{p} d \bar{z}_{1} \wedge \cdots \wedge d \bar{z}_{j} \wedge\left(\frac{1}{2 r} \sum_{1}^{n} z_{i} d \bar{z}_{i}\right) \\
& =\frac{1}{2} \bar{z}_{1}^{q-1} z_{n}^{p} \sum_{j+1}^{n} z_{i} d \bar{z}_{1} \wedge \cdots \wedge d \bar{z}_{j} \wedge d \bar{z}_{i}
\end{aligned}
$$

for $p \geq 0$, and

$$
\begin{aligned}
& \psi_{(-1) q(n-1)} \wedge \overline{\partial r} \\
& =\frac{1}{r} \bar{z}_{1}^{q-1}\left(\sum_{i=1}^{n}(-1)^{i+n} \bar{z}_{i} d \bar{z}_{1} \wedge \ldots \widehat{d}_{i} \cdots \wedge d \bar{z}_{n}\right) \wedge\left(\frac{1}{2 r} \sum_{1}^{n} z_{i} d \bar{z}_{i}\right) \\
& =\frac{1}{2 r^{2}} \bar{z}_{1}^{q-1} \sum_{1}^{n} z_{i} \bar{z}_{i} d \bar{z}_{1} \wedge \cdots \wedge d \bar{z}_{n} \\
& =1 / 2 \bar{z}_{1}^{q-1} d \bar{z}_{1} \wedge \ldots \wedge d \bar{z}_{n} .
\end{aligned}
$$

Since $j \geq 1$, the coefficients of these forms are harmonic monomials of type $(p+1, q-1)$. Thus we obtain eigenforms of $2 \square$ with eigenvalue $\lambda^{2}$ of the type $f(r) \psi_{p q j} \wedge \overline{\partial r}$ by taking $f(r)=c r^{1-n-p-q} J_{p+q+n-1}(\lambda r)$, and the first boundary condition is $f(1)=0$. (Hence $\lambda=0$, which would require $f(r)=c$, is impossible.) The second boundary condition is vacuous, for

$$
\begin{aligned}
& \bar{\partial}\left(f(r) \psi_{p q j} \wedge \overline{\partial r}\right) \\
& \quad=f^{\prime}(r) \bar{\partial} r \wedge \psi_{p q j} \wedge \bar{\partial} r+f(r) \bar{\partial}\left(\psi_{p q j} \wedge \bar{\partial} r\right) \\
& \quad=0+f(r) \bar{\partial}\left(1 / 2 \bar{z}_{1}^{q-1} z_{n}^{p} d \bar{z}_{1} \wedge \ldots \wedge d \bar{z}_{j} \wedge \bar{\partial}\left(r^{2}\right)\right)=0 .
\end{aligned}
$$

As before, there is a countable sequence $\lambda_{p q 1}^{j 2}, \lambda_{p q 2}^{j 2}, \cdots$ of $\lambda$ 's for which the boundary condition is satisfied, and the corresponding $f_{p q m}^{j 2}(r)=c_{p q j} r^{1-n-p-q}$ 
$\cdot J_{p+q+n-1}\left(\lambda_{p q m}^{j 2} r\right)$ form a complete orthogonal set on $(0,1)$ with respect to the weight function $r$. (The expansion of a function with respect to this basis is a Fourier-Bessel series, but the same theory applies as with the Dini series (cf. Watson [17, Chapter XVIII).) Therefore, letting the unitary group act, we see that $\left\{f_{p q m}^{j 2}(r) \psi_{p q j}^{a} \wedge \bar{\partial} r\right\}$ is an orthonormal basis for the subspace of $\mathbb{C}^{j+1}$ whose elements are in the span of the $\psi \wedge \bar{\partial} r$ 's on each sphere.

Just as we obtained another subspace by applying $\bar{\partial}$ to the $f(r) \phi$ 's, we obtain yet another one by applying $\delta$ to the $f(r) \psi \wedge \overline{\partial r}$ 's, and $\left\{2^{1 / 2}\left(\lambda_{p q m}^{j 2}\right)^{-1} \delta\left(f_{p q m}^{j 2}(r) \psi_{p q j}^{a} \wedge \bar{\partial}_{r}\right)\right\}_{p q m a}$ is an orthonormal basis for this subspace. An explicit formula for these forms will be useful. Since

$$
\begin{aligned}
& D\left(\sum b_{i_{1} \cdots i_{j}} d \bar{z}_{i_{1}} \wedge \cdots \wedge d \bar{z}_{i_{j}}\right) \\
& \quad=2 \sum(-1)^{a}\left(\partial b_{i_{1} \cdots i_{j}} / \partial z_{i_{2}}\right) d \bar{z}_{i_{1}} \wedge \cdots \widehat{d}_{i_{a}} \cdots \wedge d \bar{z}_{i_{j}}
\end{aligned}
$$

we have

$$
\begin{aligned}
& \grave{D}\left(f(r) \psi_{p q j} \wedge \overline{\partial r}\right) \\
& =\sum_{a=j+1}^{n} \sum_{b=1}^{j}(-1)^{b} \frac{\partial}{\partial z_{b}}\left(f(r) \bar{z}_{1}^{q-1} z_{n}^{p} z_{a}\right) d \bar{z}_{1} \wedge \cdots{\widehat{d \bar{z}_{b}}}_{\cdots \wedge} \wedge \bar{z}_{j} \wedge d \bar{z}_{a} \\
& +(-1)^{j+1} \sum_{a=j+1}^{n} \frac{\partial}{\partial z_{a}}\left(f(r) \bar{z}_{1}^{q-1} z_{n}^{p} z_{a}\right) d \bar{z}_{1} \wedge \cdots \wedge d \bar{z}_{j} \\
& =\sum_{a=j+1}^{n} \sum_{b=1}^{j}(-1)^{b} \frac{f^{\prime}(r)}{2 r} \bar{z}_{b} \bar{z}_{1}^{q-1} z_{n}^{p} z_{a} d \bar{z}_{1} \wedge \ldots \widehat{d}_{b} \cdots \wedge d \bar{z}_{j} \wedge d \bar{z}_{a} \\
& +(-1)^{j+1}\left[(p+n-j) f(r) \bar{z}_{1}^{q-1} z_{n}^{p}+\frac{f^{\prime}(r)}{2 r} \bar{z}_{1}^{q-1} z_{n}^{p} \sum_{a=j+1}^{n} z_{a} \bar{z}_{a}\right] d \bar{z}_{1} \wedge \cdots \wedge d \bar{z}_{j} \\
& =\frac{(-1)^{j+1}}{r}\left[\frac{r f^{\prime}(r)}{2}+(p+n-j) f(r)\right] \psi_{p q j}+\frac{2}{r}(p+n-j) f(r) \phi_{p q(j-1)} \wedge \bar{\partial} r .
\end{aligned}
$$

Note that this automatically satisfies the right boundary condition: $f(1)=0$.

We have now obtained the complete decomposition of the spaces $\mathbb{Q}^{j}$. To show this, we need some lemmas.

Lemma 1. If $u=g_{1}(r) \psi_{p q j}+g_{2}(r) \phi_{p q(j-1)} \wedge \overline{\partial r}(p \geq 0)$ then 


$$
\begin{aligned}
& \overline{\partial u}=\left[(-1)^{j} g_{1}^{\prime}(r)+\frac{(q+j-1)}{r} g_{2}(r)+\frac{(-1)^{j}}{r}(2 q+2 j-1) g_{1}(r)\right] \psi_{p q j} \wedge \bar{\partial} r, \\
& \delta u=\left[\frac{(-1)^{j}}{2} g_{2}^{\prime}(r)+\frac{2(p+n-j)}{r} g_{1}(r)+\frac{(-1)^{j}}{2 r}(2 p+2 n-2 j+1) g_{2}(r)\right] \phi_{p q(j-1)^{\circ}}
\end{aligned}
$$

Also, if $u=g(r) \psi_{(-1) q(n-1)}$ then

$$
\bar{\partial} u=\left[(-1)^{n-1} g^{\prime}(r)+\frac{(-1)^{n-1}}{r}(2 q+2 n-3) g(r)\right] \psi_{(-1) q(n-1)} \wedge \bar{\partial} r
$$

and $\delta u=0$.

Proof. Brute force computation.

Lemma 2. The formulas of Lemma 1 are true with $\psi_{p q j}, \phi_{p q(j-1)}$ replaced by $\psi_{p q j}^{a}, \phi_{p q(j-1)}^{a}$.

Proof. The action of the unitary group taking $\phi_{p q(j-1)}$ to $\phi_{p q(j-1)}^{a}$ is the same as the one taking $\psi_{p q j}$ to $\psi_{p q j}^{a}$, by construction of $\psi_{p q j}^{a}$. Since $\frac{1}{\partial}$ and $\delta$ commute with $U(n)$, we are done.

Lemma 3. $\square$ annibilates no form of the type

$$
u=\sum_{p q a}\left(g_{p q 1}^{a}(r) \psi_{p q j}+g_{p q 2}^{a}(r) \phi_{p q(j-1)}^{a} \wedge \bar{\partial} r\right) \quad(p \geq 0) .
$$

Proof. By Schur's lemma it suffices to consider a fixed $(p, q)$, i.e. $u=\Sigma c_{a} u^{a}$ where $u^{a}=g_{1}^{a}(r) \psi_{p q j}^{a}+g_{2}^{a}(r) \phi_{p q(j-1)}^{a} \wedge \bar{\partial} r$. The formula $(\square u, u)=(\bar{\partial} u, \bar{\partial} u)+$ $(\delta u, \delta u)$ shows that $\square u=0$ if and only if $\bar{\partial} u=0$ and $\delta u=0$. Since $\psi_{p q j}^{a}$ (respectively $\phi_{p q(j-1)}^{a}$ ) is orthogonal to $\psi_{p q j}^{a^{\prime}}$ (respectively $\phi_{p q(j-1)}^{a^{\prime}}$ ) for $a \neq a^{\prime}$, Lemma 2 shows that $\bar{\partial} u=0$ and $b u=0$ if and only if $\bar{\partial} u^{a}=0$ and $b u^{a}=0$ for each $a$. Thus it suffices to consider a fixed $a$ and (after a unitary transformation) we may take $a=1$.

Therefore we must show that there is no form $u=g_{1}(r) \psi_{p q j}+g_{2}(r) \phi_{p q(j-1)} \wedge$ $\bar{\partial} r$ which is harmonic and satisfies the $\bar{\partial}$-Neumann conditions. After some computation we find that

$$
\begin{gathered}
u=\bar{z}_{1}^{q-1} z_{n}^{p}\left[\left(r\left\{\frac{p+n-j}{p+q+n-1} g_{1}(r)+\frac{1}{2}(-1)^{j-1} \frac{q+j-1}{p+q+n-1} g_{2}(r)\right\}\right.\right. \\
+\frac{(-1)^{j-1}}{r}\left\{(-1)^{j} g_{1}(r)+\frac{1}{2} g_{2}(r)\right\}
\end{gathered}
$$




$$
\begin{aligned}
\left.\cdot\left\{\frac{p+n-j}{p+q+n-1} \sum_{1}^{j} z_{i} \bar{z}_{i}-\frac{q+j-1}{p+q+n-1} \sum_{j+1}^{n} z_{i} \bar{z}_{i}\right\}\right) d \bar{z}_{1} \wedge \ldots \wedge d \bar{z}_{j} \\
+\frac{1}{r}\left\{(-1)^{j} g_{1}(r)+\frac{1}{2} g_{2}(r)\right\} \\
\left.\sum_{i=1}^{j} \sum_{a=j+1}^{n}(-1)^{i-1} \bar{z}_{i} z_{a} d \bar{z}_{1} \wedge \cdots \widehat{d}_{i} \cdots \wedge d \bar{z}_{j} \wedge d \bar{z}_{a}\right] .
\end{aligned}
$$

The coefficient of each $d \bar{z}_{i_{1}} \wedge \ldots \wedge d \bar{z}_{i_{j}}$ is a sum of homogeneous harmonic polynomials multiplied by functions of $r$. Since $u$ is harmonic if and only if each coefficient is harmonic, and a harmonic function on $B_{n}$ is determined by its values on any sphere about the origin, it follows that these functions of $r$ are constant. Thus

$$
\begin{gathered}
r^{-1}\left[(-1)^{j} g_{1}(r)+1 / 2 g_{2}(r)\right]=c_{1}, \\
r\left[\frac{p+n-j}{p+q+n+1} g_{1}(r)+\frac{1}{2}(-1)^{j-1} \frac{q+j-1}{p+q+n-1} g_{2}(r)\right]=c_{2} .
\end{gathered}
$$

Solving for $g_{1}$ and $g_{2}$, we find

$$
\begin{aligned}
& g_{1}(r)=c_{2} / r+(-1)^{j}[(q+j-1) /(p+q+n-1)] c_{1} r_{0} \\
& g_{2}(r)=2(-1)^{j-1} c_{2} / r+2[(p+n-j) /(p+q+n-1)] c_{1} r .
\end{aligned}
$$

The first boundary condition says $g_{2}(1)=0$, i.e.

$$
(-1)^{j-1} c_{2}+[(p+n-j) /(p+q+n-1)] c_{1}=0 .
$$

By Lemma 1, the second boundary condition says

$$
(-1)^{j} g_{1}^{\prime}(1)+(q+j-1) g_{2}(1)+(-1)^{j}(2 q+2 j-1) g_{1}(1)=0
$$

i.e.

$$
\begin{aligned}
(-1)^{j-1} c_{2}+\frac{q+j-1}{p+q+n-1} c_{1} & +(q+j-1)\left[2(-1)^{j-1} c_{2}+2 \frac{p+n-j}{p+q+n-1} c_{1}\right] \\
& +(-1)^{j}(2 q+2 j-1)\left[c_{2}+(-1)^{j} \frac{q+j-1}{p+q+n-1} c_{1}\right]=0 .
\end{aligned}
$$

The LHS reduces to $[2(p+q+n)(q+j-1) /(p+q+n-1)] c_{1}$. Therefore $c_{1}=0$, whence $c_{2}=0$, which implies $g_{1}=g_{2}=0$. Q.E.D.

Lemma 4. $\square$ annibilates no form of the type $u=\Sigma_{q a} g_{q}^{a}(r) \psi_{(-1) q(n-1)}^{a}$. 
Proof. The same reasoning as in Lemma 3 shows that it suffices to consider

$$
u=g(r) \psi_{(-1) q(n-1)}=\frac{g(r)}{r} \bar{z}_{1}^{q-1} \sum_{i=1}^{n}(-1)^{i+n} \bar{z}_{i} d \bar{z}_{1} \wedge \ldots \widehat{d \bar{z}}_{i} \ldots \wedge d \bar{z}_{n} .
$$

Since $\bar{z}_{1}^{q-1} \bar{z}_{i}$ is harmonic, if $\square u=0$ we would have $g(r) / r=c$. The boundary condition, by Lemma 1 , is $(-1)^{n-1} c+(-1)^{n-1}(2 q+2 n-3) c=0$, i.e. $c=0$. Q.E.D.

Lemma 5. $\square$ is injective on $\mathbb{Q}^{j}$ for $j>0$.

Proof. We have already noted that $\square$ annihilates no forms of the type $g(r) \phi$ or $g(r) \psi \wedge \bar{\partial} r$, and Lemmas 3 and 4 show that $\square$ annihilates no forms in the orthogonal complement.

We are now ready to state the solution of the $\bar{\partial}$-Neumann problem:

Theorem 2.

$$
\begin{aligned}
\left\{f_{p q m}^{j 1}(r) \phi_{p q j}^{a}\right\}_{p q m a} \cup\left\{f_{p q m}^{(j-1) 2}(r) \psi_{p q(j-1)}^{a} \wedge \bar{\partial} r\right\} \\
\cup\left\{2^{1 / 2}\left(\lambda_{p q m}^{(j-1) 1}\right)^{-1} \bar{\partial}\left(f_{p q m}^{(j-1) 1}(r) \phi_{p q(j-1)}^{a}\right)\right\}_{p q m a} \\
\cup\left\{2^{1 / 2}\left(\lambda_{p q m}^{j 2}\right)^{-1} \delta\left(f_{p q m}^{j 2}(r) \psi_{p q j}^{a} \wedge \overline{\partial r}\right)\right\}_{p q m a}
\end{aligned}
$$

is a complete orthonormal basis for $\mathbb{Q}^{j}$ consisting of eigenforms for $2 \square$ with eigenvalues $\left(\lambda_{p q m}^{j 1}\right)^{2},\left(\lambda_{p q m}^{(j-1) 2}\right)^{2},\left(\lambda_{p q m}^{(j-1) 1}\right)^{2},\left(\lambda_{p q m}^{j 2}\right)^{2}$ respectively. (In $\mathbb{Q}^{1}$ (respectively $\mathcal{Q}^{n-1}$ ) forms of the type $\psi \wedge \bar{\partial}$ r (respectively $\phi$ ) do not occur, and in $\mathbb{Q}^{n}$ only forms of the type $\psi \wedge \overline{\partial r}$ occur.)

Proof. We need only show completeness; everything else follows from the foregoing discussion together with the remark that the ranges of $\bar{\partial}$ and $\delta$ are orthogonal.

Let $\mathbb{Q}_{1}^{j}, \mathbb{Q}_{2}^{j}$, and $\mathbb{Q}_{3}^{j}$ denote the spaces of forms of types $\phi, \psi \wedge \bar{\partial} r$, and $g_{1} \psi+g_{2} \phi \wedge \overline{\partial r}$ respectively. Then $\mathbb{Q}_{1}^{j}, \mathbb{Q}_{2}^{j}, \mathbb{Q}_{3}^{j}$ are mutually orthogonal and invariant under $\square$, and $\bigoplus_{1}^{3} \mathbb{Q}_{i}^{j}=\mathbb{Q}^{j}$. We have already seen that $\left\{f_{p q m}^{j 1}(r) \phi_{p q j}^{a}\right\}_{p q m a}$ spans $\mathbb{Q}_{1}^{j}$ and $\left\{f_{p q m}^{(j-1) 2}(r) \psi_{p q j}^{a} \wedge \bar{\partial}_{r}\right\}_{p q m a}$ spans $\mathbb{Q}_{2}^{j}$. It remains to be shown that the other basis elements span $\mathbb{Q}_{3}^{j}$, which amounts to showing that $\bar{\partial}\left(\mathbb{P}_{1}^{j}-1 \cap \operatorname{Dom} \bar{\partial}\right)$ $+\delta\left(\mathbb{Q}_{2}^{j+1} \cap\right.$ Dom $\left.\mathfrak{b}\right)$ is dense in $\mathbb{Q}_{3}^{j}$. Since $\square$ is injective (Lemma 5 ) and selfadjoint, its range is dense. Therefore, given $u \in \mathbb{Q}_{3}^{j}$, we can find $v_{1}, v_{2}, v_{3}, \cdots$ in $\mathbb{Q}_{3}^{j}$ (because $\mathbb{Q}_{3}^{j}$ is invariant) such that $u=\lim \left(\square v_{m}\right)=\lim \left(\bar{\partial} \delta v_{m}+\delta \bar{\partial} v_{m}\right)$. By Lemma 2, $\delta v_{m} \in \mathbb{P}_{1}^{j-1}$ and $\bar{\partial}_{m} \in \mathbb{Q}_{2}^{j+1}$; by construction, $\delta v_{m} \in$ Dom $\bar{\partial}$ and $\bar{\partial} v_{m} \in$ Dom $\delta$, and this is what we needed. Q.E.D.

Corollary. The basis elements of Theorems 1 and 2 (if we take $b_{p q}^{a}=\phi_{p q 0}^{a}$ in Theorem 1) form a canonical basis for $\bigoplus_{0}^{n} \mathbb{Q}^{j}$ in the sense of Kodaira and Spencer [5]. 
The reader may find it reassuring to see the forms $\bar{\partial}\left(f_{p q m}^{j 1}(r) \phi_{p q j}\right)$ and $\mathscr{D}\left(f_{p q m}^{j 2}(r) \psi_{p q j} \wedge \overline{\partial r}\right)$ expressed as $\Sigma a_{i_{1} \ldots i_{j}} d \bar{z}_{i_{1}} \wedge \ldots \wedge d \bar{z}_{i_{j}}$ where the $a_{i_{1}} \ldots i_{j}$ are sums of homogeneous harmonic polynomials multiplied by appropriate Bessel functions, so that it is directly obvious that these forms are eigenvectors for $2 \square$. To accomplish this we use two well-known recursion formulas for Bessel functions, namely

$$
\begin{aligned}
& (d / d x)\left(x^{-\mu} J_{\mu}(x)\right)=-x^{-\mu} J_{\mu+1}(x) \\
& J_{\mu-1}(x)+J_{\mu+1}(x)=(2 \mu / x) J_{\mu}(x) \quad(\text { cf. Watson [17, p. 17]) }
\end{aligned}
$$

Applying these to the expressions for $\bar{\partial}\left(f_{p q m}^{j 1}(r) \phi_{p q j}\right)$ and $\delta\left(f_{p q m}^{j 2}(r) \psi_{p q j} \wedge \overline{\partial r}\right)$ in terms of the $d \bar{z}_{i}$ 's, we obtain (modulo a normalizing constant)

$$
\begin{aligned}
& \bar{\partial}\left(f_{p q m}^{j 1}(r) \phi_{p q j}\right) \\
& =\left(\frac{q+j}{p+q+n-1}\right) \frac{\lambda_{p q m}^{1 j}}{2} r^{2-p-q-n} J_{p+q+n-2}\left(\lambda_{p q m}^{1 j}\right) \bar{z}_{1}^{q-1} z_{n}^{p} d \bar{z}_{1} \wedge \cdots \wedge d \bar{z}_{j+1} \\
& \quad-\frac{\lambda_{p q m}^{1 j}}{2} r^{-n-p-q} J_{p+q+n}\left(\lambda_{p q m}^{1 j} r\right) \bar{z}_{1}^{q-1} z_{n}^{p} \\
& \\
& \quad\left[\left(\frac{p+n-j-1}{p+q+n-1} \sum_{1}^{j+1} z_{i} \bar{z}_{i}-\frac{q+j}{p+q+n-1} \sum_{j+2}^{n} z_{i} \bar{z}_{i}\right) d \bar{z}_{1} \wedge \cdots \wedge d \bar{z}_{j+1}\right. \\
& \left.+\sum_{i=1}^{j+1} \sum_{a=j+2}^{n}(-1)^{i+j-1} \bar{z}_{i} z_{a} d \bar{z}_{1} \wedge \cdots \widehat{d \bar{z}_{i}} \cdots \wedge d \bar{z}_{j} \wedge d \bar{z}_{a}\right]
\end{aligned}
$$

and

$$
\begin{aligned}
\delta & \left(f_{p q m}^{j 2}(r) \psi_{p q j} \wedge \bar{\partial} r\right) \\
= & (-1)^{j+1}\left(\frac{p+n-j}{p+q+n-1}\right) \lambda_{p q m}^{j 2} r^{2-p-q-n} J_{p+q+n-2}\left(\lambda_{p q m}^{j 2} r\right) \bar{z}_{1}^{q-1} z_{n}^{p} d \bar{z}_{1} \wedge \cdots \wedge d \bar{z}_{j} \\
+ & \lambda_{p q m .}^{j 2} r^{-n-p-q} J_{p+q+n}\left(\lambda_{p q m}^{j 2} r\right) \bar{z}_{1}^{q-1} z_{n}^{p} \\
\cdot & {\left[(-1)^{j+1}\left(\frac{p+n-j}{p+q+n-1} \sum_{1}^{j} z_{i} \bar{z}_{i}-\frac{q+j-1}{p+q+n-1} \sum_{j+1}^{n} z_{i} \bar{z}_{i}\right) d \bar{z}_{1} \wedge \cdots \wedge d \bar{z}_{j}\right.} \\
& \left.+\sum_{i=1}^{j} \sum_{a=j+1}^{n}(-1)^{i-1} \bar{z}_{i} z_{a} d \bar{z}_{1} \wedge \cdots \widehat{d}_{i} \cdots \wedge d \bar{z}_{j} \wedge d \bar{z}_{a}\right] .
\end{aligned}
$$


Thus each of these is the sum of a term of harmonic type $(p, q-1)$ and one of harmonic type $(p+1, q)$. Note also that a factor of $\lambda$ appears in each ter $\mathrm{m}$, as we expect from the canonical basis property.

In particular,

$$
\begin{aligned}
& \delta\left(f_{(-1) q m}^{(n-1) 2}(r) \psi_{(-1) q(n-1)} \wedge \overline{\partial r}\right) \\
& =\lambda_{(-1) q m}^{(n-1) 2} r^{-n-q+1} J_{q+n-1}\left(\lambda_{(-1) q m}^{(n-1) 2} r\right) \bar{z}_{1}^{q-1} \sum_{i=1}^{n}(-1)^{i-1} \bar{z}_{i} d \bar{z}_{1} \wedge \ldots \widehat{d \bar{z}}_{i} \ldots \wedge d \bar{z}_{n} \\
& =\lambda_{(-1) q m}^{(n-1) 2} r^{-n-q+1} J_{q+n-1}\left(\lambda_{(-1) q m}^{(n-1) 2} r\right)(-1)^{n-1} r \psi_{(-1) q(n-1)} .
\end{aligned}
$$

Indeed, we could have derived this expansion for the forms $g(r) \psi_{(-1) q(n-1)}$ by observing that the coefficients of $r \psi(-1) q(n-1)$ are harmonic of type $(0, q)$ and using the boundary condition $1 / 2 f^{\prime}(1)+(q+n-1) f(1)=0$ where $f(r)=(1 / r) g(r)$. The recursion formula $x J_{\mu}^{\prime}(x)+\mu J_{\mu}(x)=x J_{\mu-1}(x)$ shows that this boundary condition is equivalent to the boundary condition $f(1)=0$ for $f(r) \psi_{(-1) q(n-1)} \wedge \bar{\partial} r$.

3. Distribution of the eigenvalues. The estimates for the eigenvalues of are obtained from facts about the zeros of Bessel functions (Watson [17, Chapter $\mathrm{XVl)}$. We summarize the ma in results in a theorem:

Theorem 3. (1) For some constant $c=c(p, q, j), \lambda_{p q m}^{j 1}=c+m \pi+\Theta(1 / m)$, and for some constant $c=c(p+q), \lambda_{p q m}^{j 2}=c+m \pi+\mathcal{O}(1 / m)$.

(2) Let $\nu=p+q+n-1, H=q-p+2 j-n+1$. Then

$$
\begin{aligned}
(\nu(\nu+2))^{1 / 2} & <\lambda_{p q 1}^{j 2}<(2(\nu+1)(\nu+2))^{1 / 2}, \\
(\nu(\nu+2))^{1 / 2} & <\lambda_{p q 1}^{j 1}<(2(\nu+1)(\nu+3))^{1 / 2} \text { if } H \geq 0, \\
((\nu+2)(\nu+H))^{1 / 2} & <\lambda_{p q 1}^{j 1}<(2(\nu+1)(\nu+H))^{1 / 2} \quad \text { if }-\nu<H<0, \\
((\nu+1)(\nu+3))^{1 / 2} & <\lambda_{p 01}^{01}<(2(\nu+2)(\nu+4))^{1 / 2} \quad(\text { for which } H=-\nu) .
\end{aligned}
$$

Proof. With $\nu, H$ as above, $\lambda_{p q m}^{j 1}$ is the $m$ th positive zero of $\lambda J_{\nu}^{\prime}(\lambda)+H J_{\nu}(\lambda)$, and $\lambda_{p q m}^{j 2}$ is the $m$ th positive zero of $J_{\nu}(\lambda)$. The asymptotic formulas of (1) for these zeros were derived by C. N. Moore [12].

Denote by $j_{\mu}\left(j_{\mu}^{\prime}\right)$ the smallest positive zero of $J_{\mu}\left(J_{\mu}^{\prime}\right)$. Then $\lambda_{p q 1}^{j 2}=j_{\nu}$, and since $J_{\nu}(\lambda), J_{\mu}^{\prime}(\lambda)$ are positive for small $\lambda$ and their zeros are intertwined, we have $j_{\nu}^{\prime}<\lambda_{p q 1}^{j 1}<j_{\nu}$ provided $H \geq 0$. Also, for $H=-\nu$, the recursion formula $\lambda J_{\nu}^{\prime}(\lambda)-\nu J_{\nu}(\lambda)=-\lambda J_{\nu+1}(\lambda)$ shows that $\lambda_{p 01}^{01}=j_{\nu+1}$. The estimates for $\lambda_{p q 1}^{j 2}, \lambda_{p q 1}^{j 1}$ $(H \geq 0)$, and $\lambda_{p 01}^{01}$ then follow from the estimates for $j_{\mu}, j_{\mu}^{\prime}$ in Watson $[17$, p. 486]. 
For the case $-\nu<H<0$, we use higher recursion formulas. First

$$
\lambda J_{\nu}^{\prime}(\lambda)+H J_{\nu}(\lambda)=(\nu+H) J_{\nu}(\lambda)-\lambda J_{\nu+1}(\lambda) .
$$

Since $\nu+H>0$ and $J_{\mu}(\lambda)=\lambda^{\mu}\left[1+\mathcal{O}\left(\lambda^{2}\right)\right]$, it follows that $\lambda J_{\nu}^{\prime}(\lambda)+H J_{\nu}(\lambda)>0$ for small $\lambda$, whence $\lambda_{p q 1}^{j 1}<j_{\nu}^{\prime}$. Next,

$$
2(\nu+1)\left[\lambda J_{\nu}^{\prime}(\lambda)+H J_{\nu}(\lambda)\right]=-\lambda^{2} J_{\nu+2}(\lambda)+\left[2(\nu+1)(\nu+H)-\lambda^{2}\right] J_{\nu}(\lambda) .
$$

Setting $\lambda=\lambda_{p q 1}^{j 1}$, the LHS vanishes; however, since $\lambda_{p q 1}^{j 1}<j_{\nu}^{\prime}<j_{\nu}<j_{\nu+2}$, it follows that $J_{\nu}\left(\lambda_{p q 1}^{j 1}\right)>0, J_{\nu_{+} 2}\left(\lambda_{p q 1}^{j 1}\right)>0$. Therefore we must have $\lambda_{p q 1}^{j 1}<$ $(2(\nu+1)(\nu+H)) \frac{1}{2}$. Finally,

$$
\begin{aligned}
\lambda J_{\nu+2}^{\prime}(\lambda)= & {\left[2(\nu+1)+H-2(\nu+1)(\nu+2)(\nu+H) / \lambda^{2}\right] J_{\nu}(\lambda) } \\
& -\left[1-2(\nu+1)(\nu+2) / \lambda^{2}\right]\left[\lambda J_{\nu}^{\prime}(\lambda)+H J_{\nu}(\lambda)\right] .
\end{aligned}
$$

Setting $\lambda=\lambda_{p q 1}^{j 1}$, the second term on the RHS vanishes. If $\lambda_{p q 1}^{j 1} \leq((\nu+2)(\nu+H))^{1 / 2}$, the RHS would be $\leq H J_{\nu}\left(\lambda_{p q 1}^{j 1}\right)<0$; but $((\nu+2)(\nu+H))^{1 / 2}<\nu+2<j_{\nu+2}^{\prime}$, so the LHS would be positive; contradiction. Q.E.D.

Corollary. $\square$ bas closed range; and if $j>0, \square$ is surjective on $\mathbb{Q}^{j}$. Moreover, the Neumann operator $N$ (defined by $N=0$ on the null space of $\square$ and $N=\square^{-1}$ on the orthogonal complement) is compact.

Proof. (2) implies that all nonzero eigenvalues of $2 \square$ are $>n$, so the restriction of $\square$ to the orthogonal complement of the null space is bounded below and hence has closed range. $\square$ is therefore surjective for $j>0$ by Lemma $5, \S 2$. Moreover, (2) implies that the inequalities $\lambda_{p q m}^{j 1}<c$ or $\lambda_{p q m}^{j 2}<c$ can hold only when $(2(p+q))^{1 / 2}<c$ (the worst case being $\left.\lambda_{p q 1}^{j 1}\right)$, and (1) then implies that there are only finitely many eigenvalues less than any fixed $c$. The eigenvalues of $N$ being the reciprocals of the eigenvalues of $\square$, the spectrum of $N$ is discrete with only zero as a limit point. Since each $\lambda_{p q m}^{j 1}, \lambda_{p q m}^{j 2}$ is the eigenvalue for a finite-dimensional space (namely an irreducible representation space of $U(n)$ ), it follows that $N$ is the norm-limit of operators of finite rank and hence compact. Q.E.D.

4. The $\bar{\partial}$-Neumann problem on an annulus. The theory of $\$ 1$ and $\$ 2$ can be easily adapted to give the solution of the $\bar{\partial}$-Neumann problem on the annulus $A_{\rho}$ $=\left\{z \in \mathbf{C}^{n}: \rho \leq|z| \leq 1\right\}, 0<\rho<1$. The functions $f(r)$ will now be general cylinder functions,

$$
f(r)=r^{1-n-p-q}\left[c_{1} J_{p+q+n-1}(\lambda r)+c_{2} Y_{p+q+n-1}(\lambda r)\right],
$$

and we will have to impose an additional boundary condition at $r=\rho$ :

$$
\rho f^{\prime}(\rho) / 2+(q+j) f(\rho)=0
$$


for forms of type $\phi$ and $f(\rho)=0$ for form of type $\psi \wedge \overline{\partial r}$. The operator

$$
d^{2} / d r^{2}+[(2 n+2 p+2 q-1) / r] d / d r
$$

is nonsingular on the interval $[\rho, 1]$, so the ordinary Sturm-Liouville theory (cf. Coddington and Levinson [2, Chapter 7]) guarantees the existence of complete eigenfunction expansions for the $f(r)^{\prime}$ 's. Thus we obtain numbers $\lambda_{p q m}^{j 1}(\rho)$, $\lambda_{p q m}^{j 2}(\rho)$ and corresponding functions $f_{p q m}^{j 1}(\rho ; r), f_{p q m}^{j 2}(\rho ; r)$ which yield a complete orthogonal decomposition of the spaces $\mathcal{Q}^{j}\left(A_{\rho}\right)$ just as in Theorems 1 and 2.

The main difference is in the harmonic spaces. If $b_{p q}$ is a harmonic polynomial of type $(p, q)$, then $b_{p q}$ and $r^{2(1-n-p-q)} b_{p q}$ are both harmonic on $A_{\rho}$, so for $\lambda=0$ we must take $f(r)=c_{1}+c_{2} r^{2(1-n-p-q)}$. It is easy to see that no such $f(r)$ can satisfy the boundary conditions

$$
r f^{\prime}(r) / 2+(q+j) f(r)=0 \quad(j \leq n-2)
$$

or $f(r)=0$ at both $r=1$ and $r=\rho$, except for the case $q=j=0$ when $f(r)=c_{1}$ works; thus there are no harmonic forms of the types $\phi$ or $\psi \wedge \overline{\partial r}$ except for the harmonic space of functions which we have already found for $\rho=0$. Lemma 3 of $\$ 2$ also remains valid, although the linear algebra in the proof becomes rather more formidable. Lemma 4, however, breaks down: $g(r)=r^{3-2 n-2 q}$ satisfies the boundary conditions given by Lemma 1 , so we obtain an infinite-dimensional harmonic space in $\mathbb{Q}^{n-1}\left(A_{\rho}\right)$ spanned by $\left\{r^{3-2 n-2 q} \psi_{(-1) q(n-1)}^{a}\right\}_{q a}$. This comes as no surprise, considering the behavior of the $\bar{\partial}_{b}$ complex. It is also indicated by the general theory of the $\bar{\partial}$-Neumann problem since the basic estimate of Kohn [6] for $A_{\rho}$ fails to hold for $j=0$ and $j=n-1$.

We do not have precise results about the eigenvalues corresponding to Theorem 3 , since the equations defining the $\lambda(\rho)$ 's are considerably more complicated. In fact, in the notation of Theorem 3, if $F_{1}(\lambda, r)=\lambda r J_{\nu}^{\prime}(\lambda r)+H J_{\nu}(\lambda r)$ (respectively $\left.F_{1}(\lambda, r)=J_{\nu}(\lambda r)\right)$ and $F_{2}(\lambda, r)=\lambda r Y_{\nu}^{\prime}(\lambda r)+H Y_{\nu}(\lambda r)$ (respectively $F_{2}(\lambda, r)=$ $Y_{\nu}(\lambda r)$ ) then the equation for $\lambda_{p q m}^{j 1}(\rho)$ (respectively $\lambda_{p q m}^{j 2}(\rho)$ ) is

$$
F_{1}(\lambda, 1) F_{2}(\lambda, \rho)-F_{1}(\lambda, \rho) F_{2}(\lambda, 1)=0 .
$$

We can state the following proposition:

Theorem 4. The functions $\lambda_{p q m}^{j 1}(\rho)$ and $\lambda_{p q m}^{j 2}(\rho)$ are continuous on the balfopen interval $[0,1)$.

Proof. Continuity at 0 follows from Theorem 3.1 of Coddington and Levinson [2, Chapter 91, since the operator

$$
d^{2} / d r^{2}+[(2 n+2 p+2 q-1) / r] d / d r
$$

is of limit-point type at 0 . Continuity on $(0,1)$ follows from Theorem 4.1 (ibid.), 
as this theorem works equally well when the equation is nonsingular at the endpoints. Q.E.D.

Unfortunately, to obtain the qualitative results of $\$ 3$ a stronger result such as equicontinuity of the $\lambda(\rho)$ 's is necessary. However, indications are quite strong that the qualitative behavior of the $\bar{\partial}$-Neumann problem for $\rho>0$ is the same as for $\rho=0$ except for the existence of the extra harmonic space.

5. Sobolev estimates for $\square$. We now return to the complete unit ball. From the general theory of the $\cdot \bar{\partial}$-Neumann problem it is known that $\|u\|_{s+1} \leqslant\|\square u\|_{s} \leqslant$ $\|u\|_{s+2}$ for $u \in \mathbb{Q}^{j} \cap \operatorname{Dom}(\square), j>0$. Our investigation of the $\bar{\partial}_{b}$ complex, in which the Laplacian $\square_{b}$ satisfied the same estimate, showed that the strength of $\square_{b}$ on $u$ depends on the spherical harmonic decomposition of $u: \square_{b}$ is strongest on forms with $p-q=$ const. and weakest on forms with $p=$ const. or $q$ = const. We are therefore led to expect that $\square$ may exhibit a similar behavior, except that it is likely to be less symmetric in $p$ and $q$. Indeed, this seems to be the case.

The search for a precise relationship betwe en $\square$ and the Sobolev norms is unfortunately beset by a series of technical difficulties which will become apparent, so our results are rather incomplete. However, we are able to shed some light on the most essential features of the situation. We can display infinitedimensional subspaces of $\mathbb{Q}^{j}(0 \leq j \leq n-1)$ on which $\|\square u\| \leqslant\|u\|_{1}$ (whence $\|\square u\| \sim\|u\|_{1}$ for $j \geq 1$ by general theory), and these spaces occur with $q=$ const. (On $\mathbb{Q}^{n}$ the $\bar{\partial}$-Neumann conditions coincide with the Dirichlet conditions, which are coercive; cf. Dunford and Schwartz [3, §XIV.6]. Hence $\|\square u\| \sim\|u\|_{2}$ on $\mathbb{Q}^{n}$.) Moreover, we show that $\|\square u\| \sim\|u\|_{2}$ on the subspace of $\mathbb{Q}^{j}$ with $p=$ $q+2 j$.

First we consider functions, where we naturally restrict our attention to the orthogonal complement of the harmonic space. The technique is to compare the $\bar{\partial}$-Neumann problem with the coercive $d$-Neumann problem. Thus let $\Delta$ denote the selfadjoint extension of the Laplace-de Rham operator $-4 \Sigma\left(\partial^{2} / \partial z_{i} \partial \bar{z}_{i}\right)$ determined by the boundary condition $\left.\langle d u, d r\rangle\right|_{r=1}=0$. For $u \in \operatorname{Dom}(\Delta)$ we have

$$
\left(\Delta^{1 / 2} u, \Delta^{1 / 2} u\right)=(\Delta u, u)=(d u, d u) \sim\|u\|_{1}
$$

(provided $u$ is orthogonal to the constants), so by continuity $\left\|\Delta^{1 / 2} u\right\| \sim\|u\|_{1}$ for $u \in \operatorname{Dom}\left(\Delta^{1 / 2}\right)$. More generally one can show that $\operatorname{Dom}\left(\Delta^{s / 2}\right)$ is a closed subspace of $H_{s}$, and $\Delta^{s / 2}$ : $\operatorname{Dom}\left(\Delta^{s / 2}\right) \subset H_{s} \rightarrow L^{2}$ is a topological isomorphism. However, we will see from the proof of Lemma 2 that, for $s \geq 3 / 2, H_{s} \cap \operatorname{Dom}(\square)$ $\not \subset \operatorname{Dom}\left(\Delta^{s / 2}\right)$, so this method will not yield higher $s$-norm estimates for $\square$.

The eigenfunction decomposition of $\mathbb{Q}^{0}$ for $\Delta$ proceeds just as in $\$ 1$. The $d$-Neumann problem does not respect the complex structure, so only the total degree $k=p+q$ of the spherical harmonics is relevant. If we look for a solution of 
$\Delta-\mu^{2}=0$ of the form $f(r) b_{k}$ where $b_{k}$ is a harmonic polynomial of degree $k$, we get the modified Bessel equation as before and hence $f(r)=c r^{1-n-k} J_{n+k-1}(\mu r)$. The boundary condition is $f^{\prime}(1)+k f(1)=0$; equivalently $\mu J_{n+k-1}^{\prime}(\mu)+$ $(1-n) J_{n+k-1}(\mu)=0$. We denote the positive roots of this equation by $\mu_{k 1}, \mu_{k 2}, \cdots$, and the corresponding $f$ 's (normalized as usual) by $f_{k 1}, f_{k 2}, \ldots$. Theorem 3 gives the behavior of the $\mu_{k l}$ 's, taking $H=1-n$.

The first thing we notice is that for $p=q$ the $\bar{\partial}$-Neumann and $d$-Neumann conditions coincide. Hence

Lemma 1. If $u$ is in the span of the $f_{p p m}^{01} b_{p p}$ 's, $\|2 \square u\|=\|\Delta u\| \sim\|u\|_{2}$.

The next step is to compute the change-of-basis matrix from the $\square$-eigenbasis to the $\Delta$-eigenbasis. For brevity we shall write $\lambda_{p q m}^{01}=\lambda_{p q m}, f_{p q m}^{01}=f_{p q m}$, $\nu=p+q+n-1, H=q-p-n+1, K=1-n$. Then if $b_{p q}$ is a harmonic polynomial of type $(p, q)$ with $\int_{S_{n}}\left|b_{p q}\right|^{2}=1$, we have

$$
\begin{aligned}
A_{p q m l} & \equiv\left(f_{p q m}(r) b_{p q}, f_{k l}(r) b_{p q}\right) \\
& =\frac{\int_{0}^{1} J_{\nu}\left(\lambda_{p q m} r\right) J_{\nu}\left(\mu_{k l} r\right) r d r}{\left[\int_{0}^{1} J_{\nu}\left(\lambda_{p q m} r\right)^{2} r d r \int_{0}^{1} J_{\nu}\left(\mu_{k l} r\right)^{2} r d r\right]^{1 / 2}} .
\end{aligned}
$$

Using formulas from Watson [17, pp. 134-135] together with the boundary conditions, we find

$$
\begin{aligned}
& \int_{0}^{1} J_{\nu}\left(\lambda_{p q m} r\right)^{\prime} J_{\nu}\left(\mu_{k l} r\right) r d r=\frac{(q-p) J_{\nu}\left(\lambda_{p q m}\right) J_{\nu}\left(\mu_{k l}\right)}{\lambda_{p q m}^{2}-\mu_{k l}^{2}} \quad(q \neq p), \\
& \int_{0}^{1} J_{\nu}\left(\lambda_{p q m}\right)^{2} r d r=\frac{1}{2} J_{\nu}\left(\lambda_{p q m}\right)^{2}\left[1+\frac{H^{2}-\nu^{2}}{\lambda_{p q m}^{2}}\right], \\
& \int_{0}^{1} J_{\nu}\left(\mu_{k l} r\right)^{2} r d r=\frac{1}{2} J_{\nu}\left(\mu_{k l}\right)^{2}\left[1+\frac{K^{2}-\nu^{2}}{\mu_{k l}^{2}}\right],
\end{aligned}
$$

and so

$$
\begin{aligned}
\left|A_{p q m l}\right| & =\frac{2|q-p|}{\left|\lambda_{p q m}^{2}-\mu_{k l}^{2}\right|\left[1+\left(H^{2}-\nu^{2}\right) / \lambda_{p q m}^{2}\right]^{1 / 2}\left[1+\left(K^{2}-\nu^{2}\right) / \mu_{k l}^{2}\right]^{1 / 2}} \quad(q \neq p), \\
A_{p p m l} & =\delta_{m l}
\end{aligned}
$$

Lemma 2. $f_{p q m}(r) b_{p q} \in \operatorname{Dom}\left(\Delta^{1 / 2}\right)$.

Proof. We have 
so

$$
f_{p q m}(r) b_{p q}=\sum_{l=1}^{\infty} A_{p q m l} f_{k l}(r) b_{p q}
$$

$$
\Delta^{1 / 2}\left(f_{p q m}(r) b_{p q}\right)=\sum_{l=1}^{\infty} \mu_{k l} A_{p q m l} f_{k l}(r) b_{p q},
$$

and it suffices to show that the latter sum converges in $L^{2}$, i.e.

$$
\sum_{l=1}^{\infty} \mu_{k l}^{2}\left|A_{p q m l}\right|^{2}<\infty \text {. }
$$

Since $K^{2}<\nu^{2}$, the term $1+\left(K^{2}-\nu^{2}\right) / \mu_{k l}^{2}$ is less than one and increases as $l \rightarrow \infty$. Therefore

$$
\begin{aligned}
& \sum_{l=1}^{\infty} \mu_{k l}^{2}\left|A_{p q m l}\right|^{2} \\
& \quad \leq 2|q-p|\left[1+\frac{H^{2}-\nu^{2}}{\lambda_{p q m}^{2}}\right]^{-1}\left[1+\frac{K^{2}-\nu^{2}}{\mu_{k 1}^{2}}\right]^{-1} \sum_{k=1}^{\infty} \frac{\mu_{k l}^{2}}{\left|\lambda_{p q m}^{2}-\mu_{k l}^{2}\right|^{2}} .
\end{aligned}
$$

But $\mu_{k l}=c_{k}+l \pi+\mathcal{O}(1 / l)$ by Theorem 3, so for sufficiently large $l$, $\mu_{k l}^{2} /\left|\lambda_{p q m}^{2}-\mu_{k l}^{2}\right|^{2} \leq(2 l \pi)^{2} /(1 / 2 l \pi)^{4}$, and thus

$$
\sum_{l=1}^{\infty} \frac{\mu_{k l}^{2}}{\left|\lambda_{p q m}^{2}-\mu_{k l}^{2}\right|^{2}} \leq c \sum_{l=1}^{\infty} \frac{1}{(l \pi)^{2}}<\infty . \quad \text { Q.E.D. }
$$

The following lemma provides the crucial step:

Lemma 3. If $u$ is in the span of $\left\{f_{p q 1}(r) b_{p q}: 0 \leq p<\infty, q=\right.$ const. $\left.\neq 0\right\}$, then $\|\square u\| \lesssim\|u\|_{1}$.

Proof. By Lemma 2, it suffices to show that $\left\|2 \square\left(f_{p q 1}(r) b_{p q}\right)\right\| \leq$ $c\left\|\Delta^{1 / 2}\left(f_{p q 1}(r) b_{p q}\right)\right\|$ with $c$ independent of $p$. Well, $\Sigma_{l=1}^{\infty}\left|A_{p q 1}\right|^{2}=1$ by the Parseval theorem, so

$$
\begin{aligned}
\| 2 & \square\left(f_{p q 1}(r) b_{p q}\right) \|^{2} \\
& =\lambda_{p q 1}^{4}=\lambda_{p q 1}^{4} \sum_{l=1}^{\infty}\left|A_{p q 1 l}\right|^{2}=\frac{\lambda_{p q 1}^{4}}{\mu_{k 1}^{2}} \sum_{l=1}^{\infty} \mu_{k 1}^{2}\left|A_{p q 1 l}\right|^{2} \\
& \leq \frac{\lambda_{p q 1}^{4}}{\mu_{k 1}^{2}} \sum_{l=1}^{\infty} \mu_{k l}^{2}\left|A_{p q 1 l}\right|^{2}=\frac{\lambda_{p q 1}^{4}}{\mu_{k 1}^{2}}\left\|\Delta^{1 / 2}\left(f_{p q 1}(r) b_{p q}\right)\right\|^{2}
\end{aligned}
$$


But by Theorem 3,

$$
\lambda_{p q 1}^{4}<[2(\nu+H)(\nu+1)]^{2}=[4 q(p+q+n)]^{2}
$$

for $p$ sufficiently large so that $H<0$, and

$$
\mu_{k 1}^{2}>(\nu+K)(\nu+2)=(p+q)(p+q+n+1) .
$$

From this it is clear that $\lambda_{p q 1}^{4} / \mu_{k 1}^{2} \leq$ const. as $p \rightarrow \infty$. Q.E.D.

It is essential to take $m=1$ in this lemma. One can easily see that $\lambda_{p q 1}<$ $\mu_{k 1}<\lambda_{p q 2}<\mu_{k 2}<\cdots$, so that all the $\lambda_{p q m^{\prime}} m>1$, are reasonably large. The bad behavior is caused by the smallness of the first eigenvalue when $q-p \ll 0$. (When $q=0$, of course, the first eigenvalue is actually zero.)

A more thorough investigation of the coefficients $A_{p q m l}$ should yield more precise estimates for $\square$. However, this seems to depend on some rather delicate and abstruse estimates for $\lambda_{p q m}$ and $\mu_{k l}$, which are beyond the range of the present author's expertise.

We now apply the above results to forms of the type $f(r) \phi_{p q j}^{a}$ with $j>0$. Each coefficient in $\phi_{p q j}^{a}$ is a homogeneous harmonic polynomial of type $(p, q)$. Since we may define the 1-norm of a form to be the square root of the sum of the squares of the 1-norms of its coefficients, Lemma 2 provides the method for calculating the 1 -norm of $f(r) \phi_{p q j^{*}}^{a}$. The numbers $\lambda_{p q 1}^{j 1}$ satisfy the same estimates as $\lambda_{p q 1}^{01}$ (with $\nu+H=2 q+2 j$ ); therefore Lemma 3 has an exact analogue: $\left\|\square\left(f_{p q 1}^{j 1}(r) \phi_{p q j}^{a}\right)\right\| \leq c\left\|f_{p q 1}^{j 1}(r) \phi_{p q j}^{a}\right\|_{1}$ with $c$ independent of $p$ when $q=$ const. We have

Theorem 5. Suppose $u \in \mathbb{Q}^{j} \cap \operatorname{Dom}\left(\square, j>0\right.$. Then $\|\square u\| \sim\|u\|_{1}$ when $u$ is in the span of $\left\{f_{p q 1}^{j 1}(r) \phi_{p q j}^{a}\right\}_{p a}(q=$ const. $)$, and $\|\square u\| \sim\|u\|_{2}$ when $u$ is in the span of $\left\{f_{(q+2 j) q m}^{j 1} \phi_{(q+2 j) q j}^{a}\right\}_{q m a}$ or in the span of $\left\{f_{p q m}^{j 2}(r) \psi_{p q(j-1)}^{a} \wedge \overline{\partial r}_{p q m a}\right.$.

Proof. The first assertion follows from the preceding observations and the fact that $\|u\|_{1} \lesssim\|\square u\|$ for $j>0$. The $\bar{\partial}$-Neumann conditions for forms of the type $f_{p q m}^{j 1}(r) \phi_{p q j}^{a}$ with $p=q+2 j$ coincide with the $d$-Neumann conditions for their coefficients, and for forms of the type $f_{p q m}^{j 2}(r) \psi_{p q(j-1)}^{a} \wedge \overline{\partial r}$ they coincide with the Dirichlet conditions for the ir coefficients. Since, as we have remarked, both of these are coercive, the theorem is proved. Q.E.D.

It should be true more generally that $\|\square u\| \sim\|u\|_{2}$ for $u$ of the type $f(r) \phi_{p q j}^{a}, p-q=$ const. A possible approach would be to show that the boundary conditions $f^{\prime}(1)+(p+q+c) f(1)=0$ for functions of the type $f(r) b_{p q}$, where $c$ is independent of $p$ and $q$, define a coercive problem, but we have not worked out the details. It is also probable that the estimates which hold for forms of the type $f(r) \phi$ and $f(r) \psi \wedge \bar{\partial} r$ also hold for those of the types $\bar{\partial}(f(r) \phi)$ and $\delta(f(r) \phi \wedge \overline{\partial r})$, respectively. However, the latter forms are not directly amenable to our 
methods, since their coefficients are not homogeneous harmonic polynomials. We therefore leave these assertions in the realm of conjecture.

\section{REFERENCES} 1970.

1. H. Boerner, Representations of groups, 2nd ed., American Elsevier, New York,

2. E. A. Coddington and N. Levinson, Theory of ordinary differential equations, McGraw-Hill, New York, 1955. MR 16, 1022.

3. N. Dunford and J. T. Schwartz, Linear operators. II: Spectral theory. Selfadjoint operators in Hilbert space, Interscience, New York, 1963. MR 32 \#6181.

4. F. Hirzebruch, Topological methods in algebraic geometry, 3rd ed., Die Grundlehren der math. Wissenschaften, Band 131, Springer-Verlag, New York, 1966. MR 34 \#2573.

5. K. Kodaira and D. C. Spencer, On deformations of complex analytic structures. III. Stability theorems for complex structures, Ann. of Math. (2) 71 (1960), 43-76. MR 22 \#5991.

6. J. J. Kohn, Harmonic integrals on strongly pseudo-convex manifolds, I, II, Ann. of Math. (2) 78 (1963), 112-148; ibid. 79 (1964), 450-472. MR 27 \#2999; MR 34 \#8010.

7. - Boundaries of complex manifolds, Proc. Conference Complex Analysis (Minneapolis, 1964), Springer, Berlin, 1965, pp. 81-94. MR 30 \#5334.

8. J. J. Kohn and L. Nirenberg, Non-coercive boundary value problems, Comm. Pure Appl. Math. 18 (1965), 443-492. MR 31 \#6041.

9. J. J. Kohn and H. Rossi, On the extension of holomorphic functions from the boundary of a complex manifold, Ann. of Math. (2) 81 (1965), 451-472. MR 31 \#1399.

10. H. Lewy, On the local character of the solutions of an atypical linear differential equation in three variables and a related theorem for regular functions of two complex variables, Ann. of Math. (2) 64 (1956), 514-522. MR 18, 473.

11. - An example of a smooth linear partial differential equation without solution, Ann. of Math. (2) 66 (1957), 155-158. MR 19, 551.

12. C. N. Moore, The summability of the developments in Bessel functions, with applications, Trans. Amer. Math. Soc. 10 (1909), 391-435.

13. C. Müller, Spherical harmonics, Lecture Notes in Math., no. 17, Springer-Verlag, New York, 1966. MR 33 \#7593.

14. E. M. Stein, Topics in harmonic analysis related to the Littlewood-Paley theory, Ann. of Math. Studies, no. 63, Princeton Univ. Press, Princeton, N. J.; Univ. of Tokyo Press, Tokyo, 1970. MR $40 \# 6176$.

15. W. J. Sweeney, The D-Neumann problem, Acta Math. 120 (1968), 233-277.

MR $37 \# 2250$.

16. F. Trèves, On local solvability of linear partial differential equations, Bull. Amer. Math. Soc. 76 (1970), 552-571. MR 41 \#2200.

17. G. N. Watson, A treatise on the theory of Bessel functions, Cambridge Univ. Press Press, Cambridge,; Macmillan, New York, 1944. MR 6, 64.

18. A. Weil, L'intégration dans les groupes topologiques et ses applications, Actualités Sci. Indust., no. 869, Hermann, Paris, 1940. MR 3, 198. 1946.

19. H. Weyl, The classical groups, 2nd ed., Princeton Univ. Press, Princeton, N. J.,

COURANT INSTITUTE OF MATHEMATICAL SCIENCES, NEW YORK UNIVERSITY, NEW YORK, NEW YORK 10012 